\title{
NBSIR 87-3519
}

\section{6-87 Directory of NVLAP Accredited Laboratories}

Harvey W. Berger, Editor

U.S. DEPARTMENT OF COMMERCE

National Bureau of Standards

Office of Product Standards Policy

Gaithersburg, MD 20899

January 1987

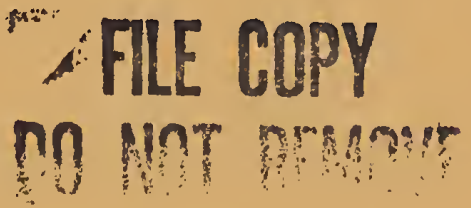



NBSIR $87-3519$

1986-87 DIRECTORY OF NVLAP ACCREDITED LABORATORIES

Harvey W. Berger, Editor

U.S. DEPARTMENT OF COMMERCE

National Bureau of Standards

Office of Product Standards Policy

Gaithersburg, MD 20899

January 1987

U.S. DEPARTMENT OF COMMERCE, Malcolm Baldrige, Secretary NATIONAL BUREAU OF STANDARDS, Ernest Ambler, Director 
The National Bureau of Standards' National Voluntary Laboratory Accreditation Program (NVAP) improves the competence of testing laboratories and the reliability of laboratory measurements. Laboratory ability to meet NVAP criteria and technical requirements, for accreditation of specific test methods, is determined through on-site assessments by technical experts, and laboratory participation in proficiency testing programs. Publication of results of proficiency testing and participation in standards development contribute to improved test methods and laboratory per formance.

The accredited laboratories have been found competent to perform the specific test methods shown. They have the skilled people, necessary facilities and equipment, and documentation and quality assurance systems to produce reliable test data. We recommend that consideration be given to the use of these laboratories whenever their accredited testing capabilities satisfy testing needs.

NVAP has pursued its activities to accredit laboratories in testing areas for which there has been a specific request, a demonstrated need, and a potential benefit to the public. NVLAP has also provided the basis for acceptance by other countries of test data produced by laboratories in the United States through bilateral agreements. We shall continue to work toward liberalizing the means to satisfying trade requirements whenever possible.

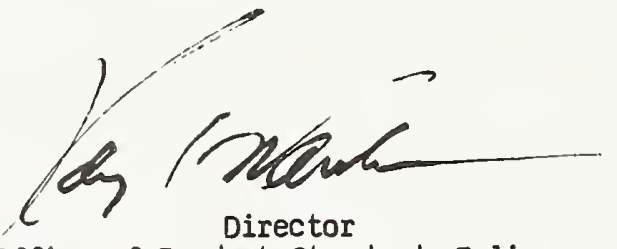

office of Product Standards Policy 

DIRECTORY OF NLAP ACCREDITED LABORATORIES

Contents

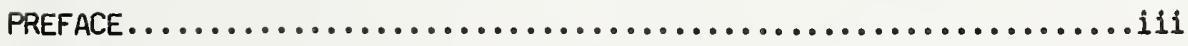

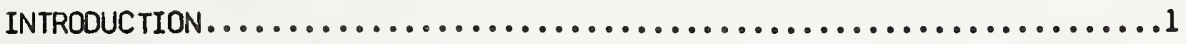

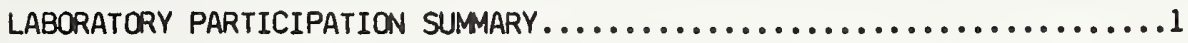

INDEXES

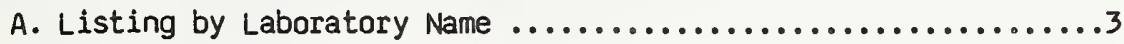

B. Listing by Field of Testing $\ldots \ldots \ldots \ldots \ldots \ldots \ldots \ldots \ldots \ldots$

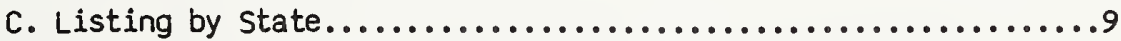

D. Sumnary of Accredited Laboratories by Test Methods and Field of Testing........................................13

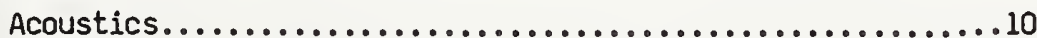

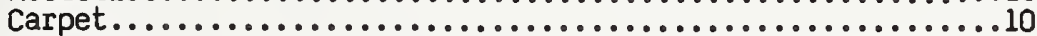

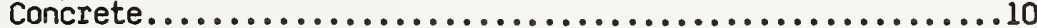

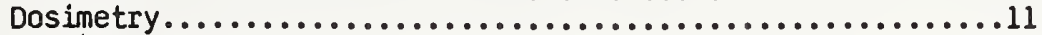

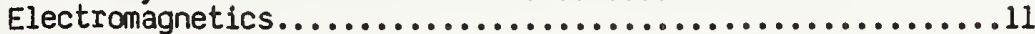

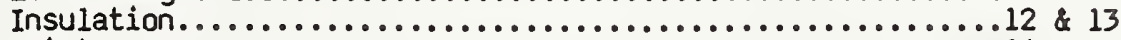

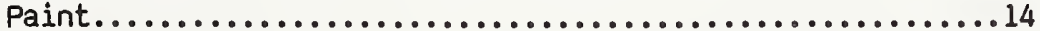

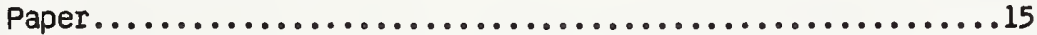

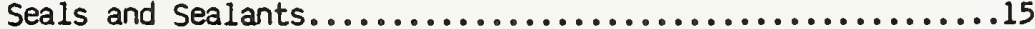

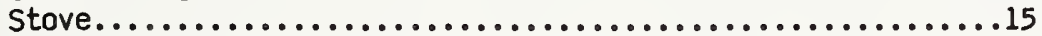

E. Accredited Laboratories and Test Methods for which they are accredited

Lab Code 0101-0125..............................17-27

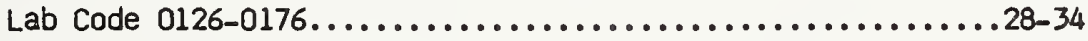

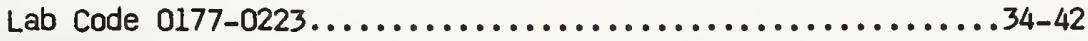

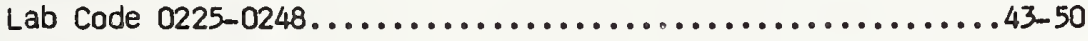

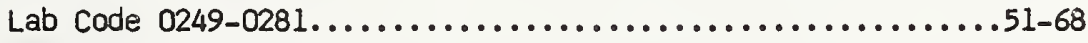

Lab Code 0501-0547..............................69-83

NRLAP PROCEDURES....................................... 84 



\title{
Introduction
}

This Directory lists laboratories accredited, under the procedures of the National Voluntary Laboratory Accreditation Program (NVLAP), as of January 1,1987 . Indexes A, B, and $C$ list the accredited laboratories alphabetically by name, by field of testing, and by state, respectively. Each laboratory's scope of accreditation, which lists the specific test methods for which it is accredited, is presented in Index E.

The period of accreditation is generally one year. Since a laboratory can be accredited on January 1 , April 1 , July 1 , or October 1 , its accreditation will terminate, and must be renewed by that date to remain in force. Users of this Directory, considering the use of accredited laboratories, should be cognizant of the accreditation renewal date and determine if the laboratory is accredited at the time its services are to be provided. Current information on the accredited status of a laboratory can be obtained from the laboratory or by writing to the following address or calling NVLAP on 301-975-4016.

\author{
Manager, Laboratory Accreditation \\ National Bureau of Standards \\ Admin A531 \\ Gaithersburg, MD 20899
}

\section{Laboratory Participation summary}

The following table summarizes accreditation actions that have occurred during calendar year 1985. Since some laboratories are accredited in more than one field of testing, the number of accredited laboratories listed by field (see Index $B$ ) is greater than the number of laboratories in the system (see Index $A$ ).

\section{Field of Testing*}

TIM CON CAR STO ACO CPL DOS SEA ECT TOTAL

\begin{tabular}{|c|c|c|c|c|c|c|c|c|c|c|}
\hline Accreditations & & 1 & & & 1 & 2 & 10 & 1 & 14 & +27 \\
\hline Terminations & 3 & 3 & 1 & 2 & 1 & & 1 & & & -11 \\
\hline Suspensions & 1 & & & & & & & & & -1 \\
\hline Renewals & 1 & 2 & & & & & & & & +3 \\
\hline $\begin{array}{l}\text { Total Accredited } \\
\text { Laboratories }\end{array}$ & 35 & 28 & 21 & 9 & 7 & 6 & 44 & 1 & 14 & 165 \\
\hline $\begin{array}{l}\text { Change in Total } \\
\text { Accredited Labs } \\
\text { from December } \\
1984\end{array}$ & -2 & 0 & 0 & -2 & -1 & +2 & +9 & 0 & +14 & +20 \\
\hline $\begin{array}{l}\text { *TIM - Thermal Ir } \\
\text { CON - Concrete } \\
\text { CAR - Carpet } \\
\text { STO - Solid fue } \\
\text { ACO - Acoustics } \\
\text { CPL - Commercia } \\
\text { DOS - Radiation } \\
\text { SEA - Building } \\
\text { ECT - Electroma }\end{array}$ & $\begin{array}{l}\text { sime } \\
\text { ls } \\
\text { tic }\end{array}$ & $y$ & er & & ints, & & & & & ucts \\
\hline
\end{tabular}


The following Indexes list NLAP-accredited laboratories by NVLAP Code Number, laboratory name, city and state (or country if not the U.S.).

INDEX A. LISTING BY LABORATDRY NAME

INDEX B. LISTING BY FIELD OF TESTING

INDEX C. LISTING BY STATE 
INDEX A. LISTING BY LABORATORY NAME

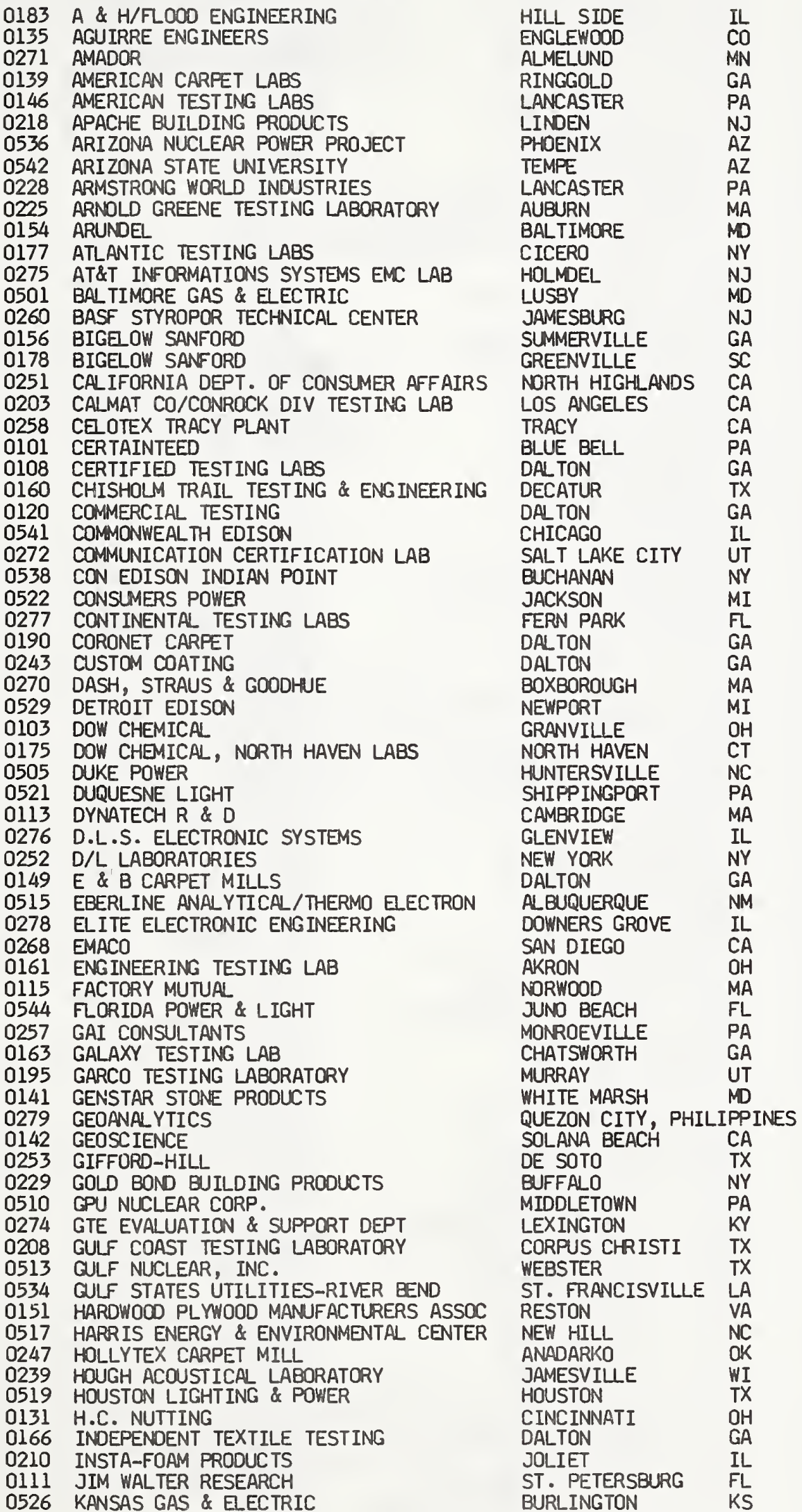


0248 KNAUF FIBER GLASS RESEARCH

0215 LINCOLN-DEVORE

0530 LOUISIANA POWER \& LIGHT CO

0259 MACMILL.AN BLOEDEL.

0503 MALLINCKRODT DIAGNDSTICS
0123 MANVILLE

0273 MET ELECTRICAL TESTING

0546 MISSISSIPPI POWER \& LIGHT

0104 NAHB RESEARCH FOUNDATION

0504 NAVAL MEDICAL COMMAND

0509 NAVAL RESEARCH LABORATORY

0543 NEW HAMPSHIRE YANKEE, SEABROOK STA

0508 NEW YORK POWER AUTHORITY-INDIAN POINT

0511 NEW YORK POWER AUTHORITY-LYCOMING

0269 NDRAND EMC TEST LAB

0540 NORTHEAST UTILITIES SERVICE

0244 NORTHWEST TESTING LABS

0525 OMAHA PUBLIC FOWER DISTRICT

0240 OMNI ENVIRONMENTAL SERVICES

0109 OWENS CORNING FIBERGLAS

0124 OWENS CORNING FIBERGLAS

0125 OWENS CORNING FIBERGLAS

0126 OWENS CORNING FIBERGLAS

0128 OMENS CORNING FIBERGLAS

0129 OWENS CORNING FIBERGLAS

0130 OWENS CORNING FIBERGLAS

0537 PACIFIC GAS \& ELECTRIC

0223 PFS CORPORATION

0237 PITTSBURGH TESTING LABORATORY

0201 PTL-INSPECTORATE

0531 PUBLIC SERVICE ELECTRIC \& GAS

$0280 R$ \& $B$ ENTERPRISES

0261 RADCO

0512 RADIATION DETECTION

0267 RETLIF TESTING LABORATORIES

0232 RITCHIE LABORATORIES

0227 RIVERBANK ACOUSTICAL LAB OF IIT

0514 ROCHESTER GAS \& ELECTRIC

0518 R. S. LANDAUER, JR/TECH OPSDIV

0245 R.F. GEISSER AND ASSOC

0206 R.W. SIDLEY

0221 SALEM CARPET LABORATORY

0193 SHAW INDUSTRIES

0264 SHELTON RESEARCH

0532 SIEMENS GAMMASONICS

0192 SMITH-EMERY

0547 SOUTH CAROLINA ELECTRIC \& GAS

0506 SOUTHERN CALIFORNIA EDISON

0121 SPARRELL ENGINEERING RESEARCH

0281 STANDARD T CHEMICAL/TECHNICAL CTR

0220

0233

0191

0122

0533

0516

0196

0528

0188

0116

0117

0255

0502

0105

0106

0107

0266

0241

0539

0216

0230

0520

0523

STRATTON LABORATORIES

STS CONSULTANTS

STS CONSULTANTS

TECHNICAL MICRONICS CONTROL

TELEDYNE ISOTOFES

TENNESSEE VALLEY AUTHORITY

TEXAS TESTING LABORATORY

TEXAS UTILITIES GENERATING

TWIN CITY TESTING AND ENGINEERING

UNDERWRITERS LABORATORIES

UNDERWRITERS LABORATORIES

UNDERWRITERS LABORATORIES

UNION ELECTRIC

UNITED STATES TESTING

UNITED STATES TESTING

UNITED STATES TESTING

UNITED STATES TESTING

UNITED STATES TESTING WESTERN STATES US ARMY IONIZING RADIATION DOS CTR USG

VIRGINIA CONCRETE LABORATORY

VIRGINIA ELECTRIC \& POWER, MINERAL VIRGINIA ELECTRIC \& POWER, SURRY

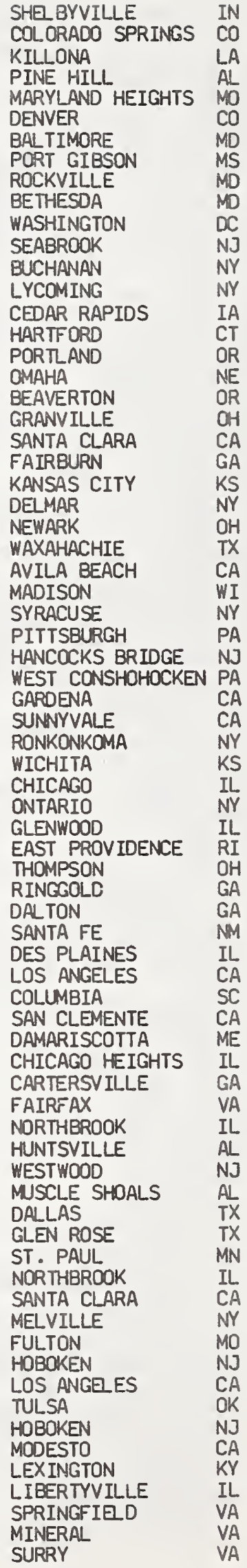


WALT KEELER

WICHITA

WARNOCK HERSEY

MIDOLETON

KS

0545

WASHINGTON PUBLIC POWER SUPPLY SYSTEM

RICHLAND

0256 WESTERN ELECTRO-ACOUSTIC LAB

0265 WEYERHAEUSER TECHNOLOGY CENTER

0263 WHITTAKER ANALYTICAL SERVICES

0226 WISS, JANNEY, ELSTNER AND ASSOCIATES

0197 WORLD CARPETS

0176 W. R. GRACE

0250 W. R. GRACE

0524 YANKEE ATOMIC ELCTRIC

SANTA MONICA CA

TACOMA WA

COLTON

NORTHBROOK

DALTON

CAMBRIDGE

CAMBRIDGE

FRAMINGHAM

CA

GA

MA

$M A$

MA

INDEX B. LISTING BY FIEID OF TESTING

acoustics

0111 JIM WALTER RESEARCH

0123 MANVILLE

0227 RIVERBANK ACOUSTICAL LAB OF IIT

0228 ARMSTRONG WORLD INDUSTRIES

O229 GOLD BOND BUILDING PRODUCTS

0239 HOUGH ACOUSTICAL LABORATORY

0256 WESTERN ELECTRO-ACOUSTIC LAB

carpet

0105 UNITED STATES TESTING

0106 UNITED STATES TESTING

0108 CERTIFIED TESTING LABS

0115 FACTORY MUTUAL

0120 COMMERCIAL TESTING

0139 AMERICAN CARPET LABS

0149 E \& B CARPET MILLS

0151 HARDWOOD PLWOCD MANUFACTURERS ASSOC

0156 BIGE OW SANFORD

0160 CHISHOLM TRAIL TESTING \& ENGINEERING

0163 GALAXY TESTING LAB

0166 INDEPENDENT TEXTILE TESTING

0178 BIGE OW SANFORD

0190 CORONET CARPET

0193 SHAW INDUSTRIES

0197 WORLD CARPETS

O220 STRATTON LABORATORIES

0221 SALEM CARPET LABORATORY

0243 CUSTOM COATING

0247 HOLLYTEX CARPET MILL

concrete

0131 H.C. NUTTING

0133 WALT KEELER

0135 AGUIRRE ENGINEERS

0141 GENSTAR STONE PRODUCTS

0146 AMERICAN TESTING LABS

0154 ARUNDEL

0161 ENGINEERING TESTING LAB

0176 W. R. GRACE

0177 ATLANTIC TESTING LABS

0183 A \& H/FLOOD ENGINEERING

0188 TWIN CITY TESTING AND ENGINEERING

0191 STS CONSULTANTS

0192 SMITH-EMERY

0195 GARCO TESTING LABORATORY

0196 TEXAS TESTING LABORATORY

0201 PTL-INSPECTORATE

O203 CALMAT CO/CONROCK DIV TESTING LAB

0206 R.W. SIDLEY

O208 GULF COAST TESTING LABORATORY

0215 LINCOLN-DEVORE

O230 VIRGINIA CONCRETE LABORATORY

0232 RITCHIE LABORATORIES

ST. PETERSBURG

DENVER

CHICAGO

LANCASTER

BUFFALO

JAMESVILLE

SANTA MONICA

FL

IL

PA

NY

WI

CA

HOBOKEN NJ

LOS ANGELES CA

DALTON GA

NORWOOD MA

DALTON GA

RINGGOLD GA

DALTON GA

RESTON VA

SUMMERVILLE GA

DECATUR TX

CHATSWORTH GA

DALTON GA

GREENVILLE SC

DALTON GA

DALTON GA

DALTON GA

CARTERSVILLE GA

RINGGOLD GA

DALTON GA

ANADARKO OK

CINCINNATI OH

WICHITA KS

ENGLEWOOD CO

WHITE MARSH MD

LANCASTER PA

BALTIMORE MD

AKRON $\mathrm{OH}$

CAMBRIDGE MA

CICERO NY

HILL SIDE IL

ST. PAUL MN

NORTHBROOK IL

LOS ANGELS CA

MURRAY UT

DALLAS TX

PITTSBURGH PA

LOS ANGELS CA

THOMPSON $\mathrm{OH}$

CORPUS CHRISTI TX

COLORADO SPRINGS CO

SPRINGFIED VA

WICHITA KS 
0233 STS CONSULTANTS

0237 PITTSBURGH TESTING LABORATORY

0241 UNITED STATES TESTING WESTERN STATES

0253 GIFFORD-HILL

0257 GAI CONSULTANTS

0279 GEOANALYTICS

FAIRFAX

SYRACUSE

VA

MODESTO

DE SOTO

MONROEVILLE

CA

QUEZON CITY,

$P A$

paint

0252

D/L LABORATORIES

0263 WHITTAKER ANALYTICAL SERVICES

0266 UNITED STATES TESTING

0281 STANDARD T CHEMICAL/TECHNICAL CTR

NEW YORK

COLTON

HOBOKEN

CHICAGO HEIGHTS

NY

$C A$

paper

0259

MACMILLAN BLOEDEL

0265 WEYERHAEUSER TECHNOLOGY CENTER

PINE HILL TACOMA

AL dosimetry

0501

BALTIMORE GAS \& ELECTRIC

UNION ELECTRIC

0502

MALLINCKRODT DIAGNOSTICS

0504 NAVAL MEDICAL COMMAND

0505 DUKE POWER

0506 SOUTHERN CALIFORNIA EDISON

0508 NEW YORK POWER AUTHORITY-INDIAN POINT

0509 NAVAL RESEARCH LABORATORY

0510 GPU NUCLEAR CORP.

0511 NEW YORK POWER AUTHORITY-LYCOMING

0512 RADIATION DETECTION

0513 GULF NUCLEAR, INC.

0514 ROCHESTER GAS \& ELECTRIC

0515 EBERLINE ANALYTICAL/THERMO ELECTRON

0516 TENNESSEE VALLEY AUTHORITY

0517 HARRIS ENERGY \& ENVIRONMENTAL CENTER

0518 R. S. LANDAUER, JR/TECH OPSDIV

0519 HOUSTON LIGHTING \& POWER

0520

0521

0522

0523

0524

0525

0526

0528

0529

0530

0531

0532

0533

0534

0536

0537

0538

0539

0540

0541
0543

0544

0545

0546

0542

VIRGINIA ELECTRIC \& POWER, MINERAL

DUQUESNE LIGHT

CONSUMERS POWER

VIRGINIA ELECTRIC \& POWER, SURRY

YANKEE ATOMIC ELECTRIC

OMAHA PUBLIC POWER DISTRICT

KANSAS GAS \& ELECTRIC

TEXAS UTILITIES GENERATING

DETROIT EDISON

LOUISIANA POWER \& LIGHT CO

PUBLIC SERVICE ELECTRIC \& GAS

SIEMENS GAMMASONICS

TELEDYNE ISOTOPES

GULF STATES UTILITIES-RIVER BEND

ARIZONA NUCLEAR POWER PROJECT

PACIFIC GAS \& ELECTRIC

CON EDISON INDIAN POINT

US ARMY IONIZING RADIATION DOS CTR

NORTHEAST UTILITIES SERVICE

COMMONWEALTH EDISON

NEW HAMPSHIRE YANKEE, SEABROOK STA

FLORIDA POWER \& LIGHT

WASHINGTON PUBLIC POWER SUPPLY SYSTEM

MISSISSIPPI POWER \& LIGHT

ARIZONA STATE UNIVERSITY

0547 SOUTH CAROLINA ELECTRIC \& GAS

electromagnetics

RETLIF TESTING LABORATORIES

EMACO

NORAND EMC TEST LAB

0271 AMADOR

$\begin{array}{lc}\text { LUSBY } & \text { MD } \\ \text { FULTON } & \text { MO } \\ \text { MARYLAND HEIGHTS } & \text { MO } \\ \text { BETHESDA } & \text { MD } \\ \text { HUNTERSVILLE } & \text { NC } \\ \text { SAN CLEMENTE } & \text { CA } \\ \text { BUCHANAN } & \text { NY } \\ \text { WASHINGTON } & \text { DC } \\ \text { MIDDLETOWN } & \text { PA } \\ \text { LYCOMING } & \text { NY } \\ \text { SUNNYVALE } & \text { CA } \\ \text { WEBSTER } & \text { TX } \\ \text { ONTARIO } & \text { NY } \\ \text { ALBUQUERQUE } & \text { NM } \\ \text { MUSCLE SHOALS } & \text { AL } \\ \text { NEW HILL } & \text { NC } \\ \text { GLENWOOD } & \text { IL } \\ \text { HOUSTON } & \text { TX } \\ \text { MINERAL } & \text { VA } \\ \text { SHIPPINGPORT } & \text { PA } \\ \text { JACKSON } & \text { MI } \\ \text { SURRY } & \text { VA } \\ \text { FRAMINGHAM } & \text { MA } \\ \text { OMAHA } & \text { NE } \\ \text { BURLINGTON } & \text { KS } \\ \text { GLEN ROSE } & \text { TX } \\ \text { NEWPORT } & \text { MI } \\ \text { KILLONA } & \text { LA } \\ \text { HANCOCKS BRIDGE } & \text { NJ } \\ \text { DES PLAINES } & \text { IL } \\ \text { WESTWOOD } & \text { NJ } \\ \text { ST. FRANCISVILLE } & \text { LA } \\ \text { PHOENIX } & \text { AZ } \\ \text { AVILA BEACH } & \text { CA } \\ \text { BUCHANAN } & \text { NY } \\ \text { LEXINGTON } & \text { KY } \\ \text { HARTTORD } & \text { CT } \\ \text { CHICAGO } & \text { IL } \\ \text { SEABROOK } & \text { NJ } \\ \text { JUNO BEACH } & \text { FL } \\ \text { RICHLAND } & \text { WA } \\ \text { PORT GIBSON } & \text { MS } \\ \text { TEMPE } & \\ \text { COLUMBIA } & \\ & \end{array}$

RONKONKOMA

SAN DIEGO

CEDAR RAPIDS

BOXBOROUGH

NY

ALMELUND

MD

MD

NC

NY

$D C$

PA

CA

NY

NM

AL

IL

VA

MI

VA

MA

KS

$M I$

A

IL

NJ

$A Z$

NY

KY

CT

NJ

WA

AZ

DASH, STRAUS \& GOODHUE

CA

IA

MA

MN 
0272 COMMUNICATION CERTIFICATION LAB

0255 UNDERWRITERS LABORATORIES

0273 MET ELECTRICAL TESTING

0275 AT\&T INFORMATIONS SYSTEMS EMC LAB

0274 GTE EVALUATION \& SUPPORT DEPT

0276 D.L.S. ELECTRONIC SYSTEMS

0277 CONTINENTAL TESTING LABS

0278 ELITE ELECTRONIC ENGINEERING

$0280 R$ \& $B$ ENTERPRISES

seals and sealants

0252

D/L LABORATORIES

thermal insulation

0116 UNDERWRI TERS LABORATORIES

0117 UNDERWRITERS LABORATORIES

0223 PFS CORPORATION

0225 ARNOLD GREENE TESTING LABORATORY

0240 OMNI ENVIRONMENTAL SERVICES

0244 NORTHWEST TESTING LABS

0245 R.F. GEISSER AND ASSOC

0249 WARNOCK HERSEY INT'L

0264 SHEL TON RESEARCH

0101 CERTAINTEED

0103 DOW CHEMICAL

0104 NAHB RESEARCH FOUNDATION

0105 UNI TED STATES TESTING

0106 UNITED STATES TESTING

0107 UNITED STATES TESTING

0109 OWENS CORNING FIBERGLAS

0111 JIM WALTER RESEARCH

0113 DYNATECH $R$ \& D

0115 FACTORY MUTUAL

0116 UNDERWRITERS LABORATORIES

0117 UNDERWRITERS LABORATORIES

0120 COMERCIAL TESTING

0121 SPARRELL ENGINEERING RESEARCH

0122 TECHNICAL MICRONICS CONTROL

0123 MANVIULE

0124 OWENS CORNING FIBERGLAS

0125 OWENS CORNING FIBERGLAS

0126 OWENS CORNING FIBERGLAS

O128 OWENS CORNING FIBERGLAS

0129 OWENS CORNING FIBERGLAS

0130 OWENS CORNING FIBERGLAS

0142 GEOSCIENCE

0175 DOW CHEMICAL, NORTH HAVEN LABS

0188 TWIN CITY TESTING AND ENGINEERING

0210 INSTA-FOAM PRODUCTS

0216 USG

0218 APACHE BUILDING PRODUCTS

0226 WISS, JANNEY, ELSTNER AND ASSOCIATES

0248 KNAUF FIBER GLASS RESEARCH

0250 W. R. GRACE

0251 CALIFORNIA DEPT. OF CONSUMER AFFAIRS

0258 CELOTEX TRACY PLANT

0260 BASF STYROPOR TECHNICAL CENTER

0261 RADCO

\section{INDEX C. LISTING BY STATE}

0122 TECHNICAL MICRONICS CONTROL

0259

0516

0536

0542

0106

0117

0124

0142

0192
MACMILLAN BLOEDEL

TENNESSEE VALLEY AUTHORITY

ARIZONA NUCLEAR POWER PROJECT

ARIZONA STATE UNIVERSITY

UNITED STATES TESTING

UNDERWRI TERS LABORATORIES

OWENS CORNING FIBERGLAS

GEOSCIENCE

SMITH-EMERY
SALT LAKE CITY

MELVILLE

LEXINGTON KY

GLENVIEW

FERN PARK FL

DOWNERS GROVE IL

WEST CONSHOHOCKEN PA

NEW YORK

NY

NORTHBROOK IL

SANTA CLARA CA

MADISON WI

AUBURN MA

BEAVERTON OR

PORTLAND OR

EAST PROVIDENCE RI

MIODLETON WI

SANTA FE NM

BLUE BELL PA

GRANVILLE $\quad \mathrm{OH}$

ROCKVILLE MD

HOBOKEN NJ

LOS ANGELES CA

TULSA OK

GRANVILLE $\quad \mathrm{OH}$

ST. PETERSBURG FL

CAMBRIDGE MA

NORWOOD MA

NORTHBROOK IL

SANTA CLARA CA

DALTON GA

DAMARISCOTTA ME

HUNTSVILLE AL

DENVER CO

SANTA CLARA CA

FAIRBURN GA

KANSAS CITY KS

DEMAR NY

NEWARK $\quad \mathrm{OH}$

WAXAHACHIE TX

SOLANA BEACH CA

NORTH HAVEN CT

ST. PAUL MN

JOLIET IL

LIBERTYVILLE IL

LINDEN NJ

NORTHBROOK IL

SHELBYVILLE IN

CAMBRIDGE MA

NORTH HIGHLANDS CA

TRACY CA

JAMESBURG NJ

GARDENA CA

HUNTSVILLE AL

PINE HILL AI

MUSCLE SHDALS AL

PHOENIX AZ

TEMPE $\quad A Z$

LOS ANGELES CA

SANTA CLARA CA

SANTA CLARA CA

SOLANA BEACH CA

LOS ANGELES CA 
0203

0241

0251

0256

0258

0506

0512

0537

0261

0263

0268

0123

0135

0215

0175

0540

0509

0111

0544

0277

0108

0120

0125

0139

0149

0156

0163

0166

0190

0193

0197

0220

0221

0243

0269

0116

0183

0191

0210

0216

0226

0227

0518

0532

0541

0276

0278

0281

0248

0126

0133

0232

0526

0539

0274

0530

0534

0113

0115

0176

0250

0225

0524

0270

0104

0141

0154

0501

0504

0273

0121

0522

0529
CALMAT CO/CONROCK DIV TESTING LAB UNITED STATES TESTING WESTERN STATES CALIFORNIA DEPT. OF CONSUMER AFFAIRS WESTERN ELECTRO-ACOUSTIC LAB

CELOTEX TRACY PLANT

SOUTHERN CALIFORNIA EDISON

RADIATION DETECTION

PACIFIC GAS \& ELECTRIC

RADCO

WHITTAKER ANALYTICAL SERVICES

EMACO

MANVILLE

AGUIRRE ENGINEERS

LINCOLN-DEVORE

DOW CHEMICAL, NORTH HAVEN LABS

NORTHEAST UTILITIES SERVICE

NAVAL RESEARCH LABORATORY

JIM WALTER RESEARCH

FLORIDA POWER \& LIGHT

CONTINENTAL TESTING LABS

CERTIFIED TESTING LABS

COMMERCIAL TESTING

OWENS CORNING FIBERGLAS

AMERICAN CARPET LABS

$E$ \& B CARPET MILLS

RIGELOW SANFORD

GALAXY TESTING LAB

INDEPENDENT TEXTILE TESTING

CORONET CARPET

SHAW INDUSTRIES

WORLD CARPETS

STRATTON LABORATORIES

SALEM CARPET LABORATORY

CUSTOM COATING

NDRAND EMC TEST LAB

UNDERWRITERS LABORATORIES

A \& HFLOOO ENGINEERING

STS CONSULTANTS

INSTA-FOAM PRODUCTS

USG

WISS, JANNEY, ELSTNER AND ASSOCIATES

RIVERBANK ACOUSTICAL LAB OF IIT

R. S. LANDAUER, JR/ TECH OPSDIV

SIEMENS GAMMASONICS

COMMONWEALTH EDISON

D.L.S. ELECTRONIC SYSTEMS

EITE ELECTRONIC ENGINEERING

STANDARD T CHEMICAL/TECHNICAL CTR

KNAUF FIEER GLASS RESEARCH

OWENS CORNING FIBERGLAS

WALT KEELER

RITCHIE LABORATORIES

KANSAS GAS \& ELECTRIC

US ARMY IONIZING RADIATION DOS CTR

GTE EVALUATION \& SUPPORT DEPT

LOUISIANA POWER \& LIGHT CO

GULF STATES UTILITIES-RIVER EEND

DYNATECH $R$ \& D

FACTORY MUTUAL

W. R. GRACE

W. R. GRACE

ARNOLD GREENE TESTING LABORATORY

YANKEE ATOMIC ELECTRIC

DASH, STRAUS \& GOODHUE

NAHB RESEARCH FOUNDATION

GENSTAR STONE PRODUCTS

ARUNDE

BALTIMORE GAS \& ELECTRIC

NAVAL MEDICAL COMMAND

MET ELECTRICAL TESTING

SPARREL ENGINEERING RESEARCH

CONSUMERS POWER

DETROIT EDISON
LOS ANGELES

MODESTO

NDRTH HIGHLANDS

SANTA MONICA

TRACY

SAN CLEMENTE

SUNNYVALE

AVILA BEACH

GAROENA

COLTON

SAN DIEGO

DENVER

ENGLEWOOD

COLORADO SPRINGS

NORTH HAVEN

HARTFORD

WASHINGTON

ST. PETERSBURG

JUND BEACH

FERN PARK

DALTON

DALTON

FAIRBURN

RINGGOLD

DALTON

SUMMERVILLE

CHATSWORTH

DALTON

DALTON

DALTON

DAL TON

CARTERSVILLE

RINGGOLD

DALTON

CEDAR RAPIDS

NOR THBROOK

HILL SIDE

NORTHBRDOK

JOLIET

LIBERTYVILLE

NDRTHBROOK

CHICAGO

GLENWOOD

DES PLAINES

CHICACO

GLENVIEW

DOWNERS GROVE

CHICACO HEIGHTS

SHE BYVILLE

KANSAS CITY

WICHITA

WICHITA

BURLINGTON

LEXINGTON

LEXINGTON

KILLONA

ST . FRANCISVILLE

CAMBRIDGE

NORWOOD

CAMBR IDGE

CAMBRIDGE

AUBURN

FRAMINGHAM

BOXBOROUGH

ROCKVILE

WHITE MARSH

BAL TIMORE

LUSBY

BETHESDA

BALT IMORE

DAMARISCOTTA

JACKSON

NEWPORT
CA

$C A$

$C A$

CA

$C A$

$C A$

$C A$

$C A$

$C A$

$C A$

$\mathrm{CO}$

CO

CO

C

FL

FL

FL

GA

$G A$

GA

GA

GA

GA

GA

GA

GA

GA

GA

GA

GA

IA

IL

IL

IL

IL

IL

IL

IL

I

IL

IL

IN

KS

KS

KS

KS

KY

KY

LA

LA

MA

$M A$

MA

$M A$

MA

$M A$

$M A$

MD

$M D$

MD

MD

MD

MD

ME

MI

MI 
TWIN CITY TESTING AND ENGINEERING

ST. PAUL

0271 AMADOR

0502 UNION ELECTRIC

0503 MALLINCKRODT OIAGNOSTICS

0546 MISSISSIPPI POWER \& LIGHT

0505 DUKE POWER

0517 HARRIS ENERGY \& ENVIRONMENTAL CENTER

0525

0105

0266

OMAHA PUBLIC POWER DISTRICT

UNITED STATES TESTING

UNITED STATES TESTING

0218 APACHE BUILDING PRODUCTS

0531 PUBLIC SERVICE ELECTRIC \& GAS

0533 TELEDYNE ISOTOPES

0543 NEW HAMPSHIRE YANKEE, SEABROOK STA

O260 BASF STYROPOR TECHNICAL CENTER

0275 AT\&T INFORMATIONS SYSTEMS EMC LAB

0515 EBERLINE ANALYTICAL/THERMO EECTRON

0264 SHELTON RESEARCH

0128 OWENS CORNING FIBERGLAS

0177 ATLANTIC TESTING LABS

0229 GOLD BOND BUILOING PROOUCTS

0237 PITTSBURGH TESTING LABORATORY

0252 O/L LABORATORIES

0508 NEW YORK POWER AUTHORITY-INDIAN POINT

0511 NEW YORK POWER AUTHORITY-LYCOMING

0514 ROCHESTER GAS \& ELECTRIC

0538 CON EDISON INDIAN POINT

0252 O/L LABORATORIES

0267 RETLIF TESTING LABORATORIES

0255 UNDERWRITERS LABORATORIES

0103 DOW CHEMICAL

0109 OWENS CORNING FIBERGLAS

0129 OWENS CORNING FIBERGLAS

0131 H.C. NUTTING

0161 ENGINEERING TESTING LAB

0206 R.W. SIDLEY

0107 UNITED STATES TESTING

0247 HOLLYTEX CARPET MILL

0240 OMNI ENVIRONMENTAL SERVICES

0244 NORTHWEST TESTING LABS

0101 CERTAINTEEO

0146 AMERICAN TESTING LABS

0201 PTL-INSPECTORATE

0228 ARMSTRONG WORLD INDUSTRIES

0257 GAI CONSULTANTS

0510 GPU NUCLEAR CORP.

0521 DUQUESNE LIGHT

$0280 R$ \& $B$ ENTERPRISES

0245 R.F. GEISSER AND ASSOC

0178 BIGELOW SANFORD

0547 SOUTH CAROLINA EIECTRIC \& GAS

O130 OWENS CORNING FIBERGLAS

0160 CHISHOLM TRAIL TESTING \& ENGINEERING

0196 TEXAS TESTING LABORATORY

0208 GULF COAST TESTING LABORATORY

0253 GIFFORD-HILI

0513 GULF NUCLEAR, INC.

0519 HOUSTON LIGHTING \& POWER

0528 TEXAS UTILITIES GENERATING

O195 GARCO TESTING LABORATORY

0272 COMMUNICATION CERTIFICATION LAB

0151 HARDWOOD PL YWOOO MANUFACTURERS ASSOC

0230 VIRGINIA CONCRETE LABORATORY

0233 STS CONSULTANTS

0520 VIRGINIA EECTRIC \& POWER, MINERAL

0523 VIRGINIA ELECTRIC \& POWER, SURRY

0545 WASHINGTON PUBLIC POWER SUPPLY SYSTEM

0265 WEYERHAEUSER TECHNOLOGY CENTER

0223 PFS CORPORATION

0239 HOUGH ACOUSTICAL LABORATORY

0249 WARNOCK HERSEY INT'L

0279 GEOANALYTICS

ALMELUND MN

FULTON

MARYLAND HEIGHTS M

PORT GIBSON MS

HUNTERSVILLE NC

NEW HILL NC

OMAHA NOBOKEN NE

HOBOKEN NJ

LINDEN NJ

HANCOCKS BRIDGE NJ

WESTWOOD NJ

SEABROOK NJ

JAMESBURG NJ

HOL MOEL NJ

ALBUQUERQUE NM

SANTA FE NM

DEMAR NY

CICERO NY

BUFFALO NY

SYRACUSE NY

NEW YORK NY

BUCHANAN NY

LYCOMING NY

ONTARIO NY

BUCHANAN NY

NEW YORK NY

RONKONKOMA NY

MELVILLE NY

GRANVILLE $\quad O H$

GRANVILLE OH

NEWARK $\mathrm{OH}$

CINCINNATI $\quad \mathrm{OH}$

AKPON $\quad \mathrm{OH}$

THOMPSON OH

TULSA OK

ANADARKO OK

BEAVERTON OR

PORTLAND OR

BLUE BELL $\quad P A$

LANCASTER PA

PITTSBURGH PA

LANCASTER PA

MONROEVILLE $\quad P A$

MIDDLETOWN PA

SHIPPINGPORT PA

WEST CONSHOHOCKEN PA

EAST PROVIOENCE RI

GREENVILLE SC

COLLMBIA SC

WAXAHACHIE TX

DECATUR TX

DALLAS TX

CORPUS CHRISTI TX

OE SOTO TX

WEBSTER TX

HOUSTON TX

GLEN POSE TX

MURRAY UT

SALT LAKE CITY UT

RESTON VA

SPRINGFIED VA

FAIRFAX VA

MINERAL VA

SURRY VA

RICHLAND WA

TACOMA WA

MADISON WI

JAMESVILLE WI

MIDDLETON WI

QUEZON CITY, PHILIPPINES 
Index D. Sumary of Accredited Laboratories by Test Method and Field of Testing.

\section{ACOUSTICS}

NVLAP Test Method Code Number

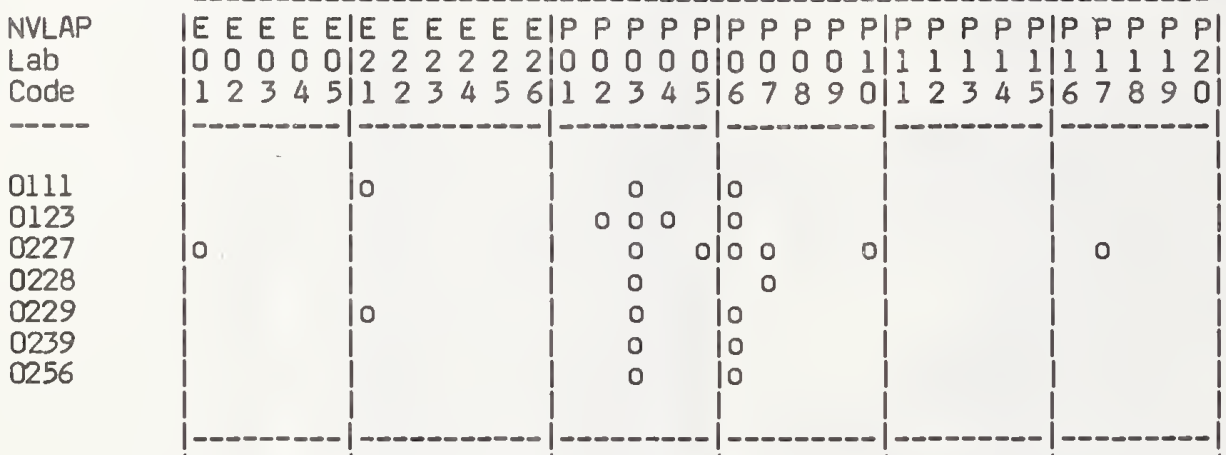

|E E E EIE E E E EIP P P P PIP P P P PIP P P P PIP P P P

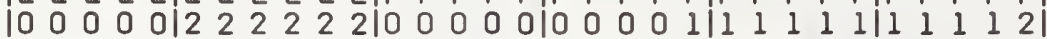

$112345|123456| 12345|67890| 12345 \mid 678901$

\section{CARPET}

NVLAP Test Method Code Number

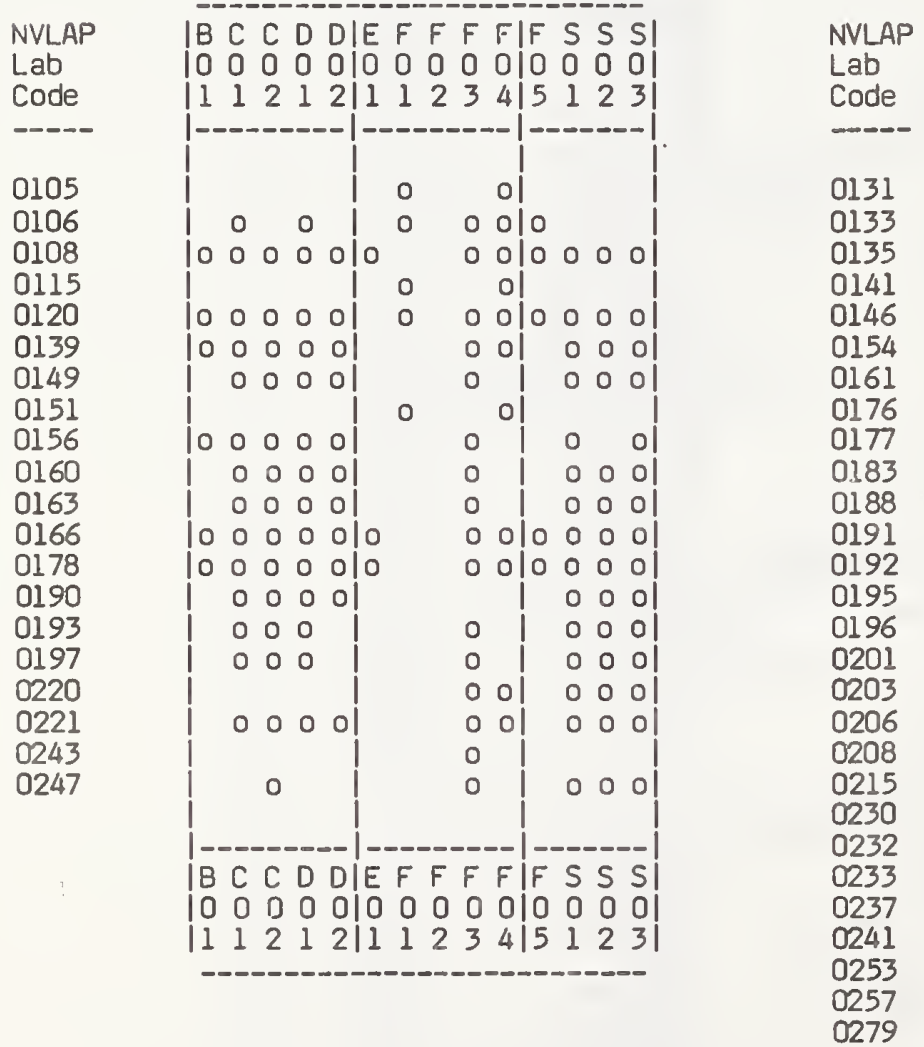

\section{CONCRETE}

NVLAP Test Method Code Number

G A A A AIA A A A AIA A A A AIA A A $\left|\begin{array}{llllllllllllllllllllll}0 & 0 & 0 & 0 & 0 & 0 & 0 & 0 & 0 & 0 & 1 & 1 & 1 & 1 & 1 & 1 & 1 & 1\end{array}\right|$

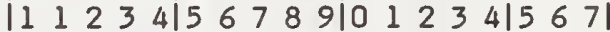

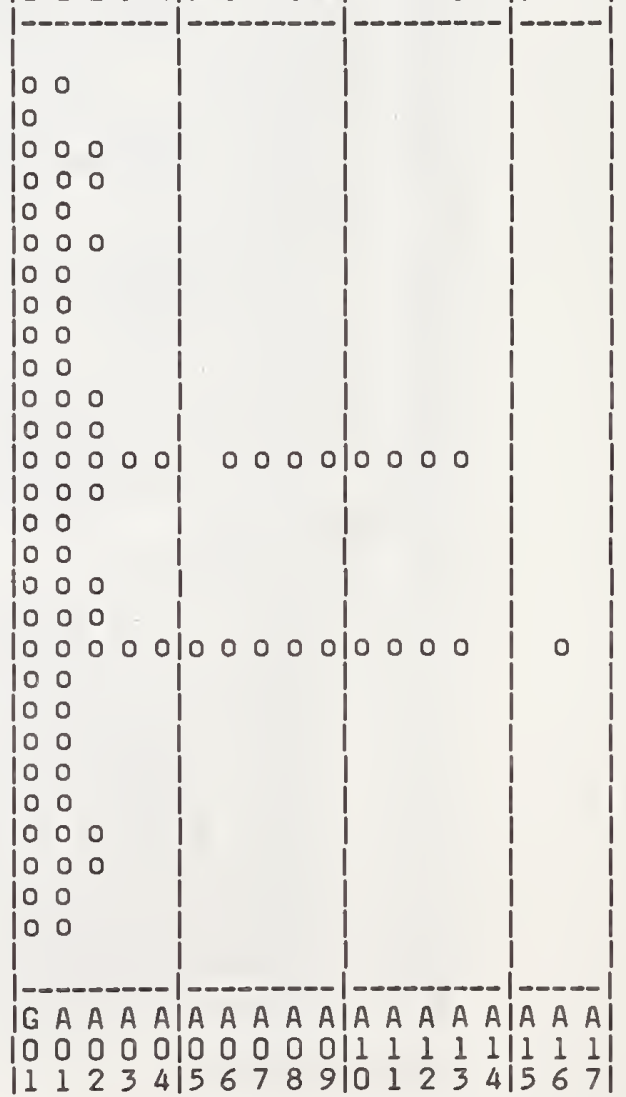


DOSIMETRY

\begin{tabular}{|c|c|c|c|c|c|c|c|c|}
\hline \multirow{2}{*}{$\begin{array}{l}\text { NVLAP } \\
\text { Lab } \\
\text { Code }\end{array}$} & & ANST $\mathrm{N} 13,1$ & 3.1 & \multicolumn{5}{|c|}{ Categories (see note) } \\
\hline & I & II & III & IV & V & VI & VII & VIII \\
\hline 0501 & & 0 & & 0 & 0 & & 0 & 0 \\
\hline 0502 & & 0 & & & & 0 & 0 & 0 \\
\hline 0503 & & & & & & & 0 & \\
\hline 0504 & & 0 & 0 & 0 & 0 & 0 & 0 & 0 \\
\hline 0505 & & $\mathbf{0}$ & & o & o & & 0 & \\
\hline 0506 & 0 & 0 & 0 & 0 & 0 & 0 & 0 & \\
\hline 0508 & 0 & 0 & 0 & 0 & 0 & 0 & 0 & \\
\hline 0509 & & 0 & 0 & $\mathbf{0}$ & 0 & 0 & 0 & 0 \\
\hline 0510 & 0 & 0 & 0 & 0 & 0 & 0 & 0 & 0 \\
\hline 0511 & & o & & 0 & & 0 & 0 & \\
\hline 0512 & 0 & 0 & 0 & 0 & 0 & 0 & 0 & 0 \\
\hline 0513 & 0 & 0 & 0 & 0 & 0 & 0 & 0 & 0 \\
\hline 0514 & 0 & 0 & 0 & 0 & 0 & 0 & 0 & 0 \\
\hline 0515 & 0 & 0 & 0 & 0 & 0 & 0 & 0 & 0 \\
\hline 0516 & 0 & 0 & 0 & 0 & 0 & 0 & 0 & 0 \\
\hline 0517 & 0 & 0 & 0 & 0 & 0 & 0 & 0 & 0 \\
\hline 0518 & 0 & 0 & 0 & 0 & 0 & 0 & 0 & 0 \\
\hline 0519 & & 0 & & 0 & & & 0 & \\
\hline 0520 & & 0 & & 0 & 0 & & 0 & \\
\hline 0521 & 0 & 0 & 0 & 0 & 0 & 0 & 0 & \\
\hline 0522 & & 0 & & 0 & 0 & & 0 & 0 \\
\hline 0523 & & 0 & & 0 & 0 & & 0 & \\
\hline 0524 & 0 & 0 & 0 & 0 & 0 & 0 & 0 & 0 \\
\hline 0525 & & 0 & & 0 & 0 & & 0 & 0 \\
\hline 0526 & & 0 & 0 & 0 & 0 & 0 & 0 & 0 \\
\hline 0528 & 0 & 0 & 0 & 0 & 0 & 0 & 0 & 0 \\
\hline 0529 & & & 0 & 0 & 0 & 0 & 0 & 0 \\
\hline 0530 & 0 & 0 & 0 & 0 & 0 & 0 & 0 & 0 \\
\hline 0531 & 0 & 0 & 0 & 0 & 0 & 0 & 0 & 0 \\
\hline 0532 & 0 & 0 & 0 & 0 & 0 & 0 & 0 & 0 \\
\hline 0533 & 0 & 0 & 0 & 0 & 0 & 0 & 0 & 0 \\
\hline 0534 & 0 & 0 & 0 & 0 & 0 & 0 & 0 & 0 \\
\hline 0536 & 0 & 0 & 0 & 0 & 0 & 0 & 0 & 0 \\
\hline 0537 & & 0 & 0 & 0 & 0 & 0 & 0 & 0 \\
\hline 0538 & & 0 & 0 & 0 & 0 & 0 & 0 & 0 \\
\hline 0539 & 0 & 0 & 0 & 0 & 0 & 0 & 0 & 0 \\
\hline 0540 & & 0 & & 0 & 0 & 0 & 0 & 0 \\
\hline 0541 & 0 & 0 & 0 & 0 & 0 & 0 & 0 & \\
\hline 0542 & & & & 0 & & & & \\
\hline 0543 & 0 & 0 & 0 & 0 & 0 & 0 & 0 & 0 \\
\hline 0544 & 0 & 0 & 0 & 0 & 0 & 0 & 0 & 0 \\
\hline 0545 & & 0 & & 0 & 0 & & 0 & 0 \\
\hline 0546 & 0 & 0 & 0 & 0 & 0 & 0 & 0 & 0 \\
\hline 0547 & 0 & 0 & 0 & 0 & 0 & 0 & 0 & 0 \\
\hline
\end{tabular}

\section{ELCTROMAGNTICS}

NVLAP Test Method Code Number

\begin{tabular}{|c|c|}
\hline $\begin{array}{l}\text { NVLAP } \\
\text { Lab } \\
\text { Code }\end{array}$ & $\left|\begin{array}{lllll}C & R & T & T & T \\
0 & 0 & 0 & 0 & 0 \\
1 & 1 & 1 & 2 & 3\end{array}\right|$ \\
\hline $\begin{array}{l}0255 \\
0267 \\
0268 \\
0269 \\
0270 \\
0271 \\
0272 \\
0273 \\
0274 \\
0275 \\
0276 \\
0277 \\
0278 \\
0280\end{array}$ & $\left|\begin{array}{lllll}0 & 0 & & & \\
0 & 0 & 0 & 0 & 0\end{array}\right|$ \\
\hline & $\left|\begin{array}{lllll}C & R & T & T & T \\
0 & 0 & 0 & 0 & 0 \\
1 & 1 & 1 & 2 & 3\end{array}\right|$ \\
\hline
\end{tabular}

NOTE: Processors may be accredited for more than one dosimeter type. See the Scope of Accreditation for each processor in the last section of the Directory for details. 


\section{INSULATION}

NVLAP Test Method Code Number

NVLAP IC C CDDIDDDDDIDDDDDIDDDDDIDDDDDIDDDDDI

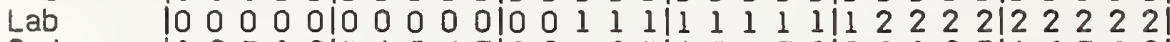

Code $\quad 112312134567 \mid 89123145678190123346789$

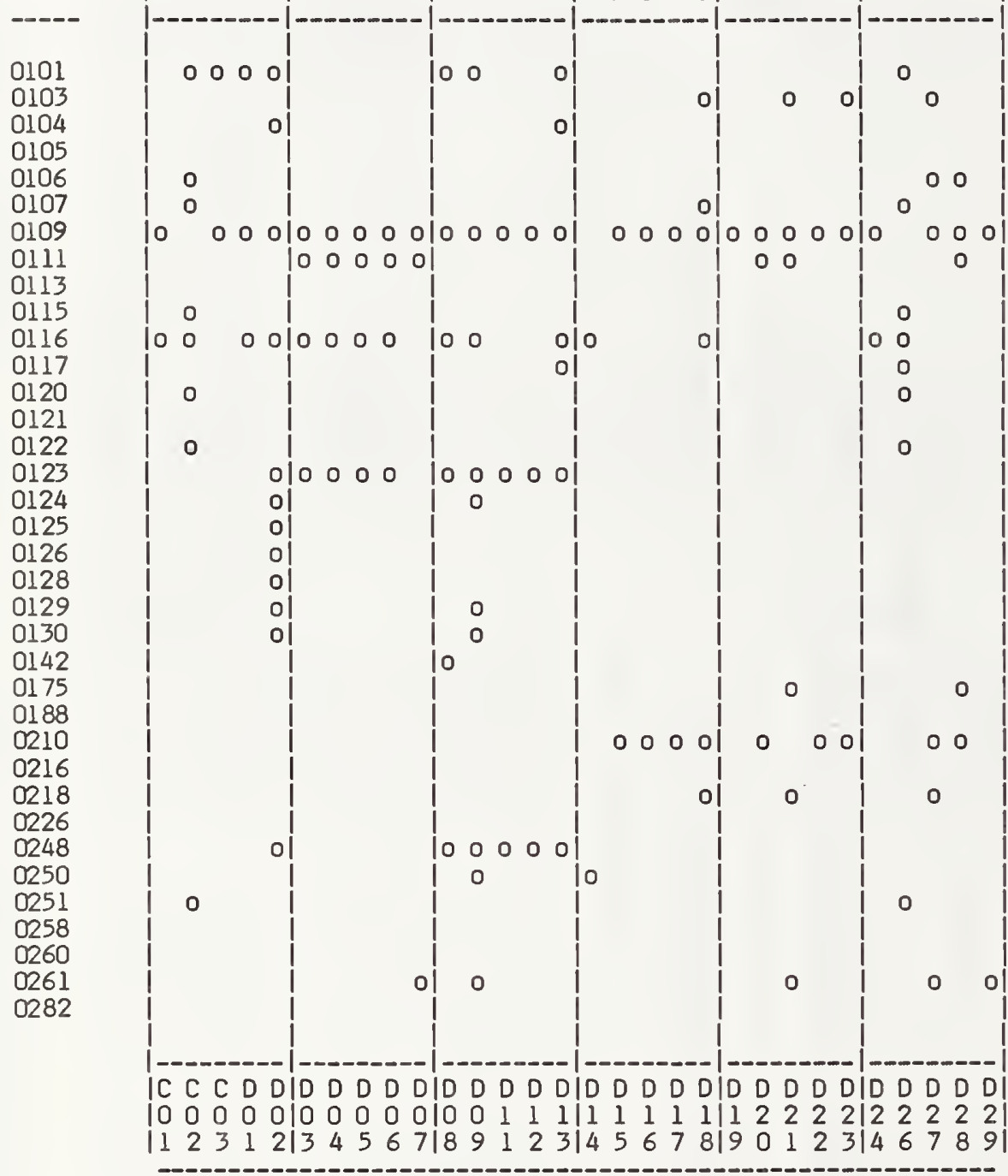

continued next page 
BSUATION (continued)

NVLAP Test Method Code Number

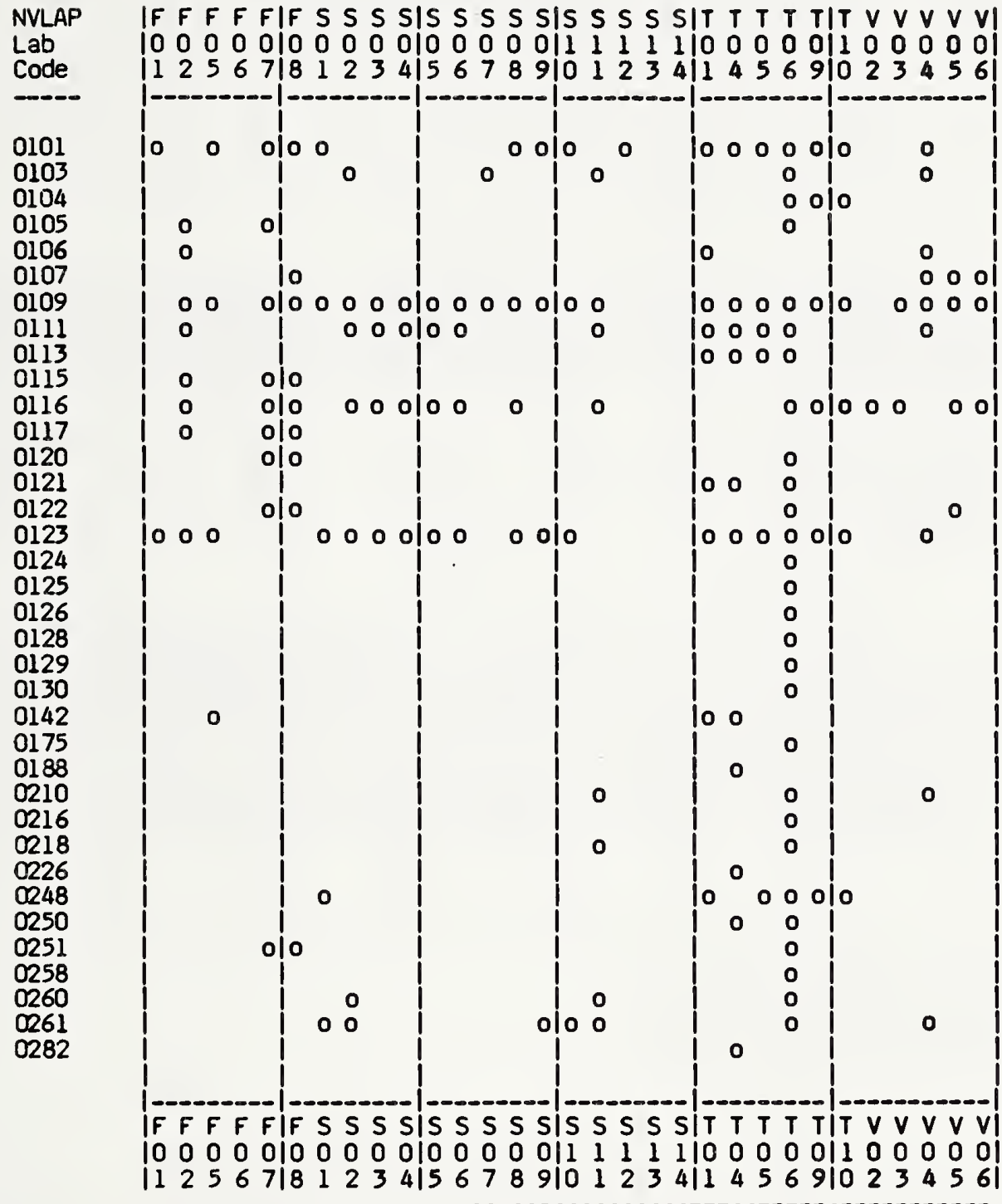




\section{PAINTS AND RELATED COATINES AND MATERIALS}

NVLAP Test Method Code Number

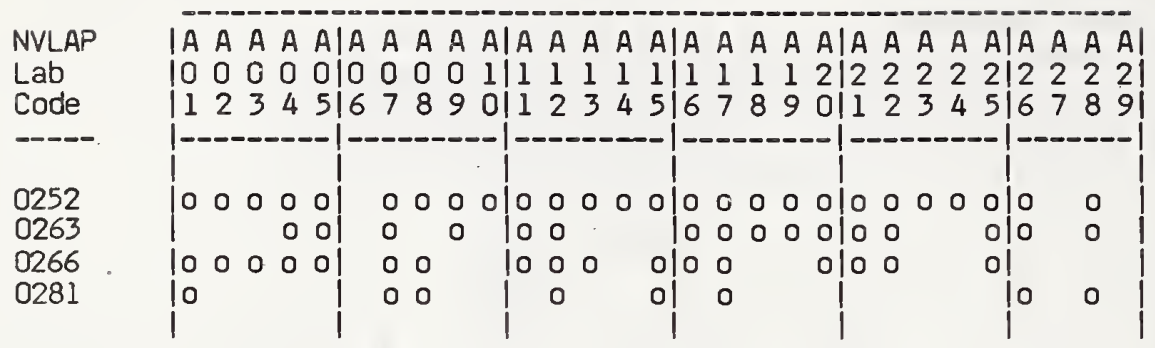

NVLAP

Lab

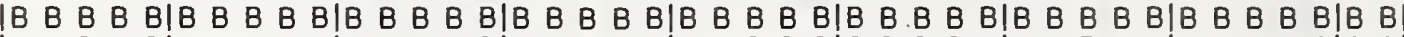

Code

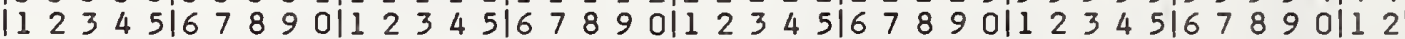

0252

0263

0266

0281

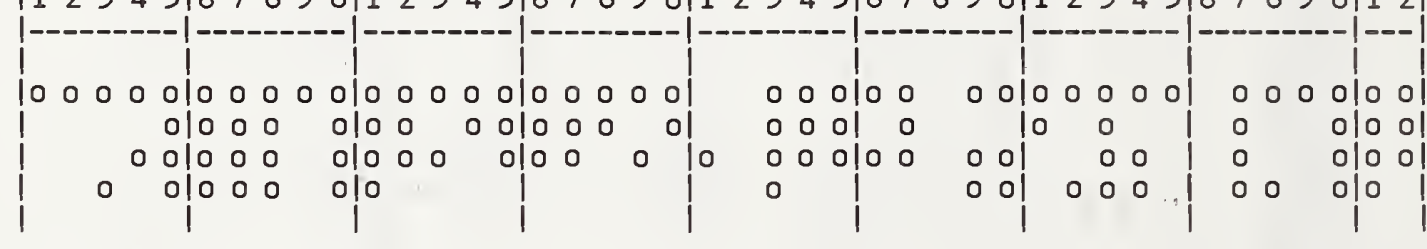

NVLAP

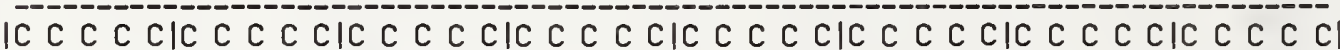

Lab

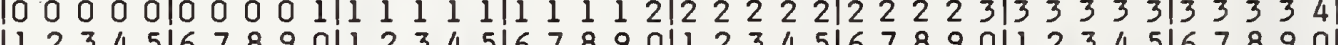

Code

0252

0263

0266

0281

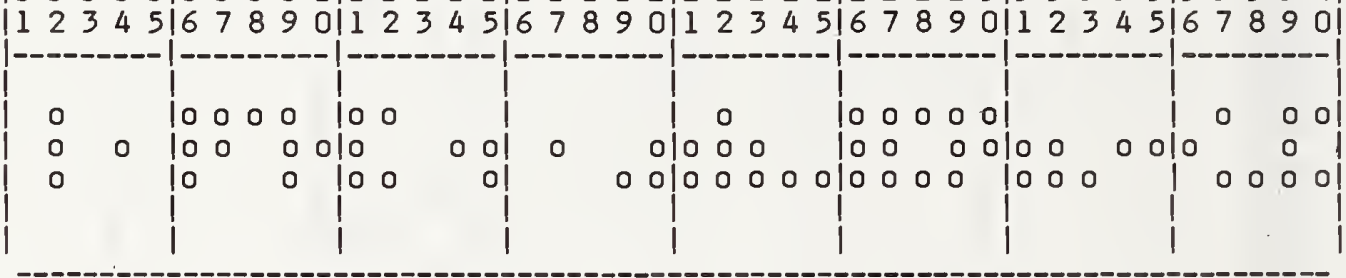

NVLAP

Lab

Code

ID D D D DIODODDIODDODOI

----

0252

0263

0266

0281

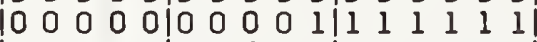

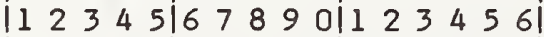

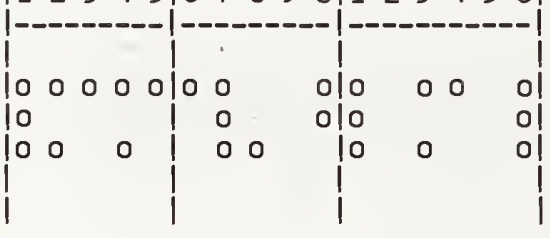




\section{PAPER AND RELATED PRODUCTS}

NVLAP Test Method Code Number
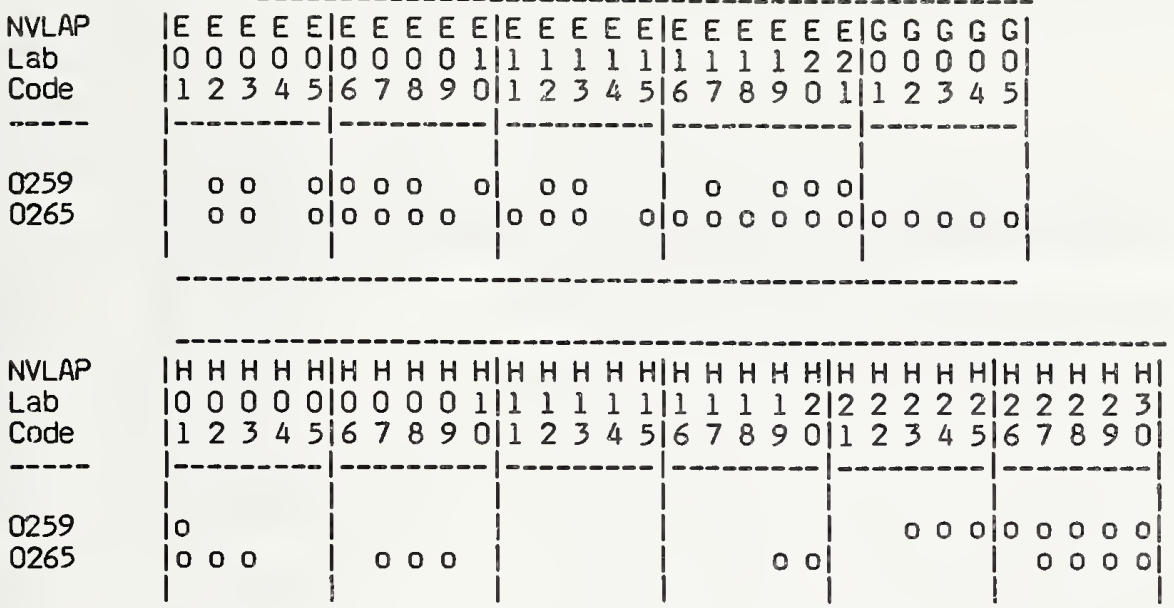

\section{SEPLS ADD SEPLANTS}

NVLAP Test Method Code Number

NVLAP

Lab

Code

----

0252

1000001000001000001000001000001000001

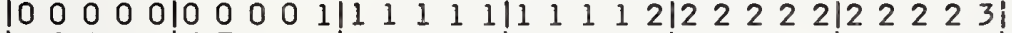

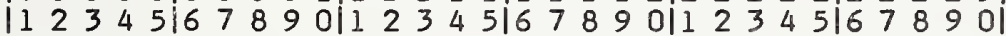

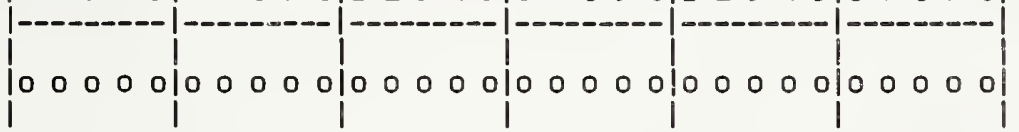

STOVE

NVLAP Test Method Code Number

NVLAP

Lab

Code

IE E E EIE E E E EIEE E F FIF F F FIF F F FIF F F F M M M M M M

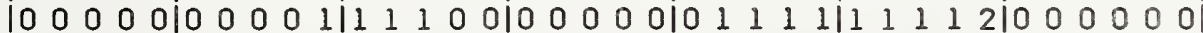
$1123451678900112312 \mid 456781901244678901123456$

----

0116

0117

0223

0225

0240

0244

0245

0249

0264

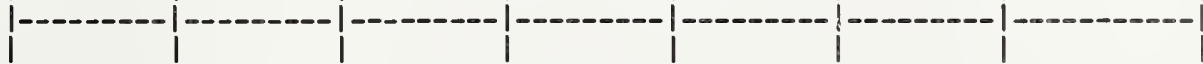

100000 10 0 o 0 o

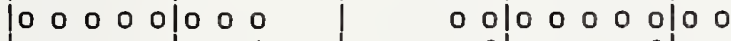

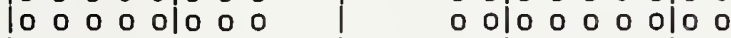

$10 \begin{array}{lll}0 & 0 & 0 \\ 0 & 0 & 0\end{array}$

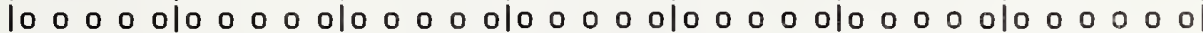
10000001000
0 ollo 000 o
1000

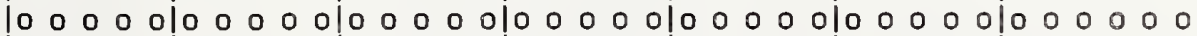

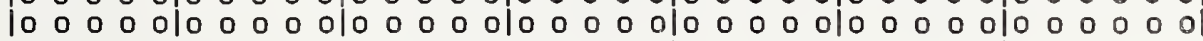

1000001000

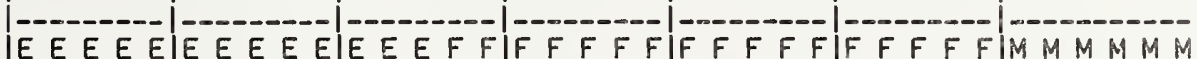
$1000000 \mid 0000111111001000001011111111111210000000$

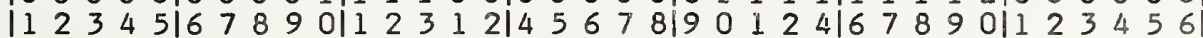


INDEX E. ACCREDITED LABORATORIES ANO TEST METHDOS FOR WHICH THEY ARE ACCREDITED 
CERTAINTEED CORPORATION

INSULATION GROUP, R \& D LABORATORY

1400 Union Neeting Road, Blue Be11, PA 19422

Dr. H. Francis 011x phone: $215-341-6713$

Accreditation Renewal [ate: January 1, 1988

\begin{tabular}{|c|c|c|}
\hline NVLAP Code & Designation & Short ritle \\
\hline $\begin{array}{l}01 / \mathrm{CO} 2 \\
01 / \mathrm{CO} 3\end{array}$ & $\begin{array}{l}16 \text { CFR-Part } 1209.5 \\
\text { California Energy Commiss } \\
\text { Corrosivenass - Mineral }\end{array}$ & $\begin{array}{l}\text { Corrosiveness; Cellulosic fiber (loose-fill) } \\
\text { sion tests for insulating materials: } \\
\text { fiber blankets and loose-fill }\end{array}$ \\
\hline $01 / 001$ & ASTM C136 & Sieve or screen analysis \\
\hline $01 / 002$ & ASTM Cl67 & Thickness and density; Blanket and batt \\
\hline $01 / 008$ & ASTM C302 & Density: Preformed pipe insulation \\
\hline $01 / 009$ & ASTM C303 & Density: Preformed block insulation \\
\hline $01 / 013$ & ASTM C 519 & Density; Loose-fill. (fibrous) \\
\hline $01 / 026$ & 16 CFR-Part 1209.4 & Settled density; cellulosic fiber (loose-piII) \\
\hline $01 / F 01$ & TAPPI T461 & Flame Resistance; Paper and paperboard \\
\hline $01 / F 05$ & ASTM E 136 & Behavior of Materials in a Vertical Tube Furnace \\
\hline $01 / F 07$ & 16 CFR-Part 1209.6 & $\begin{array}{l}\text { Critical Iadiant flux; Radiant Panel } \\
\text { (cellulosic fiber, loose-fili) }\end{array}$ \\
\hline $01 / F 08$ & 16 CFR-Part & Smoldering combustion; Cellulosic fiber (loose-fill) \\
\hline $01 / \mathrm{SO1}$ & ASTM Cl65 & Compressive properties: Themal insulation (proc. A) \\
\hline $01 / 508$ & ASTM C446 & Breaking load/modulus of rupture; Preformed pipe insulation \\
\hline $01 / 509$ & ASTM D781 & Puncture test; Paperboard and fiberboard \\
\hline $01 / 510$ & ASTM D828 & Tensile breaking strength: Paper and paperbcard \\
\hline $01 / 512$ & California Energy Commiss & $\begin{array}{l}\text { ssion tests for insulating materials: } \\
\text { Bond strength - Spray applied cellulose }\end{array}$ \\
\hline $01 / \mathrm{TOl}$ & ASTM $C 177$ & $\begin{array}{l}\text { Thermal transmission properties: } \\
\text { Low-temperature guarded hot. plate }\end{array}$ \\
\hline $01 / \mathrm{TO4}$ & ASTM C236 & Thermal conductance; Guarded hot box \\
\hline $01 / 705$ & ASTM $C 335$ & Thermal conductivity; Pipe insulation \\
\hline $01 /$ TOS & ASTM C518 & Thermal transmission properties; heat flow meter \\
\hline $01 /$ T09 & ASTM C 653 & Thermal resistance (Rec. Practice): Blanket (mineral fiber) \\
\hline & ASTM C687 & Thermal resistance (Rec. Practice); Loose-fill (fibrous) \\
\hline & ASTM E96 & Hater vapor transmission; Thin sheets (proc. A) \\
\hline
\end{tabular}

NVLAP LAB CODE OLO3

DOW CHEMICAL USA, FOAN PRODUCTS RESEARCH

PRODUCT EVALUATION GROUP

P.D. Box 515, Gragville, of 43023

Pike J. Ennis Phone: 614-587-4215

Accreditation Renewal Date: January 1, 1988

\begin{tabular}{|c|c|}
\hline NMLAP Code & Designation \\
\hline $\begin{array}{l}01 / 018 \\
01 / 021\end{array}$ & $\begin{array}{l}\text { ASTM D1622 } \\
\text { ASTM D2126 }\end{array}$ \\
\hline $\begin{array}{l}01 / 023 \\
01 / 027\end{array}$ & $\begin{array}{l}\text { ASTM D2842 } \\
\text { ASTM D2126 }\end{array}$ \\
\hline $\begin{array}{l}01 / \mathrm{SO2} \\
01 / \mathrm{SO7} \\
01 / \mathrm{S} 11\end{array}$ & $\begin{array}{l}\text { ASTM C203 } \\
\text { ASTM C } 273 \\
\text { ASTM D } 1621\end{array}$ \\
\hline $\begin{array}{l}\text { 01/T06 } \\
01 / \text { V04 }\end{array}$ & $\begin{array}{l}\text { ASTM C518 } \\
\text { ASTM E96 }\end{array}$ \\
\hline
\end{tabular}

Short Yitle

Apparent density: Rigid cellular plastics

Response to thermal and humid aging

(proc. E); Rigid cellular plastics

water absorption; Rigid cellular plastics

Response to thermal and humid aging

(proc. c); Rigid cellular plastics

Breaking load/flexural strength: Preformed block insulation

Shear test: Sandwich construction

Compressive properties: Rigid cellular plastics

(proc. A-Crosshead)

Thermal transmission properties; Heat flow meter

Water vapor transmission; Thin sheets (proc. A) 
NAHB RESEARCH FOUNDATION, INC.

627 Southlawn Lane, Rockville, MD 20850

Hugh Angleton Phone: 301-762-4200

Accreditation Renewal Date: January 1, 1988

\begin{tabular}{cll} 
NYLAP Code & & Designation \\
\cline { 1 - 2 } $01 / 002$ & & ASTM C167 \\
$01 / 013$ & & ASTM C519 \\
$01 / T 06$ & & ASTM C518 \\
$01 / T 09$ & & ASTM C 653 \\
$01 / T 10$ & & ASTM C 687
\end{tabular}

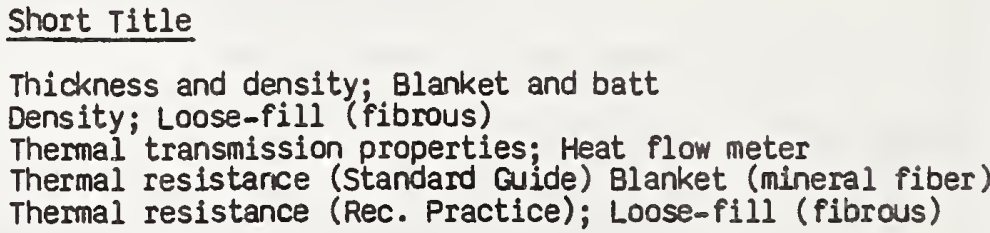

Short Title

Thickness and density; Blanket and batt Density; Loose-fill (fibrous)

Thermal transmission properties; Heat flow meter

Thermal resistance (Standard Guide) Blanket (mineral fiber)

Thermal resistance (Rec. Practice); Loose-fill (fibrous)

NVLAP LAB CODE 0105

UNITED STATES TESTING COMPANY, INC.

ENGINEERING SERVICES DIVISION

291 Fairfield Avenue, Fairfield, NJ 07006

Rudolph Giglio Phone: 201-575-5252

\begin{tabular}{|c|c|}
\hline NVAP Code & Designation \\
\hline $\begin{array}{l}01 / F 02 \\
01 / F 07\end{array}$ & $\begin{array}{l}\text { ASTM E84 } \\
16 \text { CFR-Part } 1209.6\end{array}$ \\
\hline $\begin{array}{l}\text { 01/T06 } \\
\text { 03/F01 } \\
\text { 03/F04 }\end{array}$ & $\begin{array}{l}\text { ASTM C518 } \\
\text { ASTM E84 } \\
\text { ASTM E648 }\end{array}$ \\
\hline
\end{tabular}

NVAP LAB CODE 0106

\author{
Short Title \\ Surface buming characteristics; Building materials \\ Critical radiant flux; \\ Radiant Panel (cellulosic fiber, loose-fill) \\ Thermal transmission properties; Heat flow meter \\ Surface Flamability (Carpets) \\ Radiant Panel (carpet)
}

UNITED STATES TESTING COMPANY, INC.

CALIFORNIA DIVISION

5555 Telegraph Road, Los Angeles, CA 90040

Bernd Givon Phone: 213-723-7181

Accreditation Renewal Date: January 1, 1988

\begin{tabular}{|c|c|}
\hline VAP Code & Designation \\
\hline $\begin{array}{l}01 / \mathrm{CO} 2 \\
\text { (Fo } \\
01 / 027\end{array}$ & $\begin{array}{l}16 \text { CFR-Part } 1209.5 \\
\text { ly HH-I-515 Part } 4.8 .5) \\
\text { ASTM D } 2126\end{array}$ \\
\hline $01 / D 28$ & ASTM D2126 \\
\hline $\begin{array}{l}01 / F 02 \\
01 / T_{01}\end{array}$ & $\begin{array}{l}\text { ASTM E } 84 \\
\text { ASTM C177 }\end{array}$ \\
\hline $\begin{array}{l}01 / \mathrm{VO4} \\
03 / \mathrm{CO1} \\
03 / 001\end{array}$ & $\begin{array}{l}\text { ASTM E96 } \\
\text { AATCC } 16 E \\
\text { ASTM D } 418\end{array}$ \\
\hline $\begin{array}{l}03 / F 01 \\
03 / F 03 \\
03 / F 04 \\
03 / F 05\end{array}$ & $\begin{array}{l}\text { ASTM E84 } \\
16 \text { CFR Part } 1630 \\
\text { ASTM E648 } \\
\text { ASTM E662 }\end{array}$ \\
\hline
\end{tabular}

\title{
Short Title
}

Corrosiveness; Cellulosic fiber (loose-fill)

Response to thermal and humid aging (proc. C); Rigid cellular plastics Response to thermal and humid aging (proc. G); Rigid cellular plastics Surface burning characteristics; Building materials Thermal transmission properties: Low-temperature guarded hot plate Water vapor transmission: Thin sheets (proc. A)

Color fastness to Light (Xenon Arc)

Pile Yam Floor Covering Construction

Pile Weight - Uncoated (Section 8)

Pile Weight - coated (Section 9)

Pile Thickness - (Sections 10 \& 11)

Tuft Height - (Section 13)

Surface Flammability

Methenamine Pill Test (FF 1-70) Sec.1630.4

Radiant Panel (Carpet)

optical Density of Smoke Generated by Solid Materials 
UNITED STATES TESTING COMPANY, INC. TULSA DIVISION

1341 North 108th East Avenue, Tulsa, OK 74116 Carl Yoder Phone: 918-437-8333

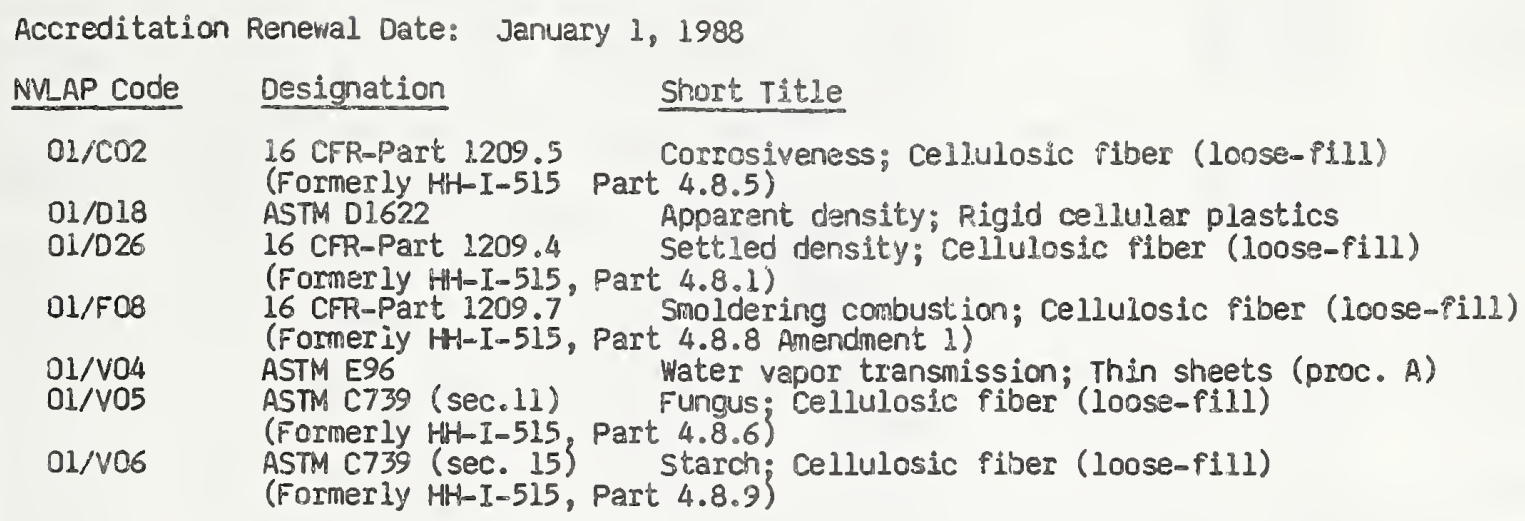

NMLAP LAB CODE 0108

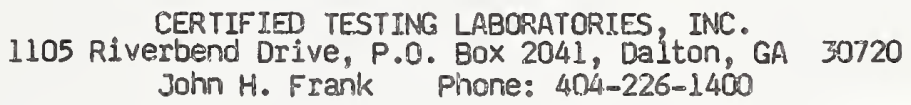

Accreditation Renewal Date: January 1, 1988

\begin{tabular}{ll} 
NVLAP Code & Designation \\
\hline $03 / B 01$ & UM44d (Table 5) \\
$03 / C 01$ & AATCC 16E \\
$03 / C 02$ & AATCC 8 \\
$03 / D 01$ & ASTM D 418
\end{tabular}

$\begin{array}{ll}03 / 002 & \text { DOD-C-95A } \\ 03 / \text { EO1 } & \text { AATCC 134/CRI 102 } \\ 03 / F 03 & \text { 16 CFR Part 1630 } \\ & \text { (FF 1-70) SEC.1630.4 } \\ 03 / F 04 & \text { ASTM E648 } \\ 03 / F 05 & \text { ASTM E662 } \\ 03 / 501 & \text { ASTM D1335 } \\ 03 / 502 & \text { ASTM D2646, seC.7. } \\ 03 / 503 & \text { ASTM D3936 }\end{array}$

\section{Short Title}

Attached Cushion Tests Colorfastness to Light (Xenon Arc) colorfastness to Crocking Pile Varn floor covering construction Pile Weight - Uncoated (Section 8) Pile Veight - coated (Sectjon 9) pile Thickness - (Sections 10 \& 11) Tuft Height - (Section 13) Shrinkage Electrostatic Propensity of Carpets Methenamine Pill Test

\author{
Radiant Panel (Carpet) \\ optical Density of Smoke Generated by Solid Materials \\ Tuft Bind of Floor Coverings \\ Testing Backing Fabrics, Breaking Load \\ Delamination strength of Secondary \\ Backing of Pile Floor Coverings
}

NKLAP LAB CODE 0109

\author{
OWENS-CORNING FIBERGLAS CORPORATION \\ TECHNICAL CENTER LABORATORY \\ P.0. Box 415, Route 16, Granville, OH 43023 \\ William M. Edmunds Phone: 614-587-7024
}

Accreditation Renewal Date: January 1, 1988

$\begin{array}{lll}\text { NVAP Code } & \text { Designation } & \text { Short Title } \\ \text { 01/CO1 } & \text { ASTM C739 (sec.9) } & \text { Corrosiveness; Cellulosic Tiber (loose-fill) } \\ 01 / C 03 & \text { California Energy Commission tests for insulating materials: } \\ 01 / 001 & \text { ASTM C136 } & \text { Corrosiveness - Mineral fiber blankets and loose-fill } \\ 01 / 002 & \text { ASTM C167 } & \text { Sieve or screen analysis }\end{array}$


01/003

$01 / 004$

$01 / 005$

01/006

$01 / 007$

$01 / 008$

$01 / 009$

01/011

$01 / 012$

$01 / 013$

$01 / 015$

$01 / 016$

$01 / 017$

$01 / 018$

$01 / 019$

01/D20

$01 / 021$

$01 / 022$

$01 / 023$

$01 / 024$

$01 / 027$

$01 / 028$

$01 / 029$

01/F02

01/F05

$01 /$ FO 7

01/F08

$01 / 501$

$01 / 502$

$01 / 503$

$01 / 504$

$01 / 505$

$01 / 506$

$01 / 507$

$01 / 508$

$01 / 509$

$01 / 510$

01/S11

01/TO1

01/TO4

01/T05

$01 /$ T06

01/TO9

$01 / T 10$

01/V03

$01 / V_{04}$

01/V05

01/V06
ASTM C209 (sec. 6)

ASTM C209 (sec. 13)

ASTM C209 (sec.13)

by 01037 ( $\mathrm{sec}$. 100-106)

ASTM C209 (sec. 14)

by 01037 ( $\mathrm{sec}, 107-110$ )

ASTM C272

ASTM C 302

ASTM C 303

ASTM C356

ASTM C411

ASTM C 519

ASTM 0756

ASTM 0756

ASTM 0756

ASTM D1622

ASTM D2126

ASTM 02126

ASTM 02126

ASTM 02126

ASTM 02842

ASTM C739 (sec. 12)

ASTM D2126

ASTM D2126

California Energy Commiss

ASTM E84

ASTM E136

16 CFR-Part 1209.6

16 CFR-Part 1209.7

(Formerly HH-I-5i5, part 4.8.8)

ASTM C203

ASTM C209 (sec.9)

ASTM C209 (sec. 10)

ASTM C209 (sec. 11)

ASTM C209 (sec. 12)

ASTM C273

ASTM C446

ASTM 0781

ASTM D828

ASTM D1621

ASTM $\mathrm{C} 177$

ASTM C236

ASTM C 335

ASTM C518

ASTM $C 653$

ASTM $\mathrm{C} 687$

ASTM DT487

ASTM E96

ASTM C739 (sec. 11)

(Formerly H-I-515,

ASTM C739 (sec. 15) Starch;
(Formerly HH-I-515, part 4.8.9) art 4.8.6

4.8.9\}
Thickness; Board (cellulosic fiber)

Water absorption, 2 hour;

Water absorption, 24 hour;

Board (cellulosic fiber)

Linear expansion:

Board (cellulosic fiber)

Density; Preformed block insulation

Density; Preformed pipe insulation

Density; Preformed block insulation

Linear shrinkage; Soaking heat;

Preformed high temperature insulation

Hot-surface performance; High temperature insulation

Density; Loose-fill (fibrous)

Weight and shape changes; Accelerated service (proc. A); Plastics

Weight and shape changes; Accelerated service (proc. B); Plastics

Weight and shape changes; Accelerated service (proc. E); Plastics

Apparent density; Rigid cellular plastics

Response to thermal and humid aging

(proc. B); Rigid cellular plastics

Response to thermal and humid aging (proc. D); Rigid cellular plastics

Response to thermal and humid aging (proc. E); Rigid cellular plastics

Response to thermal and humid aging (proc. F); Rigid cellular plastics

Water absorption; Rigid cellular plastics

Moisture absorption; Cellulosic fiber (loose-fill)

Response to thermal and humid aging (proc. C); Rigid cellular plastics

Response to thermal and humid aging (proc. G); Rigid cellular plastics

on tests for insulating materials:

surface buming characteristics; Building materials

Behavior of Materials in a Vertical Tube Furnace

Critical radiant flux; (formerly H-I-515, Part 4.8..7) Radiant Panel (cellulosic fiber,

Smoldering combustion; Cellulosic fiber (loose-fill)

Compressive properties; Thermal insulation (proc. A)

Breaking load/flexural strength; Preformed block insulation

Transverse strength; Board (cellulosic fiber)

Deflection at specified load; Board (cellulosic fiber)

Tensile strength; Parallel to surface;

Board (cellulosic fiber)

Tensile strength; Perpendicular to surface

Shear test; Sandwich construction

Breaking load/modulus of rupture; Preformed pipe insulation

Puncture test; Paperboard and fiberboard

Tensile breaking strength; Paper and paperboard

Compressive properties; Rigid cellular plastics (proc. A-Crosshead)

Thermal transmission properties; Low-temperature guarded hot plate

Thermal conductance; Guarded hot box

Thermal conductivity; Pipe insulation

Thermal transmission properties; Heat flow meter

Thermal resistance (Rec. Practice); Blanket (mineral fiber)

Thermal resistance (Rec. Practice); Loose-fill (fibrous)

Mildew (fungus) resistance; Paper and paperboard

Water vapor transmission; Thin sheets (proc. A)

Fungus; Cellulosic fiber (loose-fill)

Starch; Cellulosic fiber (loose-fill) 
JIM WALTER RESEARCH CORFORATION

10301 9th Street North, St. Petersburg, FL 33702 John $E$. Sheridan Phone: $813-576-4171$

Accreditation Renewal Date: Jaruary 1, 1988

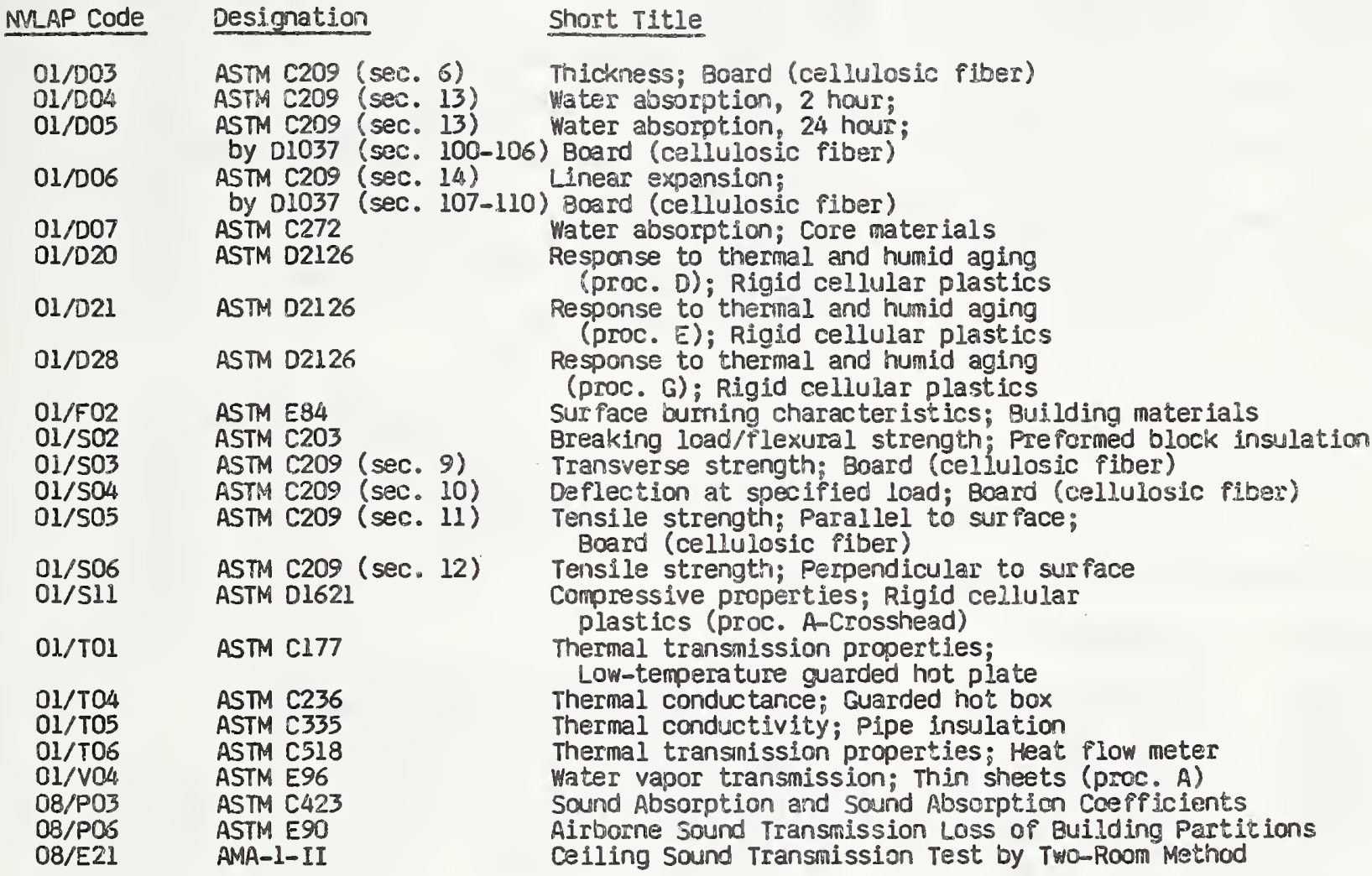

NVLAP LAB CODE 0113

DYNATECH R/D COMPANY

THERMOPHYSICS LABORATORY

99 Erie Street, Cambridge, MA 02139

Andre 0. Desjarlais Phone: 617-868-8050

Accreditation Renewal Date: January 1, 1988

\begin{tabular}{|c|c|}
\hline MLAP Code & Designati \\
\hline 01/T01 & ASTM C177 \\
\hline $\begin{array}{l}\text { 01/T04 } \\
01 / T 05 \\
\text { O1/T06 }\end{array}$ & $\begin{array}{l}\text { ASTM C236 } \\
\text { ASTM C335 } \\
\text { ASTM C518 }\end{array}$ \\
\hline
\end{tabular}

Short Title

Thermal transmission properties;

Low-temperature guarded hot plate

Thermal conductance; Guarded hot box

Thermal conductivity; Pipe insulation

Thermal transmission properties: Heat flow meter 
FACTORY MUTUAL RESEARCH CORPCRATION

1151 Boston-PTovidence Tumpike, Norwood, MA 02062

William F. Maroni Phone: $617-762-4300$

Accreditation Renewal Date: January 1, 1988

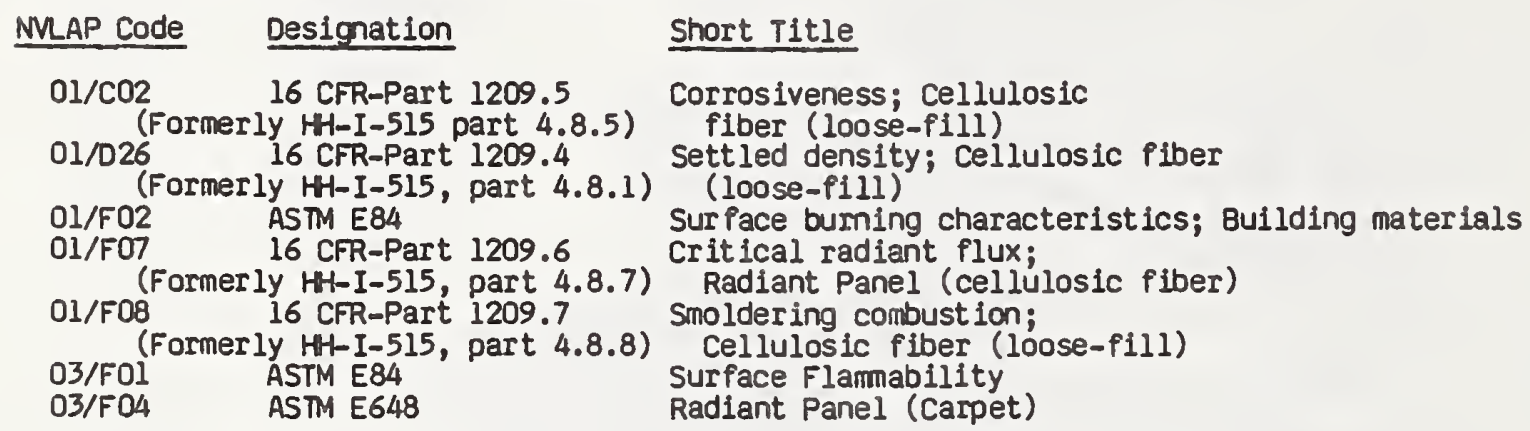

NVLAP LAB CODE 0116

UNDERWRITERS LABORATORIES INC.

333 Pfingsten Road, Northbrook, IL 60062

Steve Mazzoni Phone: 312-272-8800

Accreditation Renewal Date: January 1, 1988

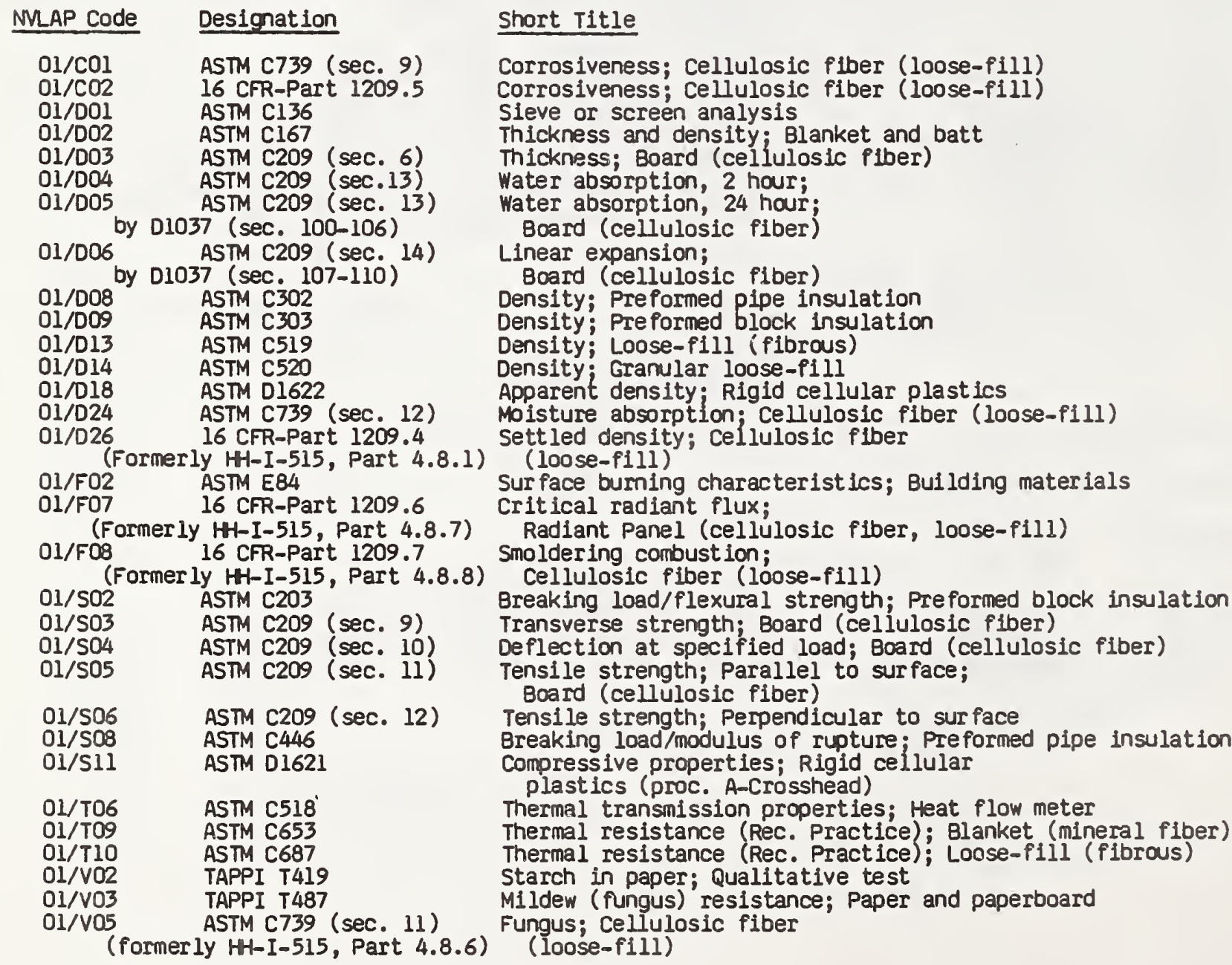


01/V06 ASTM C739 (sec. 15) Starch; Cellulosic fiber
(formerly H-I-515, Part 4.8.9) (loose-fill)

PAYSICAL/FIRE TEST GROUP (04/FOO)

\begin{tabular}{|c|c|}
\hline NMLAP Code & Short Title \\
\hline $\begin{array}{l}04 / F 01 \\
04 / F 02 \\
04 / F 04 \\
04 / F 05 \\
04 / F 06 \\
04 / F 07 \\
04 / F 08 \\
04 / F 09 \\
04 / F 10\end{array}$ & $\begin{array}{l}\text { Test Installation } \\
\text { Temperature Measurement } \\
\text { Radiant Fire Test } \\
\text { Coal Fire Test } \\
\text { Brand Fire Test } \\
\text { Flash Fire Test } \\
\text { Strength Tests } \\
\text { Stability Test } \\
\text { Glazing Test }\end{array}$ \\
\hline
\end{tabular}

$\begin{array}{cc}\begin{array}{c}\text { Section of UL } 737 \\ \text { 5th Edition }\end{array} & \text { Section of UL } 1482 \\ \text { 2nd Edition } \\ \text { (November 9, 1982) } & \text { (January 24, 1983) }\end{array}$

Section of CSA Standard B 366.2 +41984

(ULC S627-M1984)

(April, 1984)

$\begin{array}{lll}\text { 04/F11 } & \text { Test Installation } & 7.2 \\ \text { 04/F12 } & \text { Temperature Measurement } & 7.3 \\ \text { 04/F14 } & \text { Radiant Fire Test } & 7.5 \\ 04 / F 16 & \text { Brand Fire Test } & 7.6 \\ 04 / F 17 & \text { Flash Fire Test } & 7.7 \\ 04 / F 18 & \text { Strength Tests } & 7.12 \\ \text { 04/F19 } & \text { Stability Test } & 7.10 \\ \text { 04/F20 } & \text { Glazing Test } & 7.11\end{array}$

MOBILE HOME TEST GROUP (04/MOO)

\begin{tabular}{|c|c|c|c|}
\hline NVLAP Code & Short Title & $\begin{array}{c}\text { Section of UL } 737 \\
\text { sth Edition } \\
\text { (November 9, 1982) }\end{array}$ & $\begin{array}{c}\text { Section of UL } 1482 \\
\text { 2nd Edition } \\
\text { (January 24, 1983) }\end{array}$ \\
\hline $\begin{array}{l}\text { O4/MO1 } \\
04 / \mathrm{MO2} \\
04 / \mathrm{MO3}\end{array}$ & $\begin{array}{l}\text { Test Installation } \\
\text { Toxic Gas } \\
\text { Drop Test }\end{array}$ & $\begin{array}{l}17 \\
17 \\
17\end{array}$ & $\begin{array}{l}17 \\
17 \\
17\end{array}$ \\
\hline
\end{tabular}

Section of CSA Standard B 366.2 M1984

(ULC S627-Mi984)

(Apri1, 1984)

$\begin{array}{lll}\text { 04/MO4 } & \text { Test Installation } & 12 \\ \text { 04/MO5 } & \text { Toxic Gas } & 12 \\ \text { 04/MO6 } & \text { Drop Test } & 12\end{array}$

ELECTRICAL TEST GROUP (04/EOO)

\begin{tabular}{|c|c|c|c|}
\hline NVLAP Code & Short Title & $\begin{array}{c}\text { Section of Ul } 737 \\
\text { 5th Edition } \\
\text { (November 9, 1982) }\end{array}$ & $\begin{array}{c}\text { Section of UL } 1482 \\
\text { 2nd Edition } \\
\text { (January 24, 1983) }\end{array}$ \\
\hline $\begin{array}{l}\text { O4/EO1 } \\
\text { O4/E02 }\end{array}$ & $\begin{array}{l}\text { Test Voltages } \\
\text { Temperature Measurements, } \\
\text { Electrical Components }\end{array}$ & $\begin{array}{l}33 \\
34\end{array}$ & $\begin{array}{l}33 \\
34\end{array}$ \\
\hline $\begin{array}{l}\text { O4/EO3 } \\
\text { O4/E.04 }\end{array}$ & $\begin{array}{l}\text { Input Test } \\
\text { Temperature Test, } \\
\text { Electrical Components }\end{array}$ & $\begin{array}{l}35 \\
36\end{array}$ & $\begin{array}{l}35 \\
36\end{array}$ \\
\hline $\begin{array}{l}\text { O4/EO5 } \\
04 / E 06 \\
04 / E 07\end{array}$ & $\begin{array}{l}\text { Leakage Current } \\
\text { Dielectr ic Withstand } \\
\text { Locked Rotor (Stalled } \\
\text { Motor) Temperature }\end{array}$ & $\begin{array}{l}39 \\
37 \\
39\end{array}$ & $\begin{array}{l}38 \\
37 \\
39\end{array}$ \\
\hline 04/E08 & Power Cord Strain Relief & 40 & 40 \\
\hline
\end{tabular}




\begin{tabular}{|c|c|c|c|}
\hline & & $\begin{array}{c}\text { Section of CSA } \\
\text { C } 22.2 \text { No. } 3 \\
1979 \\
\end{array}$ & $\begin{array}{l}\text { Section of CSA } \\
\text { C 22. } 2 \text { No. } 113 \\
1982\end{array}$ \\
\hline O4/EO9 & $\begin{array}{l}\text { Temperature Measurements, } \\
\text { Electrical Components }\end{array}$ & 6.4 & 6.2 \\
\hline O4/E1O & $\begin{array}{l}\text { Temperature Test, } \\
\text { Electrical Components }\end{array}$ & 6.4 & 6.2 \\
\hline $\begin{array}{l}\text { O4/E11 } \\
\text { O4/E12 }\end{array}$ & $\begin{array}{l}\text { Leakage Current } \\
\text { Dielectric withstand }\end{array}$ & $\begin{array}{l}6.8 \\
6.5\end{array}$ & $\begin{array}{l}6.3 \\
6.3\end{array}$ \\
\hline O4/E13 & Power Cord Strain Relief 6 & & \\
\hline
\end{tabular}

NVLAP LAB COOE 0117

Accreditation Renewal Date: January 1, 1988

UNDERWRITERS LABORATORIES INC.

SANTA CLARA, CALIFORNIA LABORATORY

1655 Scott Boulevard, Santa Clara, CA 95050

Douglas Anderson Phone: 408-985-2400

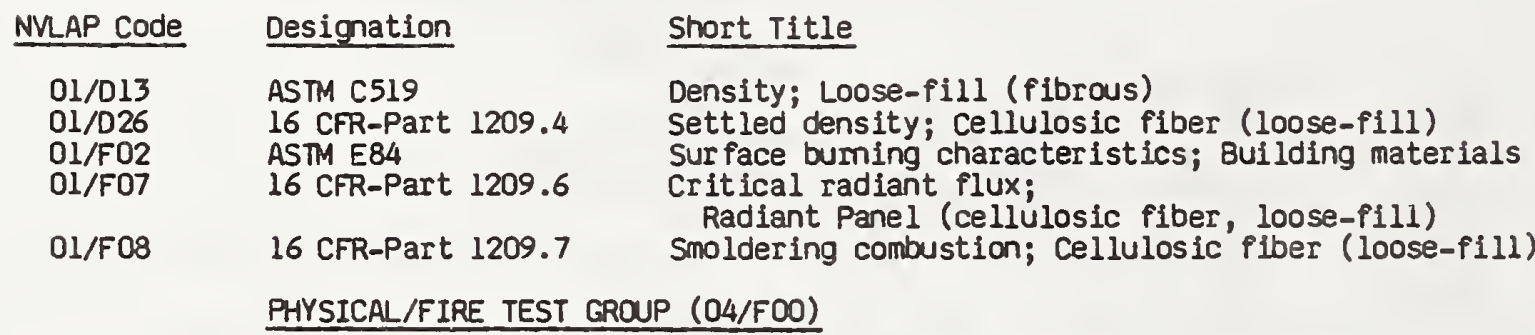 \\ NVLAP Code Designation}

Section of UL 737 Section of UL 1482

5 th Edition 2nd Edition

\begin{tabular}{|c|c|c|c|}
\hline NVLAP Code & Short Title & (November 9, 1982) & (January 24 \\
\hline $\begin{array}{l}\mathrm{O} / \mathrm{FO1} \\
\mathrm{O} / \mathrm{FO} 2\end{array}$ & $\begin{array}{l}\text { Test Installation } \\
\text { Temoerature Measurement }\end{array}$ & $\begin{array}{l}8 \\
9\end{array}$ & \\
\hline 04/FO4 & $\begin{array}{l}\text { Raperature Measulrement } \\
\text { Radiant Fire Test }\end{array}$ & 11 & 11 \\
\hline 04/F05 & Coal Fire Test & & 14 \\
\hline 04/F06 & Brand Fire Test & 12 & 12 \\
\hline 04/F07 & Flash Fire Test & 13 & \\
\hline 04/F08 & Strength Tests & 15 & \\
\hline 04/F09 & Stability Test & 16 & \\
\hline $04 / F 10$ & Glazing Test & 14 & \\
\hline
\end{tabular}

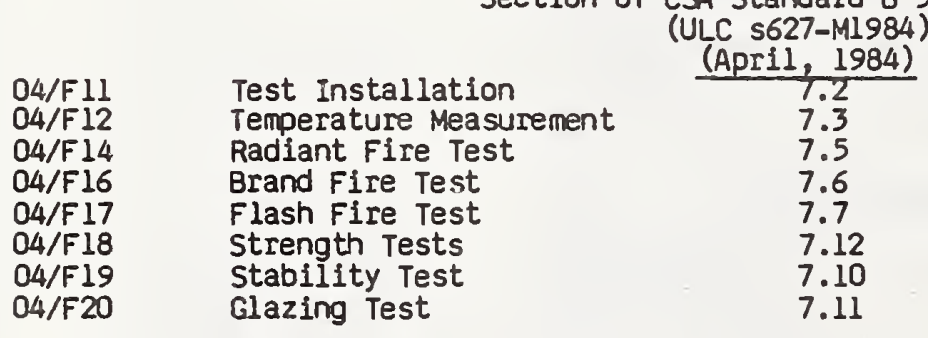

Section of CSA Standard B 366.2-M1984

MOBILE HOME TEST GROUP (04/MOO)

Section of UL 737 Section of UL 1482

5 th Edition 2 and Edition

NVLAP Code Short Title

(November 9, 1982) (January 24, 1983)

04/MO1

$04 / \mathrm{MO} 2$

Test Installation

$04 / \mathrm{MO3}$

Toxic Gas

Drop Test

17
17
17

17

17 
Section of CSA Standard B 366.2-M1984

(ULC S627-M1984)

(April, 1984)

$\begin{array}{lll}\text { 04/M04 } & \text { Test Installation } & 12 \\ \text { 04/M05 } & \text { Toxic Gas } & 12 \\ \text { 04/M06 } & \text { Drop Test } & 12\end{array}$

ELECTRICAL TEST GROUP (O4/EOO)

\begin{tabular}{|c|c|c|c|}
\hline NMLAP Code & Short Title & $\begin{array}{l}5 \text { th Edition } \\
\text { ovember 9, 1982) }\end{array}$ & $\begin{array}{l}\text { 2nd Edition } \\
\text { (January 24, 1983) }\end{array}$ \\
\hline O4/EO1 & Test Voltages & 33 & 33 \\
\hline O4/EO2 & $\begin{array}{l}\text { Temperature Measurements, } \\
\text { Electrical Components }\end{array}$ & 34 & 34 \\
\hline O4/EO3 & Input Test & 35 & 35 \\
\hline O4/EO4 & $\begin{array}{l}\text { Temperature Test, } \\
\text { Electrical Components }\end{array}$ & 36 & 36 \\
\hline O4/E05 & Leakage Current & 38 & 38 \\
\hline O4/E06 & Dielectric Withstand & 37 & 37 \\
\hline O4/E07 & Locked Rotor (stalled & 39 & 39 \\
\hline \multirow[t]{2}{*}{$04 / E C 8$} & $\begin{array}{l}\text { Motor) Temperature } \\
\text { Power cord Strain Relief }\end{array}$ & 40 & 40 \\
\hline & & $\begin{array}{r}\text { Section of CSA } \\
\text { C } 22.2 \text { NO. } 3 \\
1979 \\
\end{array}$ & $\begin{array}{c}\text { Section of CSA } \\
\mathrm{C} 22.2 \text { NO. } 113 \\
1982 \\
\end{array}$ \\
\hline O4/E09 & Temperature Measurements, & 6.4 & 6.2 \\
\hline 04/E10 & $\begin{array}{l}\text { Temperature Test, } \\
\text { Electrical Components }\end{array}$ & 6.4 & 6.2 \\
\hline $\begin{array}{l}\text { O4/E11 } \\
\text { O4/E12 } \\
\text { O4/E13 }\end{array}$ & $\begin{array}{l}\text { Leakage Current } \\
\text { Dielectric Withstand } \\
\text { Power Cord Strain Relief }\end{array}$ & $\begin{array}{l}6.8 \\
6.5 \\
6.9\end{array}$ & $\begin{array}{l}6.3 \\
6.3 \\
6.4\end{array}$ \\
\hline
\end{tabular}

NVLAP LAB CODE 0120

COMAERCIAL TESTING COMPANY

1215 South Hamilton Street, P.O. Box 985, Dalton, GA 30720 Jonathan Jackson Phone: 404-278-3935

Accreditation Renewal Date: January 1, 1988

\begin{tabular}{|c|c|c|}
\hline NVLAP Code & Designation & Short Title \\
\hline $01 / \mathrm{CO} 2$ & 16 CFR-Part 1209.5 & Corrosiveness; Cellulosic fiber (loose-fill) \\
\hline $01 / 026$ & 16 CFR-Part 1209.4 & Settled density; Cellulosic fiber (loose-fill) \\
\hline $01 / F 07$ & $\begin{array}{l}16 \text { CFr-Part } 1209.6 \\
\text { (formerly } H-I-515, P\end{array}$ & $\begin{array}{l}\text { Critical radiant flux; Radiant panel (cellulosic fiber) } \\
t \text { 4.8.7) }\end{array}$ \\
\hline $01 / F 08$ & $\begin{array}{l}16 \text { CFR-Part } 1209.7 \\
\text { (formerly HH-I-5i5, Par }\end{array}$ & $\begin{array}{l}\text { Smoldering combustion; Cellulosic fiber (loose-fill) } \\
t 4.8 .8 \text { ) }\end{array}$ \\
\hline $\begin{array}{l}\text { O1/TO6 } \\
03 / \mathrm{BO1} \\
03 / \mathrm{CO1} \\
03 / \mathrm{CO2} \\
03 / \mathrm{DO1}\end{array}$ & $\begin{array}{l}\text { ASTM C518 } \\
\text { UMA4A (Tabe 5) } \\
\text { AATCC } 16 E \\
\text { AATCC } 8 \\
\text { ASTM } 0418\end{array}$ & $\begin{array}{l}\text { Thermal transnission properties; Heat flow meter } \\
\text { Attached Cushion Tests } \\
\text { Color fastness to Light (Xenon Arc) } \\
\text { Color fastness to Crocking } \\
\text { Pile Yam Floor Covering Construction } \\
\text { Pile Weight - Uncoated (Section 8) } \\
\text { Pile Weight - Coated (Section 9) } \\
\text { Pile Thickness - (Sections } 10 \text { \& 11) } \\
\text { Tuft Height - (section 13) }\end{array}$ \\
\hline $\begin{array}{l}\text { O3/DO2 } \\
\text { 03/FO1 } \\
\text { 03/FO3 } \\
\text { O3/FO4 } \\
\text { 03/F05 } \\
\text { 03/SO1 }\end{array}$ & $\begin{array}{l}\text { DDD-C-95A } \\
\text { ASTM E } 84 \\
16 \text { CFR Part } 1630 \\
\text { ASTM E648 } \\
\text { ASTM E662 } \\
\text { ASTM D1335 }\end{array}$ & $\begin{array}{l}\text { Shrinkage } \\
\text { Surface Flammability } \\
\text { Methenamine Pill Test (FF-1-70) Sec. } 1630.4 \\
\text { Radiant Panel (Carpet) } \\
\text { Optical Density of Smoke Generated by Solid Materials } \\
\text { Tuft Bind of Floor Coverings }\end{array}$ \\
\hline
\end{tabular}


SPARRELL ENGINEERING RESEARCH CORPORATION

Bristol Road, P.0. Box 130, Damariscotta, ME 04543

James K. Sparrell Phone: 207-563-3224

Accreditation Renewal Date: January 1, 1988

\begin{tabular}{|c|c|}
\hline NVLAP Code & Designation \\
\hline Ol/TOL & ASTM Cl77 \\
\hline $\begin{array}{l}\text { O1/T04 } \\
\text { O1/T06 }\end{array}$ & $\begin{array}{l}\text { ASTM C236 } \\
\text { ASTM C518 }\end{array}$ \\
\hline
\end{tabular}

\title{
Short Title
}

Thermal transmission properties;

Low-temperature guarded hot plate

Thermal conductance; Guarded hot box

Thermal transmission properties; Heat flow meter

NVLAP LAB COOE 0122

TECHNICAL MICRONICS CONTROL, INC.

SCIENTIFIC SERVICES, INC

\section{P.0. Box 1330,210 Wym Drive, Huntsville, AL 35807}

Ms. Bharathi Ujjani Phone: 205-837-4430

Accreditation Renewal Date: April 1, 1987

\begin{tabular}{|c|c|c|}
\hline NVAP Code & Designation & Short Title \\
\hline $01 / \mathrm{CO} 2$ & 16 CFR-Part 1209.5 & Corrosiveness; Cellulosic fiber (loose-fill) \\
\hline $01 / 026$ & $\begin{array}{l}16 \text { CFR-Part } 1209.4 \\
\text { (formerly HH-I-5i5, par }\end{array}$ & $\begin{array}{l}\text { Settled density; Cellulosic fiber (loose-fill) } \\
\text { (4.8.1) }\end{array}$ \\
\hline $01 / F 07$ & $\begin{array}{l}16 \text { CFR-Part } 1209.6 \\
\text { (former ly HH-I-515, pa }\end{array}$ & $\begin{array}{l}\text { Critical radiant flux; Radiant panel Cellulosic fiber) } \\
\text { t } 4.8 .7 \text { ) }\end{array}$ \\
\hline $01 / F 08$ & $\begin{array}{l}16 \text { CFR-Part } 1209.7 \\
\text { (formerly } \mathrm{H}-\mathrm{I}-515 \text {, par }\end{array}$ & $\begin{array}{l}\text { Smoldering combustion; Cellulosic fiber (loose-fill) } \\
\text { 4.8.8) }\end{array}$ \\
\hline $\begin{array}{l}01 / T 06 \\
01 / V 05\end{array}$ & $\begin{array}{l}\text { ASTM C518 } \\
\text { ASTM C } 739(\mathrm{sec} .11) \\
\text { (formerly H-I-515, par }\end{array}$ & $\begin{array}{l}\text { Thermal transmission properties; Heat flow meter } \\
\text { Fungus; Cellulosic fiber (loose-fill) } \\
\text { t } 4.8 .6 \text { ) }\end{array}$ \\
\hline
\end{tabular}

NVLAP LAB COOE 0123

\author{
MANVILLE CORPORATION, $R$ \& D CENTER \\ P.O. Box 5108, Denver, CO 80217 \\ Joseph P. Ferraro Phone: 303-978-5553
}

Accreditation Renewal Date: January 1, 1988

\begin{tabular}{|c|c|c|}
\hline NULAP Code & Designation & Short Title \\
\hline $\begin{array}{l}01 / D 02 \\
01 / D 03\end{array}$ & $\begin{array}{l}\text { ASTM C167 } \\
\text { ASTM C } 209 \\
\text { (para. } 6 \text { in } 72 \text { version) }\end{array}$ & $\begin{array}{l}\text { Thickness and density; Blanket and batt } \\
\text { Thickness; Board (cellulosic fiber) }\end{array}$ \\
\hline $\begin{array}{l}01 / 004 \\
01 / 005\end{array}$ & $\begin{array}{l}\text { ASTM C209 } \\
\text { ASTM C209 } \\
\text { (para. } 13 \text { in } 72 \text { version) } \\
\text { by D1037 (para. 100-106 }\end{array}$ & $\begin{array}{l}\text { Water absorption, } 2 \text { hour; } \\
\text { Water absorption, } 24 \text { hour; Board (cellulosic fiber) } \\
6 \text { in } 78 \text { version) }\end{array}$ \\
\hline 01/D06 & $\begin{array}{l}\text { ASTM C209 } \\
\text { (para. } 14 \text { in } 72 \text { version) } \\
\text { by } 01037 \text { (para. 107-110 }\end{array}$ & $\begin{array}{l}\text { Linear expansion; Board (cellulosic fiber) } \\
\text { in } 72 \text { version) }\end{array}$ \\
\hline $\begin{array}{l}01 / 008 \\
01 / 009 \\
01 / 011\end{array}$ & $\begin{array}{l}\text { ASTM C302 } \\
\text { ASTM C303 } \\
\text { ASTM C } 356\end{array}$ & $\begin{array}{l}\text { Density; Preformed pipe insulation } \\
\text { Density; Preformed block insulation } \\
\text { Linear shrinkage; Soaking heat; } \\
\text { Preformed high temperature insulation }\end{array}$ \\
\hline $01 / 012$ & ASTM C411 & Hot-surface performance; High temperature insulation \\
\hline
\end{tabular}




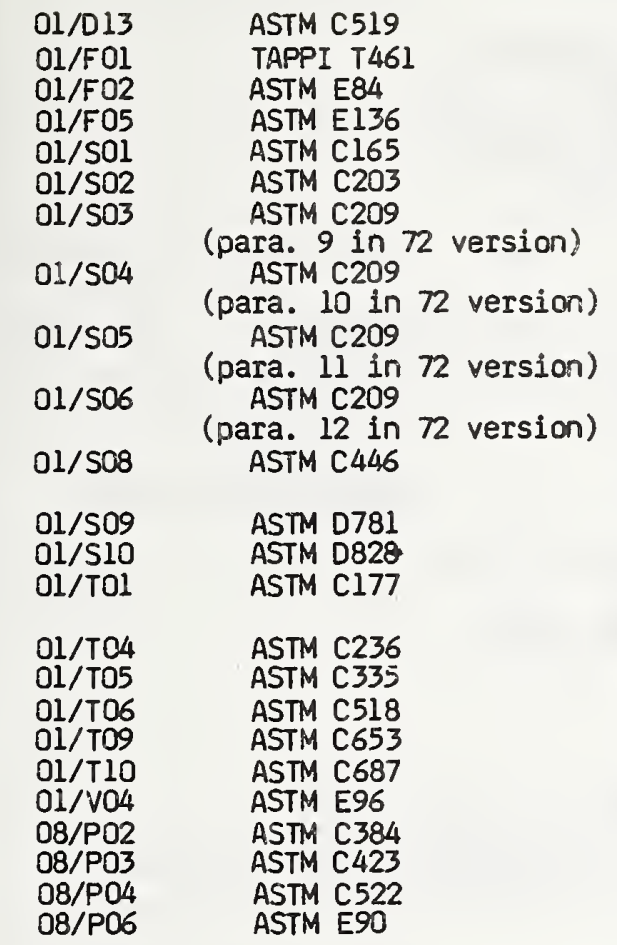

Density; Loose-fill (fibrous)

Flame Resistance; Paper and paperboard

Surface burning characteristics; Building materials

Behavior of Materials in a Vertical Tube Furnace

Conpressive properties; Thermal insulation (proc. A)

Breaking load/flexural strength; Preformed block insulation

Transverse strength; Board (cellulosic fiber)

Deflection at specified load;

Board (cellulosic fiber)

Tensile strength; Parallel to surface;

Board (cellulosic fiber)

Tensile strength; Perpendicular to surface

Breaking load/modulus of rupture;

Preformed pipe insulation

Puncture test; Paperboard and fiberboard

Tensile breaking strength; Paper and paperboard

Thermal transmission properties;

Low-temperature guarded hot plate

Thermal conductance; Guarded hot box

Thermal conductivity; Pipe insulation

Thermal transmission properties; Heat flow meter

Thermal resistance (Rec. Practice); Elanket (mineral fiber)

Thermal resistance (Rec. Practice); Loose-fill (fibrous)

Water vapor transmission; Thin sheets (proc. A)

Impedance and Absorption of Acoustical Materials

Sound Absorption and Sound Absorption coefficients

Airflow Resistance of Acoustical Materials

Airborne Sound Transmission Loss of Builoing Partitions

NVLAP LAB CODE 0124

\author{
OWENS-CORNING FIBERGLAS CORPORATION \\ PLANT LABORATORY \\ Box 89, 960 Central Expressway, Santa Clara, CA 95052 \\ J.P. Tetreault Phone: 408-727-3535
}

Accreditation Renewal Date: January 1, 1989

\begin{tabular}{|c|c|}
\hline NLAP Code & Designation \\
\hline $\begin{array}{l}01 / \mathrm{DO2} \\
01 / 009\end{array}$ & $\begin{array}{l}\text { ASTM Cl67 } \\
\text { ASTM C } 303\end{array}$ \\
\hline $01 / T 06$ & ASTM C518 \\
\hline
\end{tabular}

\section{Short Titie}

Thickness and density; Blanket and batt

Density; Preformed block insulation

Thermal transmission properties; Heat flow meter

\title{
NVLAP LAB CODE 0125
}

\author{
OWENS-CORNING FIBERGLAS CORPORATION \\ PLANT LABORATORY \\ 700 MCLaren Road, Fairburn, GA 30213 \\ C. J. Jackson Phone: 404-969-29:5
}

Accreditation Renewal Date: January 1, 1988

NVAP Code Designation

01/DO2 ASTM Cl67

OI/TO6 ASTM C518

\section{Short Title}

Thickness and density; Blanket and batt

Thermal transmission properties; Heat flow meter 


\section{OWENS-CORNING FIBERGLAS CORPORATION \\ PLANT LABORATORY \\ 300 Sunshine Road, Kansas City, KS 66115 \\ G.D. Growcock Phone: 913-281-2811}

Accreditation Renewal Date: January 1, 1988

$\begin{array}{cl}\text { NMLAP Code } & \text { Designation } \\ 01 / 002 & \text { ASTM C167 } \\ 01 / T 06 & \text { ASTM C } 518\end{array}$

\section{Short Title}

Thickness and density; Blanket and batt Thermal transmission properties; Heat flow meter

NVLAP LAB COOE 0128

OWENS-CORNING FIBERGLAS CORPCRATION

\author{
PLANT LABORATORY \\ P.0. Box 89, Delmar, NY 12054 \\ R.M. ROSSi Phone: 518-439-9341
}

Accreditation Renewal Date: January 1, 1988

\begin{tabular}{|c|c|}
\hline NVLAP Code & Designation \\
\hline $\begin{array}{l}\text { 01/002 } \\
01 / T 06\end{array}$ & $\begin{array}{l}\text { ASTM C167 } \\
\text { ASTM C518 }\end{array}$ \\
\hline
\end{tabular}

\title{
Short Title
}

Thickness and density; Blanket and batt Thermal transmission properties; Heat flow meter

NVLAP LAB CODE 0129

\author{
OWENS-CORNING FIBERGLAS CORPORATION \\ PLANT LABORATORY \\ Case Avenue, Newark, aH 43055 \\ P. D. Shull Phone: 614-345-3441
}

Accreditation Renewal Date: January 1, 1988

\begin{tabular}{|c|c|}
\hline NVLAP Code & Designation \\
\hline $\begin{array}{l}01 / 002 \\
01 / 009 \\
01 / T 06\end{array}$ & $\begin{array}{l}\text { ASTM C167 } \\
\text { ASTM C } 303 \\
\text { ASTM C518 }\end{array}$ \\
\hline
\end{tabular}

Short Title

Thickness and density; Blanket and batt Density; Preformed block insulation Thermal transmission properties; Heat flow meter

NMAP LAB COOE 0130

\author{
OWENS-CORNING FIEERGLAS CORPORATION \\ PLANT LABORATORY \\ P.0. Box 837, I-35 East, Waxahachie, TX 75165 \\ Mark Kwasowski Phone: 214-937-1340
}

Accreditation Renewal Date: January 1, 1988

NLAP Code Designation

01/002 ASTM Cl67

$01 / 009 \quad$ ASTM C 303

01/T06 ASTM C518

\section{Short Title}

Thickness and density; Blanket and batt Density; Preformed block insulation

Thermal transmission properties; Heat flow meter 
THE H. C. NUTTING COMPANY

4120 Airport Road, P.O. Box C, Cincinnati $\mathrm{OH} 45226$

Accreditation Renewal Date: January 1, 1988

$\begin{array}{lll}\text { NVLAP Code } & \text { Designation } & \text { Short Title } \\ \text { 02/G01 } & \text { ASTM C31 } & \text { Making and Curing Concrete Test Specimens in the field } \\ & \text { ASTM C172 } & \text { Sampling Freshly Mixed Concrete } \\ & \text { ASTM Cl43 } & \text { Slump of Portland Cement Concrete } \\ & \text { ASTM C138 } & \text { Unit veight, Yield, and Air Content (Gravimetric) } \\ & \text { ASTM C231 } & \text { Air Content of Freshly Mixed Concrete by the Pressure Method } \\ & \text { ASTM C173 } & \text { Air Content Volumetric Method } \\ 02 / A 01 & \text { ASTM C39 } & \text { Compressive Strength of Cylindrical Specimens }\end{array}$

NVLAP LAB CODE 0133

THE WALT KEELER COMPANY, INC.

826 East Lincoln Street, P.O. Box 197, Wichita, KS 67201

Kelly B. Callison Phone: 316-265-0615

Accreditation Renewal Date: January 1, 1988

\begin{tabular}{ll} 
NVLAP Code & Designation \\
\hline $02 / G 01$ & \\
& ASTM C31 \\
& ASTM C172 \\
& ASTM C143 \\
& ASTM C138 \\
& ASTM C231
\end{tabular}

Short Title

Making and Curing Concrete Test Specimens in the field

Sampling Freshly Mixed Concrete

Slump of Portland Cement Concrete

Unit Weight, Yield, and Air Content (Gravimetric)

Air Content of Freshly Mixed Concrete by the Pressure Method

NVLAP LAB CODE 0135

AGUIRRE ENGINEERS, INC.

13276 East Fremont Place, Englewood, CO 80112

Vukoslav E. Aguirre Phone: 303-799-8378

Accreditation Renewal Date: January 1, 1988

\begin{tabular}{|c|c|}
\hline IVLAP Code & Designation \\
\hline \multirow[t]{2}{*}{ 02/GOl } & $\begin{array}{l}\text { ASTM C } 31 \\
\text { ASTM C172 }\end{array}$ \\
\hline & $\begin{array}{l}\text { ASTM Cl43 } \\
\text { ASTM C138 } \\
\text { ASTM C } 231 \\
\text { ASTM C173 }\end{array}$ \\
\hline $\begin{array}{l}\text { O2/AO1 } \\
02 / A 02\end{array}$ & $\begin{array}{l}\text { ASTM C39 } \\
\text { ASTM C617 }\end{array}$ \\
\hline
\end{tabular}

Short Title

Making and Curing Concrete Test Specimens in the field

Sampling Freshly Mixed Concrete

Slump of Portland Cement Concrete

Unit Weight, Yield, and Air Content (Gravimetric)

Air Content of Freshly Mixed Concrete by the Pressure Method

Air Content Volumetric Method

Compressive Strength of Cylindrical Concrete Specimens

Capping Cylindrical specimens 
AMERICAN CARPET LABORATORIES, INC.

111 West Nashville Street, P.O. Box 357, Ringgold, GA 30736 Michael D. Connell Phone: 404-935-5672

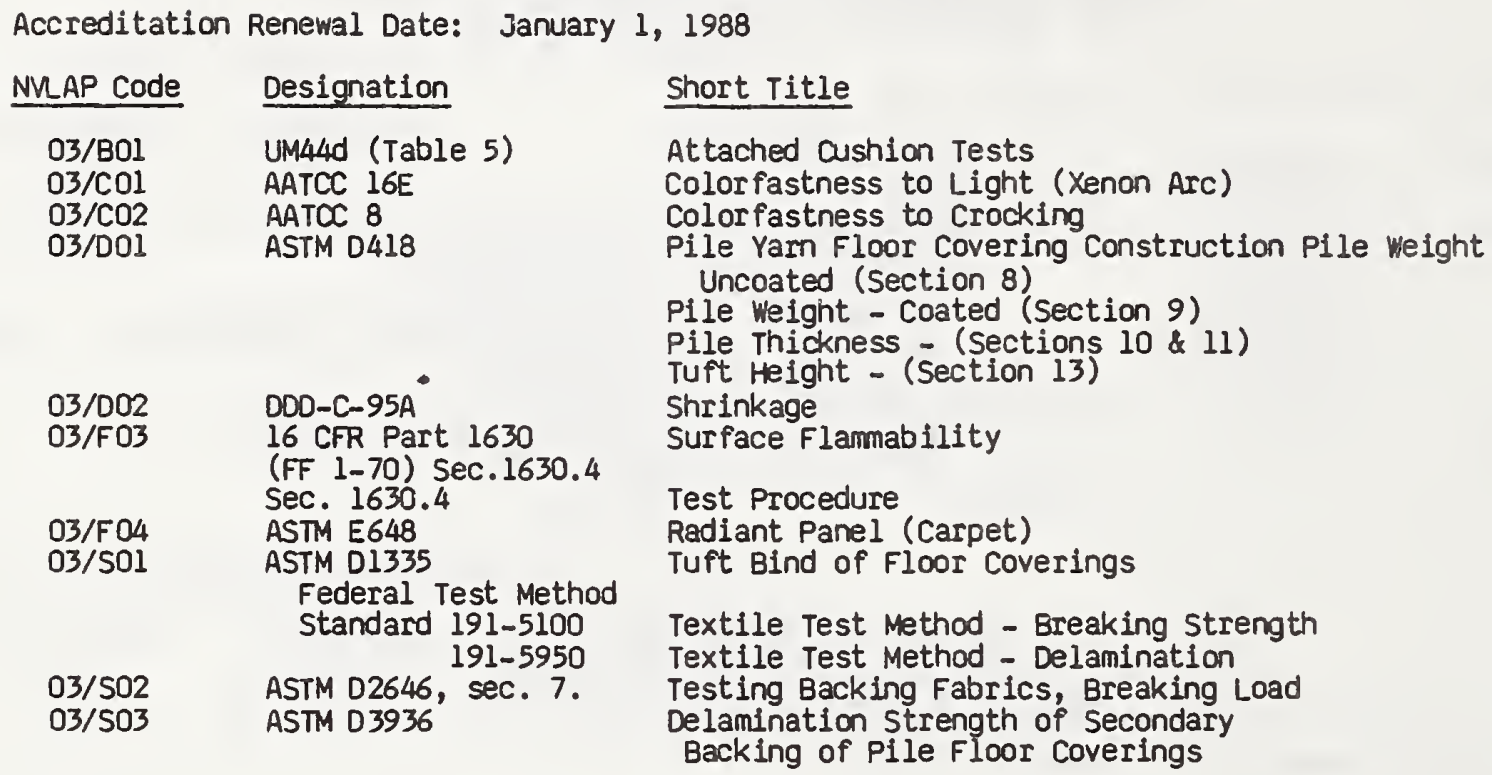

MLAP LAB COOE 0141

\author{
GENSTAR STONE PRODUCTS COMPANY \\ WHITE MARSH TECHNICAL CENTER \\ 10300 Pulaski Highway, White Marsh, MD 21162 \\ Roy K. Heaps Phone: 301-628-4064
}

Accreditation Renewal Date: January 1, 1988

\begin{tabular}{ll} 
NMLAP Code & Designation \\
\cline { 3 - 3 } $02 / \mathrm{GO} 1$ & \\
& ASTM C31 \\
& ASTM C172 \\
& ASTM C143 \\
& ASTM C138 \\
& ASTM C231 \\
& ASTM C173 \\
$02 / A O 1$ & ASTM C39 \\
$02 / A 02$ & ASTM C 617
\end{tabular}

\title{
Short Title
}

Making and Curing concrete Test Specimens in the field Sampling Freshly Mixed Concrete

Slump of Portland Cement Concrete

Unit Weight, Yield, and Air Content (Gravimetric)

Air Content of Freshly Mixed Concrete by the Pressure Method Air Content Volumetric Method

Compressive Strength of Cylindrical Concrete Specimens Capping Cylindrical Specimens

GEOSCIENCE LTD.

410 South Cedros Avenue, Solana Beach, CA 92075 Heinz F. Poppendiek Phone: 619-755-9396

Accreditation Renewal Date: January 1, 1988

\begin{tabular}{|c|c|}
\hline NVLAP Code & Designation \\
\hline $\begin{array}{l}01 / 008 \\
01 / F 05 \\
01 / T 01\end{array}$ & $\begin{array}{l}\text { ASTM C302 } \\
\text { ASTM E136 } \\
\text { ASTM C177 }\end{array}$ \\
\hline O1/T04 & ASTM C236 \\
\hline
\end{tabular}

Short Title

Density; Preformed pipe insulation Behavior of Materials in a Vertical Tube Furnace Thermal transmission properties; Low-temperature guarded hot plate Thermal conductance; Guarded hot box 
AMERICAN TESTING LABORATORIES, INC.

Box 4014, 784 Flory Mill Road, Lancaster, PA 17604 Jotn S. Kassees Phone: 717-569-0488

Accreditation Renewal Date: April 1, 1987

\begin{tabular}{|c|c|}
\hline NVLAP Code & Designation \\
\hline $02 / G 01$ & $\begin{array}{l}\text { ASTM C C31 } \\
\text { ASTM C172 } \\
\text { ASTM C143 } \\
\text { ASTM C138 } \\
\text { ASTM C231 } \\
\text { ASTM C173 }\end{array}$ \\
\hline 02/A01 & ASTM C39 \\
\hline
\end{tabular}

\section{Short Title}

Making and Curing Test Specimens

Sampling Freshly Mixed concrete

Slump of Portland Cement Concrete

Unit Weight, Yield, and Air content

Air Content-Pressure Method

Content-Volumetric Method

Compressive Strength of Cylindrical Specimens

NVAP LAB CODE 0149

$E$ \& B CARPET MILLS

P.O. BoX 2047, Dalton, GA 30720

Robert H. Davis Phone: 404-272-7783

Accreditation Renewal Date: January 1, 1988

\begin{tabular}{|c|c|}
\hline NVLAP Code & Designation \\
\hline $\begin{array}{l}03 / \mathrm{CO} 1 \\
03 / \mathrm{CO} 2 \\
03 / 001\end{array}$ & $\begin{array}{l}\text { AATCC } 16 \mathrm{E} \\
\text { AATCC } 8 \\
\text { ASTM D418 }\end{array}$ \\
\hline $\begin{array}{l}\text { 03/DO2 } \\
\text { 03/F03 }\end{array}$ & $\begin{array}{l}\text { DDD-C-95A } \\
16 \text { CFR Part } 1630 \\
(F F-1-70) \text { SeC. } 1630.4\end{array}$ \\
\hline $\begin{array}{l}03 / \mathrm{SO} 1 \\
03 / \mathrm{SO2} \\
03 / \mathrm{SO3}\end{array}$ & $\begin{array}{l}\text { ASTM D1335, } \\
\text { ASTM D2646, sec. } 7 \text {. } \\
\text { ASTM D } 3936\end{array}$ \\
\hline
\end{tabular}

Short Title

Colorfastness to Light (Xenon Arc)

Color fastness to Crocking

Pile Yarn Floor covering Construction

Pile Weight - Uncoated (Section 8)

Pile Weight - Coated (Section 9)

Pile Thickness - (Sections 10 \& 11)

Tuft Height - (Section 13)

Shrinkage

Methenamine Pill Test

Tuft Bind of Pile Floor Coverings

Testing Backing Fabrics, Breaking Load

Delamination Strength of Secondary Backing

of Pile Floor Coverings

NVAP LAB CODE 0151

HARDWOOD PLWWOD MANUFACTURERS ASSOCIATION

1825 Michael Faraday Drive, Reston, VA 22090

Gary Gramp Phone: 703-435-2900

Accreditation Renewal Date: January 1, 1988

NMAP Code Designation

03/F01 ASTM E84

03/F04 ASTM E648
Short Title

Surface Flammability

Radiant Panel (Carpet) 
THE ARUNDEL CORPORATION

GREENSPRING LABORATORY

6806 Greenspring Avenue, Baltimore, MD 21209

M. Joann Pet11lo Phone: 301-484-6022

Accreditation Renewal Date: January 1, 1988

\begin{tabular}{|c|c|}
\hline NUAP Code & Designation \\
\hline $02 / \mathrm{GO} 1$ & $\begin{array}{l}\text { ASTM C C 1 } \\
\text { ASTM C172 } \\
\text { ASTM C143 } \\
\text { ASTM C138 } \\
\text { ASTM C } 231 \\
\text { ASTM C173 }\end{array}$ \\
\hline $\begin{array}{l}\text { 02/AO1 } \\
02 / A 02\end{array}$ & $\begin{array}{l}\text { ASTM C39 } \\
\text { ASTM C } 617\end{array}$ \\
\hline
\end{tabular}

Short Title

Making and Curing concrete Test Specimens in the field

Sampling Freshly Mixed Concrete

Slump of Portland Cement Concrete

Unit Weight, Yield, and Air Content (Gravimetric)

Air Content of Freshly Mixed Concrete by the Pressure Method Air Content volumetric Method

Compressive Strength of Cylindrical Concrete Specimens Capping Cylindrical Specimens

\section{NLAP LAB COOE 0156}

BIGELOW-SANFORD, INC.

GEORGIA PUG MILL

Lyerly Street, Summerville, GA 30747

van A. Pullen Phone: 404-857-2421

\begin{tabular}{|c|c|c|}
\hline NUAP Code & Designation & Short Title \\
\hline $\begin{array}{l}03 / \mathrm{BO} 1 \\
03 / \mathrm{CO} 1 \\
03 / \mathrm{CO} 2 \\
03 / 001\end{array}$ & $\begin{array}{l}\text { UM 44d (Table 5) } \\
\text { AATCC } 16 E \\
\text { AATCC } 8 \\
\text { ASTM D } 418\end{array}$ & $\begin{array}{l}\text { Attached Qushion Tests } \\
\text { Colorfastness to Light (Xenon Arc) } \\
\text { Colorfastness to Crocking } \\
\text { Pile Yam Floor Covering Construction } \\
\text { Pile Weight - Uncoated (Section 8) } \\
\text { Pile Weight - Coated (Section 9) } \\
\text { Pile Thickness - (Sections 10 \& 11) } \\
\text { Tuft Height - (Section 13) }\end{array}$ \\
\hline $\begin{array}{l}\text { 03/D02 } \\
03 / F 03\end{array}$ & $\begin{array}{l}\text { DDD-C-95A } \\
16 \text { CFR Part } 1630 \\
(F F-1-70) \text { Sec. } 1630.4\end{array}$ & $\begin{array}{l}\text { Shrinkage } \\
\text { Methenamine Pill Test }\end{array}$ \\
\hline $\begin{array}{l}03 / 501 \\
03 / 503\end{array}$ & $\begin{array}{l}\text { ASTM D } 1335 \\
\text { ASTM D } 3936\end{array}$ & $\begin{array}{l}\text { Tuft Bind of Floor Coverings } \\
\text { Delamination Strength of Secondary } \\
\text { Backing of Pile Floor Coverings }\end{array}$ \\
\hline
\end{tabular}

NVAP LAB COOE 0160

CHISHOLM TRAIL TESTING AND ENGINEERING COMPANY, INC.

302 South Miller Street, Decatur, TX 76234

James F. Rosendahl Phone: 817-627-5216

Accreditation Renewal Date: January 1, 1988

\begin{tabular}{cl} 
NUAP Code & Designation \\
\hline $03 / C 01$ & AATCC 16E \\
$03 / C 02$ & AATCC 8 \\
$03 / D 01$ & ASTM D418
\end{tabular}

Short Title

Colorfastness to Light (Xenon Arc)

Colorfastness to Crocking

Woven and Tufted Pile Floor Covering

Pile Weight - Uncoated (Section 8)

pile Weight - Coated (Section 9)

Pile Thickness - (Sections 10 \& 11)

Tuft Height - (Section 13) 


$\begin{array}{ll}03 / D 02 & \text { DDD-C-95A } \\ 03 / F 03 & 16 \text { CFR Part 1630 } \\ & \text { (FF }-1-70 \text { ) Sec. } 1630.4 \\ 03 / \text { SO1 } & \text { ASTM D1335 } \\ 03 / \text { SO2 } & \text { ASTM D2646, sec. } 7 . \\ 03 / \text { SO3 } & \text { ASTM D3936 }\end{array}$

Shrinkage

Methenamine Pill Test

Tuft Bind of Pile Floor Coverings

Testing Backing Fabrics, Breaking Load

Delamination Strength of Secondary Backing of Pile Floor Coverings

NVLAP LAB CODE 0161

ENGINEERING TESTING LABORATORY CITY OF AKRON

1420 Triplett Blvd, Bldg \#2, Akron, OH 44306

Pawan K. Khaitan Phone: 216-375-2740

Accreditation Renewal Date: July 1, 1987

\begin{tabular}{|c|c|}
\hline MLAP Code & Designation \\
\hline 02/GOl & $\begin{array}{l}\text { ASTM C } 31 \\
\text { ASTM C C172 } \\
\text { ASTM Cl43 } \\
\text { ASTM C138 } \\
\text { ASTM C } 231 \\
\text { ASTM C173 }\end{array}$ \\
\hline
\end{tabular}

Short Title

Making and Curing Test specimens

Sampling Freshly Mixed Concrete

Slump of Portland cernent Concrete

Unit Weight, Yield, and Air Content

Air content-Pressure Method

02/AO1 ASTM C39

Content-Volumetric Method

Compressive Strength of cylindrical specimens

NVLAP LABCODE 0163

GALAXY CARPET MILLS, INC.

GALAXY TESTING LABORATORY

P.0. Box 800, Industrial Blvd., Chatsworth, GA 30705

3

Lou Childers Phone: 404-695-9611

Accreditation Renewal Date: January 1, 1988

\begin{tabular}{|c|c|}
\hline NVAP Code & Designation \\
\hline $\begin{array}{l}03 / \mathrm{CO} 1 \\
03 / \mathrm{CO2} \\
03 / 001\end{array}$ & $\begin{array}{l}\text { AATCC 16E } \\
\text { AATCC } 8 \\
\text { ASTM } 0418\end{array}$ \\
\hline $\begin{array}{l}\text { 03/D02 } \\
03 / F 03\end{array}$ & $\begin{array}{l}\text { DOD-C-95A } \\
16 \text { CFR Part } 1630 \\
\text { (FF } 1-70 \text { ) SeC. } 1630.4\end{array}$ \\
\hline $03 / 501$ & $\begin{array}{l}\text { ASTM D1335 } \\
\text { Federal Test Method } \\
\text { Standard } 191-5100 \\
191-5950\end{array}$ \\
\hline $\begin{array}{l}03 / \mathrm{SO2} \\
03 / 503\end{array}$ & $\begin{array}{l}\text { ASTM D2646, sec. } 7 . \\
\text { ASTM D } 3936\end{array}$ \\
\hline
\end{tabular}

\section{Short Title}

Color fastness to Light (Xenon Arc)

Color fastness to Crocking

Plle Yarn Floor Covering Construction

Pile Weight - Uncoated (Section 8)

Pile Weight - Coated (Section 9)

Pile Thickness - (Sections 10 \& 11)

Tuft Height - (Section 13)

Shrinkage

Surface Flammability

Test Procedure

Tuft Bind of Floor Coverings

Textile Test Method - Breaking Strength

Textile Test Method - Delamination

Testing Backing Fabrics, Breaking Load

Delamination Strength of Secondary

Backing of Pile Floor Coverings 
INDEPENDENT TEXTILE TESTING SERVICE, INC.

P.0. Box 1948, 1503 Murray Avenue, Dalton, GA 30722 Harry M. Fry Phone: 404-278-3013

Accreditation Renewal Date: January 1, 1988

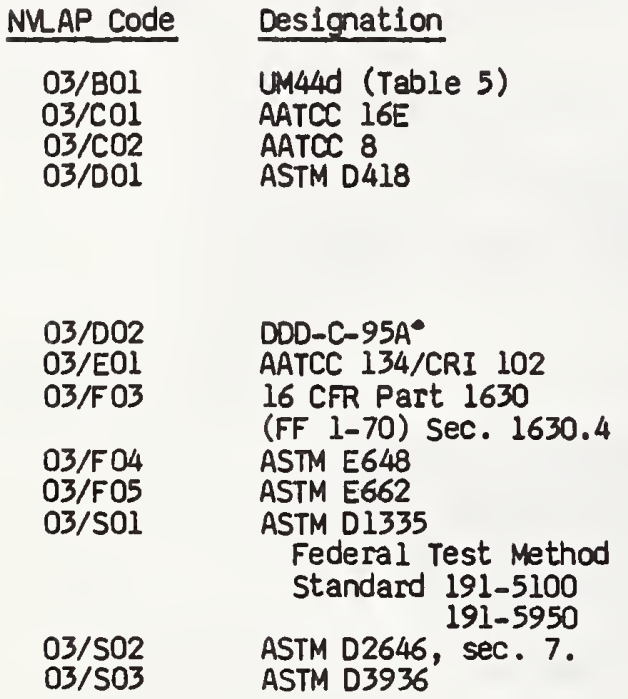

Short Title

Attached Cushion Tests

Colorfastness to Light (Xenon Arc)

Colorfastness to Crocking

plie Yam floor covering construction

Pile Weight - Uncoated (Section 8)

Pile Weight - coated (section 9)

Pile Thickness - (Sections 10 \& 11 )

Tuft Height - (Section 13)

Shrinkage

Electrostatic Propensity of Carpets

Surface Flamability

Test Procedure

Radiant Panel (Carpet)

Optical Density of Smoke Generated by Solld Materials

Tuft Bind of Floor Coverings

Textile Test Method - Breaking Strength

Textile Test Method - Delamination

Testing Backing Fabrics, Breaking Load

Delamination Strength of Secondary

Backing of Pile Floor coverings

NMAP LAB COOE 0175

DOW CHEMICAL U.S.A

NORTH HAVEN LABORATORIES

410 Sackett Point Road, P.0. Box 430, North Haven, CT 06473

Herbert G. Nadeau Phone: 203-281-2762

Accreditation Renewal Date: October 1, 1987

\begin{tabular}{|c|c|}
\hline NYLAP Code & Designation \\
\hline $01 / 021$ & ASTM D2126 \\
\hline $01 / 028$ & ASTM D2126 \\
\hline 01/T06" & ASTM C518 \\
\hline
\end{tabular}

Short Title

Response to thermal and humid aging (proc. E); Rigid cellular plastics

Response to thermal and humid aging

(proc. G); Rigid cellular plastics

Thermal transmission properties; Heat flow meter

NYLAP LAB CODE 0176

W. R. GRACE \& COMPANY

CONSTRUCTION PRODUCTS DIVISION

62 Whittemore Avenue, Cambridge; MA 02140

Michael Dallaire Phone: 617-876-1400

Accreditation Renewal Date: January 1, 1988

\begin{tabular}{|c|c|}
\hline NLLAP Code & Designation \\
\hline $02 / \mathrm{GOl}$ & $\begin{array}{l}\text { ASTM C C 1 } \\
\text { ASTM C172 } \\
\text { ASTM C143 } \\
\text { ASTM C1138 } \\
\text { ASTM C C231 } \\
\text { ASTM C173 }\end{array}$ \\
\hline
\end{tabular}

Short Title

Making and Curing Concrete Test Specimens in the field

Sampling Freshly Mixed Concrete

slump of Portland Cement Concrete

Unit Weight, Yield, and Air Content (Gravimetric)

Air Content of Freshly Mixed Concrete by the Pressure Method

Air Content volumetric Method

02/A01 ASTM C39

Compressive Strength of Cylindrical Concrete Specimens 


\section{ATLANTIC TESTING LABORATORIES, LIMITED \\ CICERO DIVISION \\ P.0. Box 356, Route 31 at Route 81, Cicero, NY 13039 \\ Robert van der Horst Phone: 315-699-5281}

Accreditation Renewal Date: April 1, 1987

\begin{tabular}{cl} 
NMAP Code & Designation \\
\hline $02 / G 01$ & \\
& ASTM C31 \\
& ASTM C172 \\
& ASTM C143 \\
& ASTM C138 \\
& ASTM C231 \\
& ASTM C173 \\
02/AO1 & ASTM C39
\end{tabular}

Short Title

Making and Curing Test Specimens

Sampling Freshly Mixed concrete

Slump of Portland cement Concrete

Unit Weight, Yield, and Air Content

Air Content-Pressure Method

Content-Volumetric Method

Compressive Strength of Cylindrical Specimens

NVLAP LAB CODE 0178

BIGELOW-SANFORD, INC.

TECHNICAL SERVICES

P.0. Box 3089, Greenville, SC 29602

Hamir D. Merchant Phone: $803-299-2630$

Accreditation Renewal Date: January 1, 1988

NLAP Code Designation

03/801

$03 / \mathrm{COI}$

$03 / \mathrm{CO} 2$

03/D01

$\begin{array}{ll}\text { 03/DO2 } & \text { DOD-C-95A } \\ 03 / E 01 & \text { AATCC 134/CRI 102 } \\ 03 / F 03 & \text { 16 CFR Part } 1630 \\ \text { 03/F04 } & \text { (FF-1-70) SeC.1630.4 } \\ \text { ASTM E648 } \\ \text { 03/F05 } & \text { ASTM E662 } \\ 03 / S 01 & \text { ASTM D1335 } \\ 03 / 502 & \text { ASTM D2646, SEC. 7. } \\ 03 / S 03 & \text { ASTM D3936 }\end{array}$

Short Title

Attached Cushion Tests

Color fastness to Light (Xenon Arc)

color fastness to Crocking

Pile Yarn floor Covering construction

Plle Weight - Uncoated (Section 8)

Pile Weight - Coated (Section 9)

pile Thickness - (Sections 10 \& 11)

Tuft Height - (Section 13)

Shrinkage

Electrostatic Propensity of Carpets

Methenamine Pill Test

Test Procedure

Radiant Panel (Carpet)

Optical Density of Smoke Generated by Solid Materials

Tuft Bind of floor Coverings

Testing Backing Fabrics, Breaking Load

Delamination Strength of Secondary

Backing of Plle Floor coverings

NVLAP LAB CODE 0183

A \& HFLOOD ENGINEERING DIVISION, P.S.I., INC. 4421 Harrison Street, Hillside, IL 60162 Char les Siegert Phone: 312-449-0500

Accreditation Renewal Date: April 1, 1987

\begin{tabular}{|c|c|}
\hline NLAP Code & Designatio \\
\hline $02 / \mathrm{GO}$ & $\begin{array}{l}\text { ASTM C C31 } \\
\text { ASTM C1772 } \\
\text { ASTM C143 } \\
\text { ASTM CI38 } \\
\text { ASTM C231 } \\
\text { ASTM C173 }\end{array}$ \\
\hline
\end{tabular}

02/AO1

ASTM C39
Short Title

Making and Curing Test Specimens

Sampling Freshly Mixed concrete

Slump of Portland Cement concrete

Unit Weight, Yield, and Air content

Air Content-Pressure Method

Content-Volumetric Method

Compressive Strength of Cylindrical Specimens 
TWIN CITY TESTING AND ENGINEERING LABORATORY, INC.

662 Cromwell Avenue, St. Paul, MN 55114

Richard Stehly Phone: 612-645-3601

Accreditation Renewal Date: January 1, 1988

\begin{tabular}{|c|c|}
\hline NVLAP Code & Designation \\
\hline $\begin{array}{l}01 / \mathrm{TO4}^{2} \\
\text { 02/G01 }\end{array}$ & $\begin{array}{l}\text { ASTM C236 } \\
\text { ASTM C } 31 \\
\text { ASTM C C172 } \\
\text { ASTM C143 } \\
\text { ASTM C138 } \\
\text { ASTM C } 231\end{array}$ \\
\hline & ASTM C173 \\
\hline $\begin{array}{l}\text { O2/AO1 } \\
\text { O2/AO2 }\end{array}$ & $\begin{array}{l}\text { ASTM C } 39 \\
\text { ASTM C617 }\end{array}$ \\
\hline
\end{tabular}

Short Title

Thermal conductance; Guarded hot box

Making and Curing Concrete Test Specimens in the field

Sampling Freshly Mixed Concrete

Slump of Portland Cement Concrete

Unit Weight, Yield, and Air Content (Gravimetric)

Air Content of Freshly Mixed Concrete by the Pressure Method

Air content Volumetric Method

Compressive Strength of Cylindrical Concrete Specimens Capping Cylindrical Specimens

NVLAP LAB CODE 0190

CORONET CARPETS

CORONET INDUSTRIES

P.0. Box 1248, Cleveland Drive, Dalton, GA 30720

Winfred $L$. Jones Phone: 404-259-4511

Accreditation Renewal Date: January 1, 1988

\begin{tabular}{|c|c|}
\hline NVLAP Code & Designation \\
\hline $\begin{array}{l}03 / \mathrm{CO} 1 \\
03 / \mathrm{CO} 2 \\
03 / 001\end{array}$ & $\begin{array}{l}\text { AATCC } 16 E \\
\text { AATCC } 8 \\
\text { ASTM D418 }\end{array}$ \\
\hline $\begin{array}{l}\text { 03/D02 } \\
03 / \mathrm{SO1}\end{array}$ & $\begin{array}{l}\text { DDD-C-95A } \\
\text { ASTM D } 1335 \\
\text { Federal Test Method } \\
\text { Standard } 191-5100 \\
191-5950\end{array}$ \\
\hline $\begin{array}{l}03 / \mathrm{SO2} \\
03 / \mathrm{SO3}\end{array}$ & $\begin{array}{l}\text { ASTM D2646, sec. } 7 \\
\text { ASTM D3936, }\end{array}$ \\
\hline
\end{tabular}

Short Title

Colorfastness to Light (Xenon Arc)

Color fastness to Crocking

Pile Yarn Floor Covering Construction

Pile Weight - Uncoated (Section 8)

Pile Weight - Coated (Section 9)

Pile Thickness - (Sections 10 \& 11)

Tuft Height - (Section 13)

Shrinkage

Tuft Bind of Floor Coverings

Textile Test Method - Breaking Strength

Textile Test Method - Delamination

Testing Backing Fabrics, Breaking Load

Delamination Strength of Secondary

Backing of Pile Floor Coverings

NMAP LAB CODE 0191

STS CONSULTANTS, LTD.

111 Pfingsten Road, Northbrook, IL 60062

Ronald E. Hutchens Phone: 312-272-6520

Accreditation Renewal Date: January 1, 1988

\begin{tabular}{|c|c|}
\hline NVLAP Code & Designation \\
\hline $02 / 601$ & $\begin{array}{l}\text { ASTM C C 1 } \\
\text { ASTM C172 } \\
\text { ASTM C143 } \\
\text { ASTM C C138 } \\
\text { ASTM C } 231 \\
\text { ASTM C } 173\end{array}$ \\
\hline $\begin{array}{l}\text { 02/A01 } \\
02 / A 02\end{array}$ & $\begin{array}{l}\text { ASTM C39 } \\
\text { ASTM C617 }\end{array}$ \\
\hline
\end{tabular}

Short Title

Making and curing Concrete Test Specimens in the field Sampling Freshly Mixed Concrete

Slump of Portland Cement Concrete

Unit Weight, Yield, and Air Content (Gravimetric)

Air Content of Freshly Mixed Concrete by the Pressure Method

Air content Volumetric Method

Compressive Strength of Cylindrical concrete Specimens

Capping Cylindrical Specimens 
SMITH-EMERY COMPANY

781 East Hashington Boulevard, Los Angeles, CA 90021 George E. Battey, Jr. Phone: 213-749-3411

Accreditation Renewal Date: January 1, 1988

\begin{tabular}{|c|c|}
\hline NYLAP Code & Designation \\
\hline $\begin{array}{l}\text { CONCRETE } \\
02 / \mathrm{GOI}\end{array}$ & $\begin{array}{l}\text { ASTM C } 31 \\
\text { ASTM C } 172 \\
\text { ASTM C } 143 \\
\text { ASTM C } 138 \\
\text { ASTM C } 231\end{array}$ \\
\hline $\begin{array}{l}\text { O2/AO1 } \\
\text { O2/AO2 }\end{array}$ & $\begin{array}{l}\text { ASTM C173 } \\
\text { ASTM C } 39 \\
\text { ASTM C } 617\end{array}$ \\
\hline $\begin{array}{c}\text { AGSREGATES } \\
\text { O2/A03 } \\
02 / A 04 \\
\text { O2/A06 }\end{array}$ & $\begin{array}{l}\text { ASTM C29 } \\
\text { ASTM C } 340 \\
\text { ASTM C } 88\end{array}$ \\
\hline O2/A07 & ASTM Cl17 \\
\hline $\begin{array}{l}\text { 02/A08 } \\
02 / A 09 \\
02 / A 10 \\
\text { O2/A11 }\end{array}$ & $\begin{array}{l}\text { ASTM Cl23 } \\
\text { ASTM Cl27 } \\
\text { ASTM Cl28 } \\
\text { ASTM Cl31 }\end{array}$ \\
\hline $\begin{array}{l}02 / A 12 \\
02 / A 13\end{array}$ & $\begin{array}{l}\text { ASTM Cl36 } \\
\text { ASTM Cl42 }\end{array}$ \\
\hline
\end{tabular}

Short Title

Making and Curing Concrete Test Specimens in the field Sampling Freshly Mixed Concrete

Slump of Portland Cement Concrete

Unit Weight, Yield, and Air Content (Gravimetric)

Air content of Freshly Mixed Concrete by the Pressure Method

Air Content volumetric Method

Compressive Strength of Cylindrical Concrete Specimens

Capping Cylindrical Specimens

Unit Weight and Voids in Aggregates

Organic Impurities in Fine Aggregate

Soundness of Aggregates by Use of

Sodium Sulfate or Magnesium Sulfate

Materials Finer than 75- m (No. 200) Sieve

in Mineral Aggregates by Washing

Lightweight Pieces in Aggregate

Specific Gravity and Absorption of coarse Aggregate

Specific Gravity and Asbortion of Fine Aggregate

Resistance to Degradation of Small-Size Coarse

Aggregates in the Los Angeles Machine

Sieve Analysis of Fine and Coarse Aggregates

Clay Lumps and Friable Particles in Aggregates

NYLAP LAB CODE 0193

SHAW INDUSTRIES, INC.

Plant \#4, S. Hamilton St. Ext., P.O. Drawer 2128, Dalton, GA 30720

Dernis Hart Phone: 404-278-3812

Accreditation Renewal Date: January 1, 1988

\begin{tabular}{cl} 
NUAP Code & Designation \\
\hline $03 / C O 1$ & AATCC $16 E$ \\
$03 / C 02$ & AATCC 8 \\
$03 / D 01$ & ASTM D418
\end{tabular}

03/F03 16 CFR Part 1630

(FF-1-70) Sec.1630.4

03/SO1 ASTM D1335

03/SO2 ASTM D2646, Sec. 7

$03 / 503$
Short Title

Colorfastness to Light (Xenon ArC)

Colorfastness to Crocking

Pile Yarn Floor covering Construction

Pile Weight - Uncoated (Section 8)

Pile Weight - Coated (Section 9)

Pile Thickness - (Sections 10 \& 11)

Tuft Height - (Section 13)

Methenamine Pill Test

Tuft Bind of Floor Coverings

Testing Backing Fabrics, Breaking Load

Delamination strength of Secondary

Backing of Pile Floor coverings 
GARCO TESTING LABORATORIES

532 West 3560 South, Salt Lake City, UT 84107

Douglas L. Watson Phone: 801-266-4498

Accreditation Renewal Date: January 1, 1988

\begin{tabular}{|c|c|}
\hline NMLAP Code & Designation \\
\hline $02 / G 01$ & $\begin{array}{l}\text { ASTM C C 1 } \\
\text { ASTM C C } 172 \\
\text { ASTM C143 } \\
\text { ASTM C } 138 \\
\text { ASTM C231 }\end{array}$ \\
\hline $\begin{array}{l}\text { O2/AO1 } \\
\text { O2/A02 }\end{array}$ & $\begin{array}{l}\text { ASTM C173 } \\
\text { ASTM C } 39 . \\
\text { ASTM C } 617\end{array}$ \\
\hline
\end{tabular}

Short Title

Making and curing concrete Test Specimens in the field Sampling Freshly Mixed Concrete

Slump of Portland Cement Concrete

Unit Weight, Yield, and Air Content (Gravimetric)

Air Content of Freshly Mixed Concrete by the Pressure Method

Air content Volumetric Method

Compressive Strength of Cylindrical concrete specimens

Capping Cylindrical Specimens

TEXAS TESTING LABORATORIES, INC.

1526 South Good-Latimer Expressway, P.O. Box 2144, Dallas, TX 75221

George H. Pluto Phone: 214-428-7481

Accreditation Renewal Date: January 1, 1988

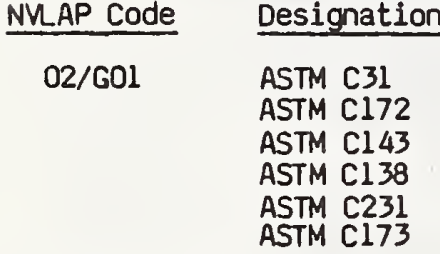

02/AO1 ASTM C39

\section{Short Title}

Making and Curing Concrete Test Specimens in the field Sampling Freshly Mixed Concrete

Slump of Portland Cement Concrete

Unit Weight, Yield, and Air content (Gravimetric)

Air Content of Freshly Mixed Concrete by the Pressure Method Air content volumetr ic Method

Compressive Strength of Cylindrical Specimens

NVAP LAB CODE 0197

\author{
WORLD CARPETS \\ QUALITY CONTROL PHYSICAL TESTING \\ one World Plaza, Dalton, GA 30720 \\ Wayne Murdock Phone: 404-278-8000
}

Accreditation Renewal Date: January 1, 1988

\begin{tabular}{|c|c|c|}
\hline NVLAP Code & Designation & Short Title \\
\hline $\begin{array}{l}03 / \mathrm{CO} 1 \\
03 / \mathrm{CO2} \\
03 / 001\end{array}$ & $\begin{array}{l}\text { AATCC } 16 E \\
\text { AATCC } 8 \\
\text { ASTM D } 418\end{array}$ & $\begin{array}{l}\text { Colorfastness to Light (Xenon Arc) } \\
\text { Color fastness to Crocking } \\
\text { Pile Yam Floor Covering Construction } \\
\text { Pile Weight - Uncoated (Section 8) } \\
\text { Pile Weight - Coated (Section 9) } \\
\text { Pile Thickness - (Sections 10 \& 11) } \\
\text { Tuft Height - (Section 13) }\end{array}$ \\
\hline $03 / F 03$ & $\begin{array}{l}16 \text { CFR Part } 1630 \\
\text { (FF } 1-70) \text { Sec. } 1630.4\end{array}$ & $\begin{array}{l}\text { Surface Flammability } \\
\text { Test Procedure }\end{array}$ \\
\hline $\begin{array}{l}03 / \mathrm{SO} \\
03 / \mathrm{SO2} \\
03 / \mathrm{SO}\end{array}$ & $\begin{array}{l}\text { ASTM D } 1335 \\
\text { ASTM D2646, sec. } 7 \\
\text { ASTM D } 3936\end{array}$ & $\begin{array}{l}\text { Tuft Bind of Floor Coverings } \\
\text { Testing Backing Fabrics, Breaking Load } \\
\text { Delamination Strength of Secondary } \\
\text { Backing of Pile Floor Coverings }\end{array}$ \\
\hline
\end{tabular}


PTL-INSPECTORATE INC.

850 Poplar Street, Pittsburgh, PA 15220

william $H$. Levelius Phone: 412-922-4000

Accreditation Renewal Date: October 1, 1987

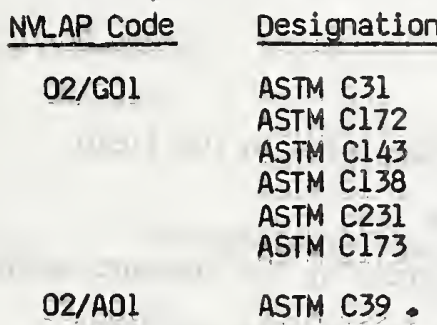

Short Title

Making and curing Test Specimens

Sampling Freshly Mixed Concrete

Slump of Portland Cement Concrete

Unit Weight, Yield, and Air content

Air Content-Pressure Method

Content-Volumetr ic Method

Compressive Strength of Cylindrical Specimens

NVLAP LAB CODE 0203

\section{CALMAT CO.}

CONCRETE AND AGGREGATES LABORATORY

16009 Foothill Blvd." "Irwindale, CA 91706

James Neal Van Nest Phone: 818-334-0304

Accreditation Renewal Date: January 1, 1988

\begin{tabular}{|c|c|}
\hline NMAP Code & Designation \\
\hline $02 / \mathrm{GO}$ & $\begin{array}{l}\text { ASTM C C31 } \\
\text { ASTM C172 } \\
\text { ASTM C143 } \\
\text { ASTM C138 } \\
\text { ASTM C231 } \\
\text { ASTM C173 }\end{array}$ \\
\hline $\begin{array}{l}02 / A 01 \\
02 / A 02\end{array}$ & $\begin{array}{l}\text { ASTM C39 } \\
\text { ASTM C } 617\end{array}$ \\
\hline
\end{tabular}

\section{Short Title}

Making and curing Test Specimens

Sampling Freshly Mixed concrete

Slump of Portland Cement Concrete

Unit Weight, Yield, and Air content

Air Content-Pressure Method

content-Volumetric Method

Compressive Strength of Cylindrical Specimens

Capping Cylindrical specimens

NRAP LAB CODE 0206

R. W. SIDLEY, INC.

QUALITY CONTROL LABORATORY

6900 Madison Road, Thompson, OH 44086

James R. Cannizzaro Phone: 216-298-3232

Accreditation Renewal Date: January 1, 1988

NMAP Code Designation

$02 / 601$

$02 / \mathrm{AO} 2$
ASTM C 31

ASTM $\mathrm{Cl} 72$

ASTM C143

ASTM C138

ASTM C231

ASTM C173

ASTM C39

ASTM $\mathrm{C} 617$

\section{Short Title}

Making and curing Concrete Test Specimens in the field Sampling Freshly Mixed concrete

Slump of Portland Cement Concrete

Unit Height, Yield, and Air content (Gravimetric)

Air Content of Freshly Mixed Concrete by the Pressure Method

Air Content Volumetric Method

Compressive Strength of Cylindrical concrete Specimens

Capping cylindrical specimens 
GULF COAST TESTING LABORATORY, INC.

1205 North Tancahua Street, Corpus Christi, TX 78401

Doyne Reyrolds Phone: 512-882-5411

Accreditation Renewal Date: January 1, 1988

NVAP Code Designation

CONCRETE

$02 / G 01$

O2/A02

AGGREGATES

O2/A03
$02 / A 04$
$02 / A 05$
O2/A06
O2/A07
O2/A08
02/A09
O2/A10
O2/A11
O2/A12
O2/A13
02/A16

ASTM C 31

ASTM $\mathrm{Cl} 172$

ASTM C143

ASTM C138

ASTM C231

ASTM $\mathrm{Cl} 73$

ASTM C 39

ASTM C617

ASTM C29

ASTM C 40

ASTM C87

ASTM C88

ASTM C1L7

ASTM $\mathrm{C} 123$

ASTM C127

ASTM C128

ASTM C131

ASTM C136

ASTM C142

ASTM 02419
Short Title

Making and curing Concrete Test Specimens in the field Sampling Freshly-Mixed Concrete

Slump of Portland Cement Concrete

Unit Weight, Yield, and Air Content (Gravimetric)

Air content of Freshly Mixed Concrete by the Pressure Method Air Content Volumetric Method

Compressive Strength of Cylindrical Concrete Specimens

Capping cylindrical Specimens

Unit Weight and Voids in Aggregates

Organic Impurities in Fine Aggregate

Effect of Organic Impurities in Fine

Aggregates on Strength of , Mortar

Soundess of Aggregates by Use of Sodium

Sulfate or Magnesium Sulfate

Materials Finer than 75- $m$ (No . 200) Sieve

in Mineral Aggregates by Washing

Lightweight Pleces in Aggregate

Specific Gravity and Absorption of Coarse Aggregate

Specific Gravity and Absorption of Fine Aggregate

Resistance to Degradation of Small-Size

Coarse Aggregates in the Los Angeles Machine

Sieve Analysis of Fine and Coarse Aggregates

Clay Lumps and Friable Particles in Aggregates

Sand Equivalent Value of Soils and Fine Aggregate

NVLAP LAB COOE 0210

INSTA-FOAM PRODUCTS, INC.

1500 Cedarwood Drive, Joliet, IL 60435

Greg Luegering Phone: 815-741-6819

Accreditation Renewal Date: January 1, 1988

\begin{tabular}{lll} 
NVAP Code & & Designation \\
\hline $01 / 015$ & & ASTM D756 \\
$01 / D 16$ & & ASTM D756 \\
$01 / D 17$ & & ASTM D756 \\
$01 / D 18$ & & ASTM D1622 \\
$01 / D 20$ & ASTM D2126 \\
$01 / D 22$ & ASTM D2126 \\
$01 / 023$ & ASTM D2842 \\
$01 / D 27$ & ASTM D2126 \\
$01 / D 28$ & ASTM D2126 \\
$01 / S 11$ & ASTM D1621 \\
$01 / T 06$ & ASTM C518 \\
$01 / V 04$ & ASTM E96
\end{tabular}

Short Title

Weight and shape changes; Accelerated service (proc. A); Plastics

Weight and shape changes; Accelerated service (proc. B); Plastics

Weight and shape changes; Accelerated service (proc. E); Plastics

Apparent density; RIgid cellular plastics

Response to thermal and humid aging (proc. D); Rigid cellular plastics

Response to thermal and humid aging (proc. F); Rigid cellular plastics

Hater absorption; Rigid cellular plastics

Besponse to thermal and humld aging (proc. C); RIgtot cetlular plastics

Response to thermal and humid aging (proc. G); Rigid cellular plastics

Compressive properties; Rigid cellular plastics (proc. A-Crosshead)

Thermal transmission propertles; Heat flow meter Water vapor transmission; Thin sheets (proc. A) 
CONSTRUCTION MATERIALS CONSULTANTS, INC.

1000 West Fillmore street, Colorado Springs, CD 80907 Ivan A. Vanaken Phone: 303-632-2588

Accreditation Renewal Date: July 1, 1987

\begin{tabular}{|c|c|}
\hline NYLAP code & Designation \\
\hline $02 / \mathrm{GO}$ & $\begin{array}{l}\text { ASTM C } C 1 \\
\text { ASTM C } 172 \\
\text { ASTM Cl } \\
\text { ASTM C133 } \\
\text { ASTM C } 231 \\
\text { ASTM Cl73 }\end{array}$ \\
\hline O2/AOL & ASTM C39. \\
\hline
\end{tabular}

Short Title

Making and curing Test Specimens Sampling Freshly Mixed Concrete Slump of Portland Cement Concrete Unit Weight, Yield, and Air content Air Content-Pressure Method Content-Volumetric Method

Compressive strength of Cylindrical Specimens

NVLAP LAB CODE 0216

USG CORPORATION

700 North Highway 45, Libertyville, IL 60048 William F. Porter Phone: 312-362-9797

Accreditation Renewal Date: July 1, 1987

$\frac{\text { NMAP Code }}{01 / T 06} \quad \frac{\text { Designation }}{\text { ASTM C518 }}$

Short Title

01/T06 ASTM C518

Thermal transmission properties; Heat flow meter

NVLAP LAB COOE 0218

APACHE BUILDING PRODUCTS COMPANY

2025 East Linden Avenue, Linden, NJ 07036

Demis W. Rosato Phone: 201-486-6723

Accreditation Renewal Date: October 1, 1987

\begin{tabular}{|c|c|}
\hline NVLAP Code & Designation \\
\hline $\begin{array}{l}01 / 018 \\
01 / 021\end{array}$ & $\begin{array}{l}\text { ASTM D1622 } \\
\text { ASTM D2126 }\end{array}$ \\
\hline $01 / 027$ & ASTM D2126 \\
\hline $01 / 511$ & ASTM D1621 \\
\hline 01/T06 & ASTM C518 \\
\hline
\end{tabular}

Short Title

Apparent density; Rigid cellular plastics

Response to thermal and humid aging (proc. E); Rigid cellular plastics

Response to thermal and humid aging (proc. c); Rigid cellular plastics

Compressive properties; Rigid cellular plastics (proc. A-Crosshead)

Thermal transmission properties; Heat flow meter

NVLAP LAB CODE 0220

STRATTON LABORATORIES

Highway 61, South, P.O. Box 1007, Cartersville, GA 30120 Jack R. Kilgore Phone: 404-382-9350

Accreditation Renewal Date: October 1, 1987

\begin{tabular}{|c|c|}
\hline NYLAP Code & Designation \\
\hline $03 / F 03$ & 16 CFR Part 1630 \\
\hline $\begin{array}{l}03 / F 04 \\
03 / 501\end{array}$ & $\begin{array}{l}\text { AFE } 1 \text {-79 } 564 \text { SeC. } 1630 \\
\text { ASTM D1335 }\end{array}$ \\
\hline $\begin{array}{l}03 / 502 \\
03 / 503\end{array}$ & $\begin{array}{l}\text { ASTM D2646, sec. } 7 \\
\text { ASTM D } 3936\end{array}$ \\
\hline
\end{tabular}

Short Title

Sur face F lammability

Test Procedure

Radiant Panel (Carpet)

Tuft Bind of Floor Coverings

Testing Backing Fabrics, Breaking Load

Delamination Strength of Secondary Backing of

Pile Floor Coverings 
SALEM CARPET LABORATORY

P.0. Box 10, Chatsworth, GA 30720

Michael A. Corbin Phone: 404-935-2241

\begin{tabular}{|c|c|}
\hline NVLAP Code & Designation \\
\hline $\begin{array}{l}03 / \mathrm{CO} 1 \\
03 / \mathrm{CO} 2 \\
03 / 001\end{array}$ & $\begin{array}{l}\text { AATCC } 16 E \\
\text { AATCC } 8 \\
\text { ASTM D } 418\end{array}$ \\
\hline $\begin{array}{l}\text { 03/D02 } \\
03 / F 03\end{array}$ & $\begin{array}{l}\text { DOD-C-95A } \\
16 \text { CFR Part } 1630 \\
(F F-1-70) \text { Sec. } 1630.4\end{array}$ \\
\hline $\begin{array}{l}03 / F 04 \\
03 / \mathrm{SO}^{2} \\
03 / \mathrm{SO}^{2} \\
03 / \mathrm{SO3}^{2}\end{array}$ & $\begin{array}{l}\text { ASTM E648 } \\
\text { ASTM D1335 } \\
\text { ASTM D2646, sec. } 7 \\
\text { ASTM D } 3936\end{array}$ \\
\hline
\end{tabular}

Short Title

Color fastness to Light (Xenon Arc)

Colorfastness to Crocking

Pile Yarn Floor Covering Construction

Pile Weight - Uncoated (Section 8)

Pile Weight - Coated (Section 9)

Pile Thickness - (Sections 10 \& 11)

Tuft Height - (Section 13)

Shrinkage

Surface Flammability

Test Procedure

Radiant Panel (Carpet)

Tuft Bind of Floor Coverings

Testing Backing Fabrics, Breaking Load

Delamination Strength of Secondary Backing of

Pile Floor Coverings

NVLAP LAB COOE 0223

PFS CORPORATION

2402 Daniels Street, Madison, WI 53704

Ed Starostovic Phone: 608-221-3361

Accreditation Renewal Date: January 1, 1988

\begin{tabular}{|c|c|c|c|}
\hline IMLAP Code & Short Title & $\begin{array}{c}\text { Section of UL } 737 \\
\text { 5th Edition } \\
\text { (March 1, 1982) }\end{array}$ & $\begin{array}{c}\text { Section of UL } 1482 \\
\text { 2nd Edition } \\
\text { (January 24, 1983) } \\
\end{array}$ \\
\hline & \multicolumn{3}{|c|}{ PHYSICAL/FIRE TEST GROUP (04/F00) } \\
\hline $\begin{array}{l}\text { O4/FO1 } \\
04 / F 02 \\
04 / F 04 \\
04 / F 05 \\
04 / F 06 \\
04 / F 07 \\
04 / F 08 \\
04 / F 09 \\
04 / F 10\end{array}$ & $\begin{array}{l}\text { Test Installation } \\
\text { Temperature Measurement } \\
\text { Radiant Fire Test } \\
\text { Coal Fire Test } \\
\text { Brand Fire Test } \\
\text { Flash Fire Test } \\
\text { Strength Tests } \\
\text { Stability Test } \\
\text { Glazing Test }\end{array}$ & $\begin{array}{l}8 \\
9 \\
11 \\
12 \\
13 \\
15 \\
16 \\
14\end{array}$ & $\begin{array}{l}8 \\
9 \\
11 \\
14 \\
12 \\
13 \\
16 \\
16 \\
15\end{array}$ \\
\hline
\end{tabular}

MOBILE HOME TEST GROUP (04/MOO)

04/MOI

$04 / \mathrm{MO} 2$

$04 / \mathrm{MO3}$

Test Installation

Toxic Gas

Drop Test

$17 \quad 17$

ELECTRICAL TEST GROUP (04/EOO)

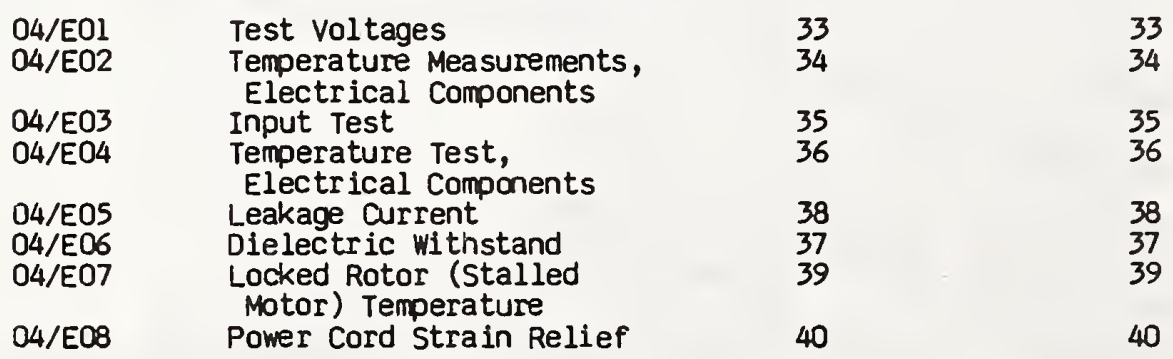


ARNOLD GREENE TESTING LABORATORIES

A DIVISION OF CONAM INSPECTION

2 Millbury Street, Auburn, MA 01501

Robert J. Halliday Phone: 617-235-7330

Accreditation Renewal Date: January 1, 1988

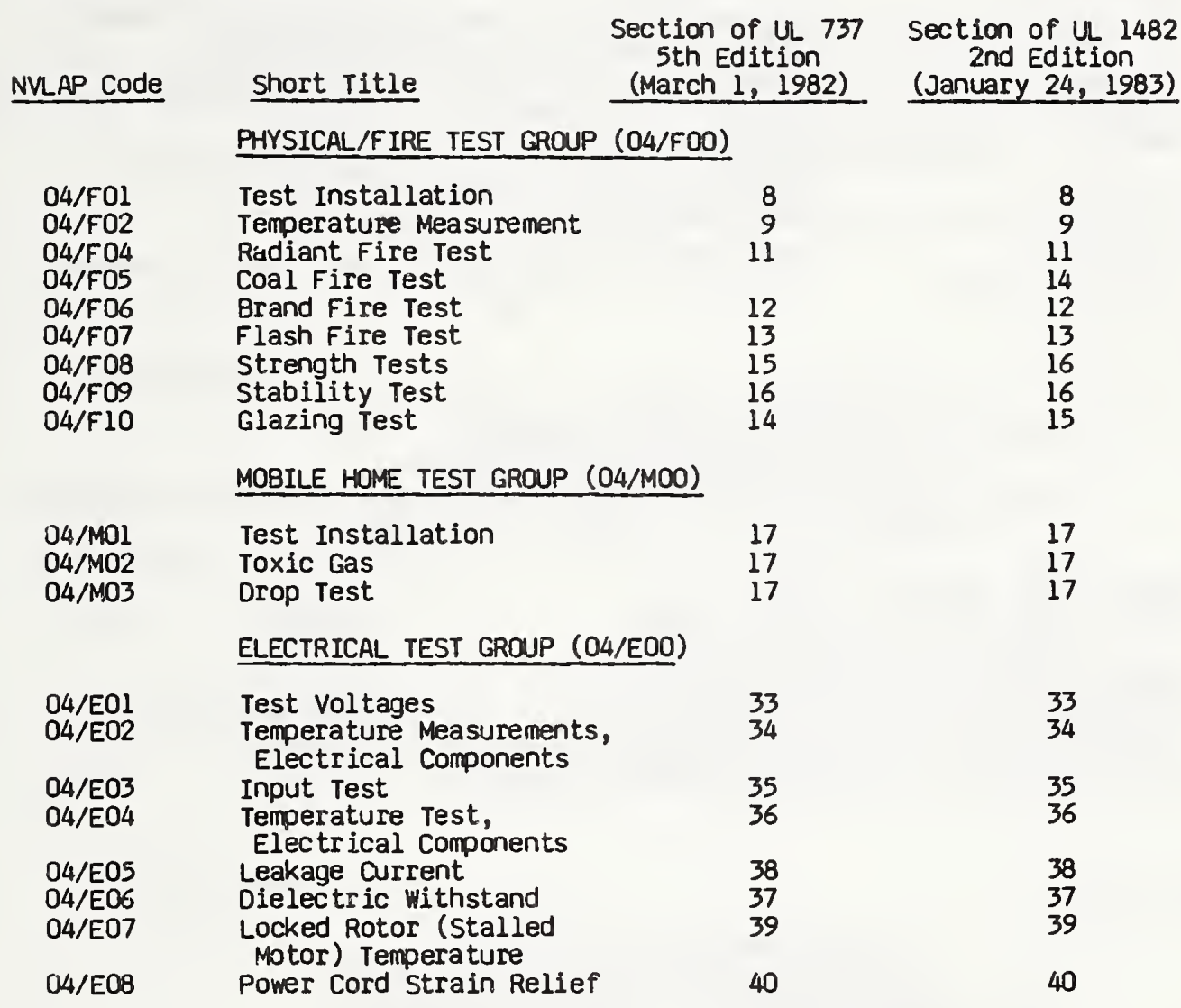

NVLAP LAB COOE 0226

WISS, JANNEY, ELSTNER ASSOCIATES, INC.

330 Pfingsten Road, Northbrook, IL 60062

Jerry G. Stockbridge Phone: 312-272-7400

Accreditation Renewal Date: July 1, 1987

NVAP Code Designation

$01 / T_{04} \quad$ ASTM C236
Short Title

Thermal conductance; Guarded hot box 


\section{RIVERBANK ACOUSTICAL LABORATORIES \\ P.0.Box 189, 1512 Batavia Avenue, Geneva, IL 60134 \\ John W. Kopec Phone: 312-232-0104}

Accreditation Renewal Date: April 1, 1987

\begin{tabular}{|c|c|}
\hline NLAP Code & Designation \\
\hline $\begin{array}{l}\text { 08/P03 } \\
\text { 08/P05 } \\
\text { 08/P06 } \\
\text { 08/PO7 } \\
\text { 08/P10 }\end{array}$ & $\begin{array}{l}\text { ASTM C423 } \\
\text { ASTM C523 } \\
\text { ASTM E90 } \\
\text { ASTM E492 } \\
\text { ANSI S1.31 }\end{array}$ \\
\hline 08/P17 & ISO 3741 \\
\hline O8/EO1 & $\begin{array}{l}\text { NNSI B71.1 } \\
\text { (para. } 9 \text { and 21) }\end{array}$ \\
\hline
\end{tabular}

Short Title

Sound Absorption and Sound Absorption Coefficients Light Reflectance of Acoustical Materials

Airborne Sound Transmission Loss of Building Partitions Impact Sound Transmission Through Floor-Ceiling Assemblies Sound Power Levels, Broad-Band Nolse Sources in Reverberation Rooms (100-10,000 Hz) Sound Power Levels, Broad-Band Sources in Reverberation Rooms $(100-10,000 \mathrm{~Hz})$ Sound Level Tests; Power Lawn Mowers, Lawn and Garden Tractors and Lawn Tractors

NVLAP LAB CODE 0228

ARMSTRONG WORLD INOUSTRIES TECHNICAL CENTER, ACOUSTICS LABORATORY

2500 Columbia Avenue, P.0.Box 3511, Lancaster, PA 17604 William R. Reed Phone: 717-396-5523

Accreditation Renewal Date: January 1, 1988

NMAP Code Designation

08/P03 ASTM C423

OB/PO7 ANSI/ASTM E492

\section{Short Title}

Sound Absorption and Sound Absorption Coefficients Impact Sound Transmission Through

Floor-ceiling Assemblies

NVLAP LAB CODE 0229

GOLD BOND DILOING PRODUCTS

A NATIONAL GYPSUM DIVISION, RESEARCH CENTER

1650 Military Road, Buffalo, NY 14217

Terry Williamson Phone: 716-873-9750

Accreditation Renewal Date: April 1, 1987

\begin{tabular}{cll} 
NMLAP Code & & Designation \\
\cline { 1 - 2 } 08/P03 & & ASTM C423 \\
08/P06 & ASTM E90 \\
08/E21 & AMA-1-II
\end{tabular}

Short Title

Sound Absorption and Sound Absorption coefficients Airborne sound Transmission Loss of Building Partitions ceiling Sound Transmission Test by Two-Room Method 
VIRGINIA CONCRETE LABORATORY

6555 Industrial Drive, P.0. Box 666, Springfield, VA 22150

Donald Blevins Phone: 703-354-7100

Accreditation Renewal Date: April 1, 1987

\begin{tabular}{cll} 
NMAP Code & Designation & Short Title \\
\cline { 1 - 1 } $02 /$ GO1 & ASTM C31 & Making and Curing Test Specimens \\
& ASTM C172 & Sampling Freshly Mixed Concrete \\
& ASTM C143 & Slump of Port land Cement Concrete \\
& ASTM C138 & Unit Weight, Yield, and Air Content \\
& ASTM C231 & Air Content-Pressure Method \\
ASTM C173 & Content-Volumetric Method \\
02/AO1 & ASTM C39. & Compressive Strength of Cylindrical Specimens
\end{tabular}

NVLAP LAB COOE 0232

RITCHIE LABORATORIES

1820 North Mosley, P.O. Box 4048, Wichita, KS 67204

Donald J. Brockel Phone: 316-263-9937

Accreditation Renewal Date: January 1, 1988

\begin{tabular}{cll} 
NLAP Code & Designation & Short Title \\
\cline { 3 - 3 } $02 / G 01$ & ASTM C31 & Making and Curing Concrete Test Specimens in the field \\
& ASTM C172 & Sampling Freshly Mixed Concrete \\
& ASTM C143 & Slump of Port land Cement Concrete \\
& ASTM CI38 & Unit Weight, Yield, and Air Content (Gravimetric) \\
& ASTM C231 & Air Content of Freshly Mixed Concrete by the Pressure Method \\
02/AO1 & ASTM C173 & Air Content Volumetric Method \\
& ASTM C39 & Compressive Strength of Cylindrical Specimens
\end{tabular}

NVLAP LAB COOE 0233

STS CONSULTANTS, LTD.

FAIRFAX VA OFFICE

2929-C Eskridge Road, Fairfax, VA 22031

John M. Grusha Phone: 703-698-5300

Accreditation Renewal Date: October 1, 1987

$\begin{array}{ll}\text { NVLAP Code } & \text { Designation } \\ 02 / \text { GO1 } & \\ & \text { ASTM C31 } \\ & \text { ASTM C172 } \\ & \text { ASTM C143 } \\ & \text { ASTM C138 } \\ & \text { ASTM C231 } \\ & \text { ASTM C173 } \\ 02 / A 01 & \text { ASTM C39 }\end{array}$

Short Title

Making and Curing Test Specimens

Sampling Freshly Mixed Concrete

Slump of Port land Cement Concrete

Unit Weight, Yield, and Air content

Air Content-Pressure Method

content-Volumetric Method

O2/AO1 ASTM C39

Compressive Strength of Cylindrical Specimens 


\section{PITTSBURGH TESTING LABORATORY \\ SYRACUSE NY PLANT LABORATORY \\ 6159 East Mallory Road, Syracuse, NY 13057 \\ W.J. Peters Phone: 315-437-7043}

Accreditation Renewal Date: April 1, 1987

\begin{tabular}{|c|c|}
\hline NVLAP Code & Designation \\
\hline $02 / G 01$ & $\begin{array}{l}\text { ASTM C C } 1 \\
\text { ASTM C C } 172 \\
\text { ASTM Cl } 43 \\
\text { ASTM Cl38 } \\
\text { ASTM C } 231 \\
\text { ASTM Cl73 }\end{array}$ \\
\hline $02 / A 01$ & ASTM C39 \\
\hline
\end{tabular}

Short Title

Making and Curing Test Specimens Sampling Freshly Mixed Concrete Slump of Port land cement Concrete Unit Weight, Yield, and Air content Air Content-Pressure Method Content-Volumetric Method

Compressive Strength of Cylindrical specimens

NVLAP LAB CODE 0239

\author{
HUFCOR ACOUSTICAL LABORATORY \\ HOUGH MANUFACTURING CORP. \\ P.O. Box 591, 1205 Norwood Road, Janesville, WI 53547 \\ Stanley Kowalczyk Phone: 608-756-1241 \\ Accreditation Renewal Date: October 1, 1987

\begin{tabular}{|c|c|}
\hline NVLAP code & Designation \\
\hline $\begin{array}{l}\text { O8/P03 } \\
\text { 08/P06 }\end{array}$ & $\begin{array}{l}\text { ASTM C423 } \\
\text { ASTM E90 }\end{array}$ \\
\hline
\end{tabular} \\ Short Title \\ Sound Absorption and Sound Absorption Coefficients \\ Airborne Sound Transmission Loss of Building Partitions
}

NVLAP LAB CODE 0240

OMNI ENVIRONENTAL SERVICES, INC.

SOLID FUES TESTING LAB

10950 SW 5th Street, Suite 160, Beaverton, OR 97005

Raymond $w$. Downey Phone: 503-643-3755

Accreditation Renewal Date: January 1, 1988

PHYSICAL/FIRE TEST GROUP (04/FO0)

\begin{tabular}{|c|c|c|c|}
\hline NVLAP Code & Short ritle & $\begin{array}{c}5 \text { th Edition } \\
\text { (November } 9,1982 \text { ) } \\
\end{array}$ & $\begin{array}{l}\text { 2nd Edit } \\
\text { (January 24, }\end{array}$ \\
\hline $\begin{array}{l}\text { O4/FO1 } \\
04 / F 02 \\
04 / F 04 \\
04 / F 05 \\
04 / F 06 \\
04 / F 07 \\
04 / F 08 \\
04 / F 09 \\
04 / F 10\end{array}$ & $\begin{array}{l}\text { Test Installation } \\
\text { Temperature Measurement } \\
\text { Radiant Fire Test } \\
\text { Coal Fire Test } \\
\text { Brand Fire Test } \\
\text { Flash Fire Test } \\
\text { Strength Tests } \\
\text { Stability Test } \\
\text { Glazing Test }\end{array}$ & $\begin{array}{l}8 \\
9 \\
11 \\
12 \\
13 \\
15 \\
16 \\
14\end{array}$ & $\begin{array}{l}8 \\
9 \\
11 \\
14 \\
12 \\
13 \\
16 \\
16 \\
15\end{array}$ \\
\hline
\end{tabular}

Section of CSA Standard B 366.2-M1984

\begin{tabular}{c} 
(ULC s627-M1984) \\
(April, 1984) \\
\hline 7.2 \\
7.3 \\
7.5 \\
7.6 \\
7.7
\end{tabular}

04/F 11

$04 / F 12$

$04 / F 14$

$04 / F 16$

04/F 17

Test Installation

Temperature Measurement

Radiant Fire Test

Brand Fire Test

Flash Fire Test
Section of UL 737 Section of UL 1482

5th Edition
(November 9, 1982) $\quad \begin{gathered}\text { 2nd Edition } \\ \text { (January 24, 1983) }\end{gathered}$

16

16


$\begin{array}{lll}\text { 04/F18 } & \text { Strength Tests } & 7.12 \\ 04 / F 19 & \text { Stability Test } & 7.10\end{array}$

04/F20 Glazing Test 7.11

MOBILE HOME TEST GROUP (04/MOO)

NVLAP Code

Short Title

Section of UL 737

5 th Edition

04/MOL

$04 / \mathrm{MO2}$

$04 / \mathrm{MO3}$

Test Installation

Toxic Gas

Drop Test

$\begin{array}{ll}17 & 17 \\ 17 & 17 \\ 17 & 17\end{array}$

Section of CSA Standard B 366.2-M1984

(ULC s627-M1984)

(April, 1984)

$04 / \mathrm{MO}_{4}$

iest Installation

12

Toxic Gas

12

04/MO6

Drop Test

12

ELECTRICAL TEST GROUP (04/EOO)

\begin{tabular}{|c|c|c|c|}
\hline MLAP code & Short Title & $\begin{array}{l}\text { 5th Edition } \\
\text { Vovember 9, 1982) }\end{array}$ & $\begin{array}{l}\text { 2nd Edition } \\
\text { (January 24, 1983) }\end{array}$ \\
\hline $\begin{array}{l}\text { O4/EO1 } \\
\text { O4/EO2 }\end{array}$ & $\begin{array}{l}\text { Test Voltages } \\
\text { Temperature Measurements, } \\
\text { Electrical Components }\end{array}$ & $\begin{array}{l}33 \\
34\end{array}$ & $\begin{array}{l}33 \\
34\end{array}$ \\
\hline $\begin{array}{l}\text { O4/EO3 } \\
\text { O4/EO4 }\end{array}$ & $\begin{array}{l}\text { Input Test } \\
\text { Temperature Test, } \\
\text { Electrical Components }\end{array}$ & $\begin{array}{l}35 \\
36\end{array}$ & $\begin{array}{l}35 \\
36\end{array}$ \\
\hline $\begin{array}{l}\text { O4/EO5 } \\
\text { O4/EO6 } \\
\text { O4/EO7 }\end{array}$ & $\begin{array}{l}\text { Leakage ourrent } \\
\text { Oielectric Withstand } \\
\text { Locked Rotor (Stalled } \\
\text { Motor) Temperature }\end{array}$ & $\begin{array}{l}38 \\
37 \\
39\end{array}$ & $\begin{array}{l}38 \\
37 \\
39\end{array}$ \\
\hline \multirow[t]{2}{*}{ O4/E08 } & Power Cord Strain Rellef & 40 & 40 \\
\hline & & $\begin{array}{c}\text { Section of CSA } \\
22.2 \mathrm{No} .3 \\
1979\end{array}$ & $\begin{array}{l}\text { Section of CSA } \\
\text { C } 22.2 \text { No. } 113 \\
1982 \\
\end{array}$ \\
\hline O4/E09 & $\begin{array}{l}\text { Temperature Measurements, } \\
\text { Electrical Comoonents }\end{array}$ & 6.4 & 6.2 \\
\hline O4/E10 & Temperature Test, & 6.4 & 6.2 \\
\hline $\begin{array}{l}\text { O4/E11 } \\
\text { O4/E12 } \\
\text { O4/E13 }\end{array}$ & $\begin{array}{l}\text { Leakage Current } \\
\text { Dielectric withstand } \\
\text { Power Cord Strain Relief }\end{array}$ & $\begin{array}{l}6.8 \\
6.5 \\
6.9\end{array}$ & $\begin{array}{l}6.3 \\
6.3 \\
6.4\end{array}$ \\
\hline
\end{tabular}

NVLAP LAB CODE 0241

UNITED STATES TESTING COMPANY, INC. UNI TECH SERVICES GROUP-WESTERN DIVISION

3536 Dakdale Road, Modesto, CA 95355

Thomas Gaeto Phone: 209-527-2271

Accreditation Renewal Date: January 1, 1988

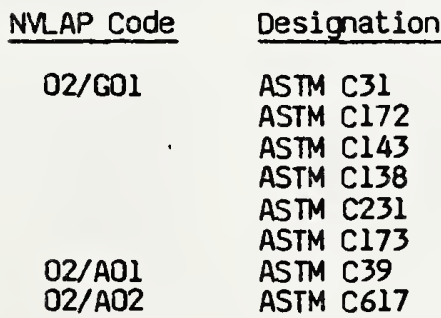

\section{Short Title}

Making and Curing Concrete Test Specimens in the field Sampling Freshly Mixed Concrete Slump of Port land Cement Concrete Unit Weight, Yield, and Air Content (Gravimetric) Air Content of Freshly Mixed Concrete by the Pressure Method Air Content Volumetric Method Compressive Strength of Cylindrical Concrete Specimens Capping Cylindrical specimens 
CUSTOM COATING, INC.

204 West Industrial Blvd., Dalton, GA 30720

David C. Robinson Phone: 404-277-3778

Accreditation Renewal Date: April 1, 1987

$\begin{array}{lll}\text { NVAP Code } & \text { Designation } & \text { Short Title } \\ 03 / F 03 & \begin{array}{ll}\text { 16 CFR Part 1630 } \\ \text { (FF 1-70) Sec. 1630.4 }\end{array} & \text { Surface Flammability } \\ & \text { Test Procedure }\end{array}$

MVAP LAB COOE 0244

NORTHNEST TESTING LABORATORIES, INC.

5405 N. Lagoon Avenue, P.O. Box 17126, Portland, OR 97217-0126 Harry L. Lippy Phone: 503-289-1778

Accreditation Renewal Date: January 1, 1988

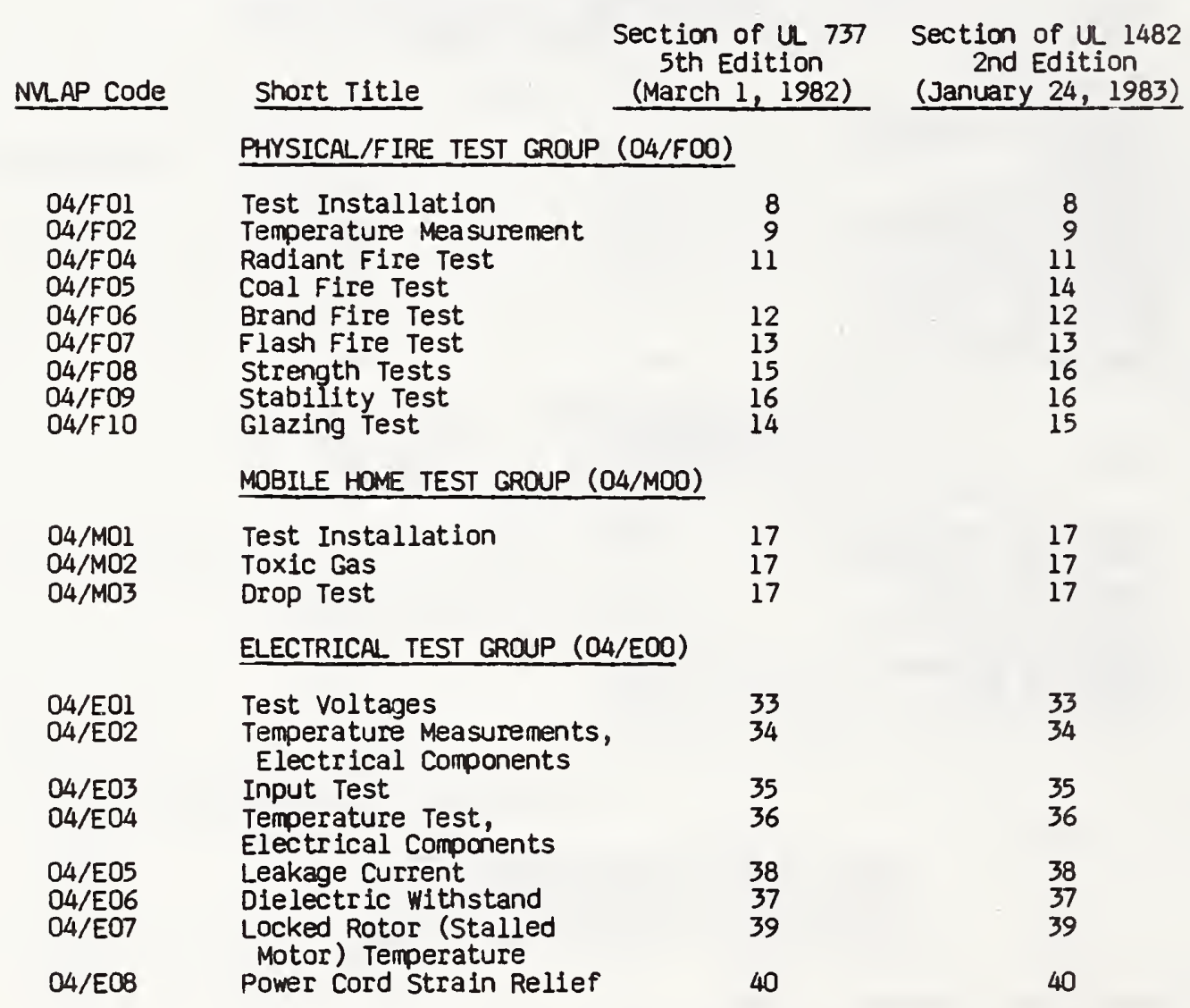


R. F. GEISSER \& ASSOCIATES, INC.

120 Pershing Street, P.O. Box 4526, East Providence, RI 02914 Bryon R. Holmes Phone: 401-438-7320

Accreditation Renewal Date: January 1, 1988

PHYSICAL/FIRE TEST GROUP

\begin{tabular}{|c|c|c|c|}
\hline LAP Code & Short Title & (November 9, 1982) & (January 24, 1983) \\
\hline $\begin{array}{l}04 / F 01 \\
04 / F 02 \\
04 / F 04 \\
04 / F 05 \\
04 / F 06 \\
04 / F 07 \\
04 / F 08 \\
04 / F 09 \\
04 / F 10\end{array}$ & $\begin{array}{l}\text { Test Installation } \\
\text { Temperature Measurement } \\
\text { Radiant Fire Test } \\
\text { Coal Fire Test } \\
\text { Brand Fire Test } \\
\text { Flash Fire Test } \\
\text { Strength Tests } \\
\text { Stability Test } \\
\text { Glazing Test }\end{array}$ & $\begin{array}{r}8 \\
9 \\
11 \\
12 \\
13 \\
15 \\
16 \\
14\end{array}$ & $\begin{array}{l}8 \\
9 \\
11 \\
14 \\
12 \\
13 \\
16 \\
16 \\
15\end{array}$ \\
\hline
\end{tabular}

Section of CSA Standard B 366.2-M1984

(ULC S627-M1984)

04/F11 Test Installation

$04 / F 12$

$04 / F 14$

$04 / F 16$

$04 / F 17$

$04 / F 18$

$04 / F 19$

04/F20

Temperature Mea surement

Radiant Fire Test

(April, 1984)

Brand Fire Test

Flash Fire Test

Strength Tests

Stability Test

Glazing Test

7.3

7.5

7.6

7.7

7.12

7.10

7.11

MOBILE HOME TEST GROUP

NULAP Code Short Title

Section of UL 737 Section of UL 1482

5th Edition 2nd Edition

$\begin{array}{ll}04 / \mathrm{MO1} & \text { Test Installation } \\ 04 / \mathrm{MO2} & \text { Toxic Gas } \\ 04 / \mathrm{MO3} & \text { Drop Test }\end{array}$

(November 9, 1982) (January 24, 1983)

$\begin{array}{ll}17 & 17 \\ 17 & 17 \\ 17 & 17\end{array}$

Section of CSA Standard B 366.2-M1984

(ULC S627-M1984)

(April, 1984)

04/MO4

04/MO5

04/MO6

Test Installation

Toxic Cas

12

Drop Test

12

\section{ELECTRICAL TEST GROUP}

\begin{tabular}{cl} 
NVLAP Code & Short Title \\
\hline 04/E01 & Test Voltages \\
04/E02 & $\begin{array}{l}\text { Temperature Measurements, } \\
\text { Electrical Components }\end{array}$ \\
04/E03 & Input Test \\
04/E04 & Temperature Test, \\
04/E05 & Electrical Components \\
04/E06 & Dielectric Withstand \\
04/E07 & Locked Rotor (Stalled \\
O4/E08 & Motor) Temperature \\
& Power Cord Strain Relief
\end{tabular}

Section of UL 737 Section of UL 1482

5th Edition 2nd Edition

(November 9, 1982) (January 24, 1983)

33

33

$34 \quad 34$

$35 \quad 35$

$36 \quad 36$

$38 \quad 38$

$37-37$

$39 \quad 39$

$40 \quad 40$ 


\begin{tabular}{|c|c|c|c|}
\hline & & $\begin{array}{c}\text { Section of CSA } \\
\text { C } 22.2 \text { No. } 3 \\
1979 \\
\end{array}$ & $\begin{array}{l}\text { Section of CSA } \\
\text { C 22.2 NO. } 113 \\
1982 \\
\end{array}$ \\
\hline O4/EO9 & $\begin{array}{l}\text { Temperature Measurements, } \\
\text { Electrical Components }\end{array}$ & 6.2 & 6.4 \\
\hline O4/E1O & $\begin{array}{l}\text { Temperature Test, } \\
\text { Electrical Components }\end{array}$ & 6.2 & 6.4 \\
\hline $\begin{array}{l}\text { O4/E11 } \\
\text { O4/E12 } \\
\text { O4/E13 }\end{array}$ & $\begin{array}{l}\text { Leakage Current } \\
\text { Dielectric Withstand } \\
\text { Power Cord Strain Relief }\end{array}$ & $\begin{array}{l}6.3 \\
6.4\end{array}$ & $\begin{array}{l}6.8 \\
6.5 \\
6.9\end{array}$ \\
\hline
\end{tabular}

NVLAP LAB CODE 0247

HDLLYTEX CARPET MILL, INC.

$505+$ N.E. Seventh Street, P.O. Box 369, Anadarko, OK 73005 Darlene McIntire Phone: 405-247-6641

Accreditation Renewal Date: April 1, 1987

\begin{tabular}{|c|c|}
\hline NVLAP Code & Designation \\
\hline $\begin{array}{l}03 / \mathrm{CO} 2 \\
03 / F 03\end{array}$ & $\begin{array}{l}\text { AATCC } 8 \\
16 \text { CFR Part } 1630\end{array}$ \\
\hline $\begin{array}{l}03 / \mathrm{SO} 1 \\
03 / \mathrm{SO} 2 \\
03 / \mathrm{SO3}\end{array}$ & $\begin{array}{l}\text { ASTM D1335 } \\
\text { ASTM D2646, sec. } 7 \\
\text { ASTM D3936, }\end{array}$ \\
\hline
\end{tabular}

Short Title

Colorfastness to Crocking

Surface Flamability

Test Procedure

Tuft Bind of Floor Coverings

Testing Backing Fabrics, Breaking Load

Delamination strength of Secondary Backing of

Pile floor coverings

NRLAP LAB COOE 0248

KNAUF FIBER GLASS RESEARCH LABORATORIES

240 Elizabeth Street, Shelbyville, IN 46176

Kerry VanArsdel Phone: 317-398-4434

Accreditation Renewal Date: April 1, 1987

\begin{tabular}{|c|c|}
\hline NVLAP Code & Designation \\
\hline $\begin{array}{l}01 / 002 \\
01 / 008 \\
01 / 009 \\
01 / 011\end{array}$ & $\begin{array}{l}\text { ASTM C167 } \\
\text { ASTM C } 302 \\
\text { ASTM C } 303 \\
\text { ASTM C } 356\end{array}$ \\
\hline $01 / 012$ & ASTM C411 \\
\hline $\begin{array}{l}01 / 013 \\
01 / \mathrm{SOI} \\
01 / \mathrm{TO1}\end{array}$ & $\begin{array}{l}\text { ASTM C519 } \\
\text { ASTM C165 } \\
\text { ASTM C177 }\end{array}$ \\
\hline $\begin{array}{l}\text { 01/T05 } \\
01 / T 06 \\
01 / T 09 \\
01 / T 10\end{array}$ & 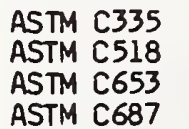 \\
\hline
\end{tabular}

Short Title

Thickness and density; Blanket and batt

Density; Preformed pipe insulation

Density; Preformed block insulation

Linear shrinkage; Soaking heat;

Preformed high temperature insulation

Hot-sur face performance;

High temperature insulation

Density; Loose-fill (fibrous)

Compressive properties; Thermal insulation (proc. A)

Thermal transmission properties;

Low-temperature guarded hot plate

Thermal conductivity; Pipe insulation

Thermal transmission properties; Heat flow meter

Thermal resistance (Rec. Practice); Blanket (mineral fiber)

Thermal resistance (Rec. Practice); Loose-fill (fibrous) 
WARNOCK HERSEY INTERNATIONAL, INC.

8612 Fairway Place, Middleton, WI 53562

F. D. Curkeet Phone: 608-836-4400

Accreditation Renewal Date: January 1, 1988

PHYSICAL/FIRE TEST GROUP

\begin{tabular}{|c|c|c|c|}
\hline NVLAP Code & Short Title & $\begin{array}{c}\text { 5th Edition } \\
\text { (November 9, 1982) }\end{array}$ & $\begin{array}{l}\text { 2nd Edition } \\
\text { (January 24, 1983) }\end{array}$ \\
\hline $\begin{array}{l}04 / \mathrm{FO} \\
04 / \mathrm{FO2} \\
04 / \mathrm{FO4} \\
04 / \mathrm{FO5} \\
04 / \mathrm{FO} 06 \\
04 / F 07 \\
04 / F 08 \\
04 / F 09 \\
04 / F 10\end{array}$ & $\begin{array}{l}\text { Test Installation } \\
\text { Temperature Measurement } \\
\text { Radiant Fire Test } \\
\text { Coal Fire Test } \\
\text { Brand Fire Test } \\
\text { Flash Fire Test } \\
\text { Strength Tests } \\
\text { Stability Test } \\
\text { Glazing Test }\end{array}$ & $\begin{array}{l}8 \\
9 \\
11 \\
12 \\
13 \\
15 \\
16 \\
14\end{array}$ & $\begin{array}{l}8 \\
9 \\
11 \\
14 \\
12 \\
13 \\
16 \\
16 \\
15\end{array}$ \\
\hline
\end{tabular}

Section of CSA Standard B 366.2 M1984

$\begin{array}{llc}\text { 04/F11 } & \text { Test Installation } & \text { (Apr11, 1984) } \\ \text { 04/F12 } & \text { Temperature Measurement } & 7.2 \\ \text { 04/F14 } & \text { Radiant Fire Test } & 7.5 \\ \text { 04/F16 } & \text { Brand Fire Test } & 7.6 \\ \text { 04/F17 } & \text { Flash Fire Test } & 7.7 \\ \text { 04/F18 } & \text { Strength Tests } & 7.12 \\ \text { 04/F19 } & \text { Stability Test } & 7.10 \\ \text { 04/F20 } & \text { Glazing Test } & 7.11\end{array}$

MOBILE HOME TEST GROUP

\begin{tabular}{|c|c|c|c|}
\hline NVLAP Code & Short Title & $\begin{array}{c}\text { 5th Edition } \\
\text { (November 9, 1982) }\end{array}$ & $\begin{array}{l}\text { 2nd Edit } \\
\text { (January 24, }\end{array}$ \\
\hline $\begin{array}{l}\text { 04/MO1 } \\
\text { 04/MO2 } \\
\text { 04/MO3 }\end{array}$ & $\begin{array}{l}\text { Test Installation } \\
\text { Toxic Gas } \\
\text { Drop Test }\end{array}$ & $\begin{array}{l}17 \\
17 \\
17\end{array}$ & $\begin{array}{l}17 \\
17 \\
17\end{array}$ \\
\hline
\end{tabular}

Section of CSA Standard $6366.2+M 1984$

(ULC s627-M1984)

(April, 1984)

04/MO4

04/MO5

Test Installation

12

Toxic Gas

12

04/M06

Drop Test

12

ELECTRICAL TEST GROUP

\begin{tabular}{|c|c|c|c|}
\hline NVLAP Code & Short Title & $\begin{array}{l}\text { dition } \\
9,1982)\end{array}$ & $\begin{array}{c}\text { 2nd Edition } \\
\text { (January 24, 1983) }\end{array}$ \\
\hline $\begin{array}{l}\text { O4/EO1 } \\
\text { 04/E02 }\end{array}$ & $\begin{array}{l}\text { Test Voltages } \\
\text { Temperature Measurements, } \\
\text { Electrical Components }\end{array}$ & $\begin{array}{l}33 \\
34\end{array}$ & $\begin{array}{l}33 \\
34\end{array}$ \\
\hline $\begin{array}{l}\text { O4/E03 } \\
\text { O4/E04 }\end{array}$ & $\begin{array}{l}\text { Input Test } \\
\text { Temperature Test, } \\
\text { Electrical Components }\end{array}$ & $\begin{array}{l}35 \\
36\end{array}$ & $\begin{array}{l}35 \\
36\end{array}$ \\
\hline $\begin{array}{l}\text { 04/E05 } \\
\text { O4/E06 } \\
\text { O4/E07 }\end{array}$ & $\begin{array}{l}\text { Leakage Current } \\
\text { Dielectric Withstand } \\
\text { Locked Rotor (Stalled } \\
\text { Motor) Temperature }\end{array}$ & $\begin{array}{l}38 \\
37 \\
39\end{array}$ & $\begin{array}{l}38 \\
37 \\
39\end{array}$ \\
\hline O4/E08 & Power Cord Strain Relief & 40 & 40 \\
\hline
\end{tabular}




\begin{tabular}{|c|c|c|c|}
\hline & & $\begin{array}{c}\text { Section of CSA } \\
\text { C } 22.2 \text { No. } 3 \\
1979 \\
\end{array}$ & $\begin{array}{r}\text { Section of CSA } \\
\text { C 22. } 2 \text { No. } 113 \\
1982 \\
\end{array}$ \\
\hline 04/E09 & $\begin{array}{l}\text { Temperature Measurements, } \\
\text { Electrical Components }\end{array}$ & 6.2 & 6.4 \\
\hline O4/E10 & $\begin{array}{l}\text { Temperature Test, } \\
\text { Electrical Components }\end{array}$ & 6.2 & 6.4 \\
\hline $\begin{array}{l}04 / E 11 \\
04 / E 12 \\
04 / E 13\end{array}$ & $\begin{array}{l}\text { Leakage Current } \\
\text { Dielectric Withstand } \\
\text { Power Cord Strain Relief }\end{array}$ & $\begin{array}{l}6.3 \\
6.4\end{array}$ & $\begin{array}{l}6.8 \\
6.5 \\
6.9\end{array}$ \\
\hline
\end{tabular}

NVLAP LAB CODE O250

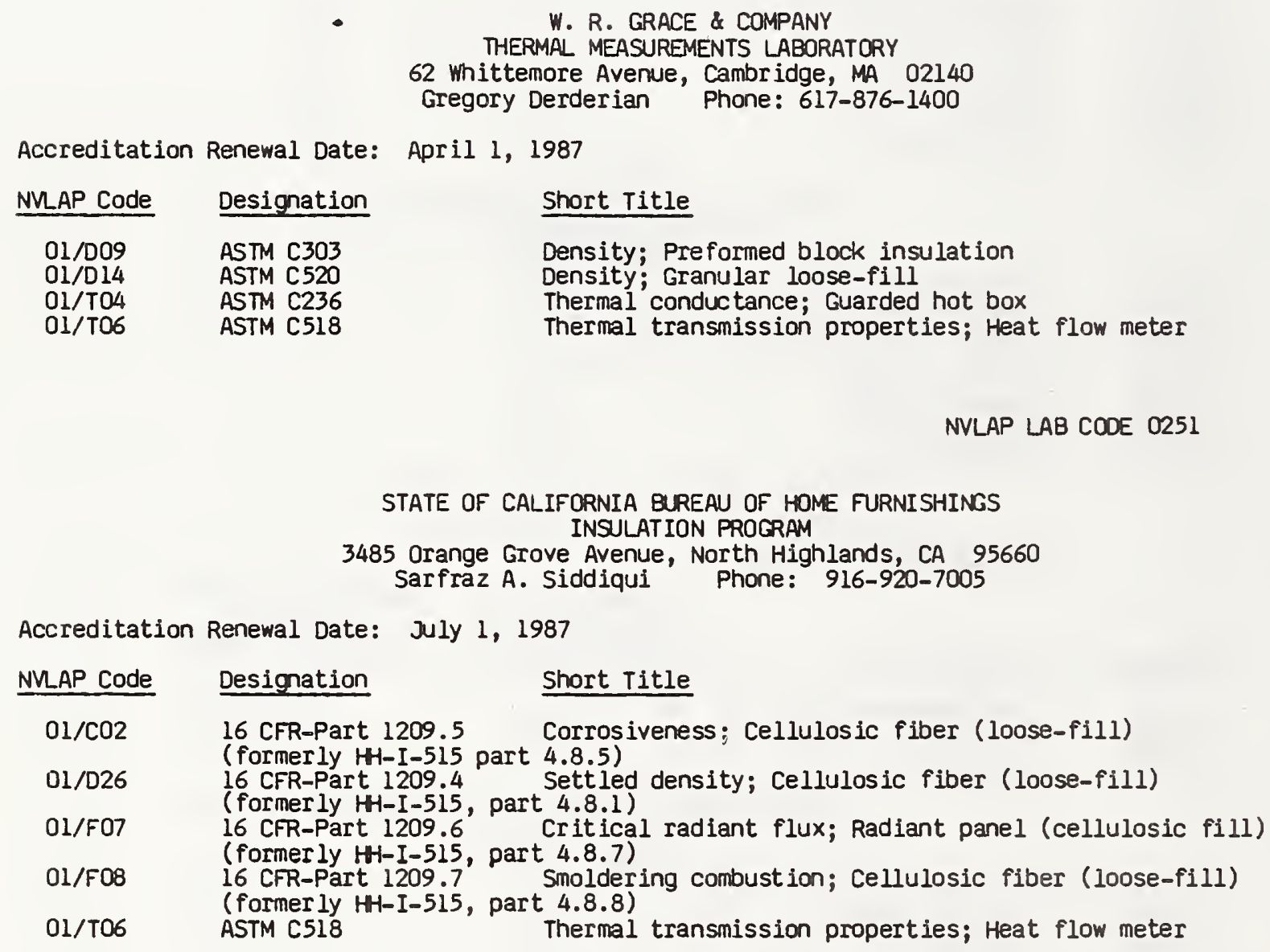

NVLAP LAB CODE 0252

D/L LABORATORIES

116 East 16th Street, New York, NY 10003

Saul Spindel Phone: 212-777-4410.

Accreditation Renewal Date: October 1, 1987

\begin{tabular}{cll} 
NLAP Code & & Designation \\
\cline { 1 - 1 } 09/A01 & & ASTM D56 \\
09/A02 & & ASTM D93 \\
09/A03 & & ASTM D153 \\
09/A04 & & ASTM D185 \\
$09 / A 05$ & & ASTM D281 \\
09/A07 & & ASTM D523
\end{tabular}

\section{Short Title}

Flash point by Tag Closed Tester

Flash Point by Pensky-Martens Closed Tester, Method A \& B Specific Gravity of Pigments

Coarse Particles in Pigments, Pastes and Paints

Oil Absorption of Pigments by Spatula Rub-out

Specular Gloss 
ASTM D1210 ASTM D1212

ASTM D1296

ASTM D1310 ASTM D1400

09/A21

O9/A22

09/A23

09/A24

09/A25

09/A26

09/A28

ASTM D 1475

ASTM D1544

ASTM D1729

ASTM D2244

ASTM D3278

ASTM D 3363

ASTM D3793

ASTM D4061

ASTM D4212

ASTM E97

09/BO1

09/BO2

09/B03

09/BO4

09/B05

09/B06

09/807

09/808

09/809

09/日10

09/B11

09/B12

$09 / 813$

09/B14

09/日15

09/B16

09/日17

$09 / 818$

09/日19

$09 / 920$

$09 / 1823$

$09 / 824$

09/825

09/826

09/日27

09/829

09/B30

09/B31

09/B32

09/日33

09/834

$09 / 835$

09/B37

09/838

09/B39

09/840

$09 / 841$

$09 / 842$

ASTM D821
ASTM E313

ASTM D279

ASTM D332

ASTM D344

ASTM D610

ASTM D659

ASTM D660

ASTM D661

ASTM D662

ASTM D711

ASTM D714

ASTM D772

ASTM D868

ASTM D869

ASTM D870

ASTM D913

ASTM D968

ASTM D969

ASTM D1308

ASTM D1 309

ASTM D1640

ASTM D1737

ASTM D2197

ASTM D2243

ASTM D2248

ASTM D2486

ASTM D2801

ASTM D2805

ASTM D3273

ASTM D3274

ASTM D3450 ASTM D3456 ASTM D4060

ASTM D4062 ASTM D4213

ASTM D4214

Fed. Std. 141

Method 4494

Fed. Std. 141

Method 4061
Consistency of Paints; Stormer Viscometer Procedure A \& B

Dry Film Thickness of Organic Coatings

Dry Film Thickness of Non-magnetic Coatings Applied to a Ferrous Base, Method A \& $B$

Viscosity of Paints, Varnishes, and Lacquers by Ford Viscosity Cup

Fineness of Dispersion of Pigment-Vehicle Systems Het Film Thickness of Organic Coatings, Method A

Odor of Volatile Solvents and Diluents

Flash-Point of Liquids by Tag open-Cup Apparatus

Dry Film Thickness of Non-conductive Coatings Applied to a Nonferrous Metal Base

Density of Paint, Varnish, Lacquer, and Related Products Color of Transparent Liquids (Gardner Color Scale)

Visual Evaluation of Color Differences of Opaque Materials

Instrumental Evaluation of Color Difference of Opaque Materials

Flash Point of Liquids: Setaflash Closed Tester Method A \& B

Film Hardness by Pencil Test

Low-Temperature Coalescence of Latex Paint Films

Specific Luminance of Horizontal Coatings

Viscosity by Dip-Type Viscosity Cups

45- deg, 0-deg Directional Reflectance Factor of Opaque Specimens by Broad-Band Filter Reflectometry

Indexes of Whiteness and Yellowness of Near-White Opaque Materials

Bleeding of Pigments, Method A

Tinting Strength of White Pigments, Method A

Re lative Dry Hiding Power of Paints

Rusting on Painted Steel Surfaces

Chalking of Exterior Paints

Checking of Exterior Paints

Cracking of Exterior Paints

Erosion of Exterior Paints

No-Pick-Up Time of Traffic Paint

Blistering of Paints

Flaking (Scaling) of Exterior Paints

Abrasion, Erosion or a Combination of Both in Road

Service Tests of Traffic Paints

Bleeding of Traffic Paint

Settling of Traffic Paint

Water Immersion Test of Organic Coatings on Steel

Chipping of Traffic Paint

Abrasion Resistance of Organic coatings by the

Falling Abrasive Tester, Method A \& B

Bleeding of Traffic Paint

Effect of Household Chemicals on Clear and

Settling Properties of Traffic Paint During

Drying, Curing, or Film formation of Organic

Elongation of Attached Organic Coatings with

Cylindrical Mandrel Apparatus

Adhesion of Organic Coatings, Method A

Freeze-Thaw Resistance of Latex and Emulsion Paints

Detergent Resistance of Organic Finishes

Scrub Resistance of Interior Latex Flat Wall Paints

Leveling Characteristics of Paints by Draw-Down Method

Hiding Power of Paints

Resistance to Growth of Mold on the Sur face of

Interior Coatings in an Environmental Chamber

Surface Disfigurement of Paint Films by Fungal

Growth or Soil and Dirt Accumulation

Washability Properties of Interior Architectural Coatings

Susceptability of Paint Films to Microbioligical Attack

Abrasion Resistance of Organic Coatings by the

Taber Abraser

Leveling of Paints by Draw-Down Method

Wet Abrasion Resistance of Interior Paint by Weight Loss Chalking of Exterior Paint Films, Method $A, B, C, D$ \& $E$

Sag Test (Multinotch Blade)

Drying Time 
09/C02

ASTM D95

09/C06

$09 / C 07$

$09 / \mathrm{COB}$

09/C09

09/Cll

$09 / \mathrm{Cl2}$

$09 / \mathrm{C}_{22}$

$09 / C 26$

$09 / C 27$

$09 / C 28$

$09 / C 29$

$09 / C 30$

$09 / C 37$

$09 / C 39$

$09 / C 40$

$09 / 001$

09/002

09/003

09/004

09/005

$09 / 006$

09/007

09/010

$09 / 011$

$09 / 013$

09/014

09/016

13/001

$13 / 002$

$13 / 003$

$13 / 004$

$13 / 005$

$13 / 006$

$13 / 007$

$13 / 008$

13/009

$13 / 010$

$13 / 011$

$13 / 012$

$13 / 013$

$13 / 014$

$13 / 015$

$13 / 016$

$13 / 017$

$13 / 018$

$13 / 019$

$13 / 020$

$13 / 021$

$13 / 022$

$13 / 023$

$13 / 024$

$13 / 025$

$13 / 026$

$13 / 027$

$13 / 028$

$13 / 029$

$13 / 030$
ASTM D1078

ASTM DI133

ASTM D1208

ASTM D1259

ASTM D1353

ASTM D1364

ASTM D1644

ASTM D2369

ASTM D2371

ASTM D2697

ASTM 02698

ASTM D2832

.1STM D3723

ASTM D3960

ASTM 04017

ASTM B117

ASTM 0609

ASTM D822

ASTM D823

ASTM D1006

ASTM D1014

ASTM D1654

ASTM D2247

ASTM D2372

ASTM 03924

ASTM G23

ASTM G53

ASTM C-510

ASTM C-603

ASTM C-639

ASTM C-661

ASTM C-679

ASTM C-681

ASTM C-711

ASTM C-712

ASTM C-713

ASTM C-718

ASTM C-719

ASTM C-731

ASTM C-732

ASTM C-733

ASTM C-734

ASTM C-736

ASTM C-741

ASTM C-742

ASTM C-792

ASTM C-793

ASTM C-794

ASTM C-910

ASTM D-2202

ASTM D-2203

ASTM D-2376

ASTM D-2377

ASTM D-2450

ASTM D-2451

ASTM D-2452

ASTM D-2453
Water in Petroleum Products and Bitumirious

Materials by Distillation

Distillation Range of Volatile Organic Liquids

Kauri-Butanol Value of Hydro-carbon Solvents

Common Properties of Certain Pigments

Nonvolatile Content of Resin Solutions, Method A \& B

Nonvolatile Matter in Volatile Solvents for Use in

Paint, Varnish, Lacquer and Related Products

Water in Volatile Solvents (Fischer Reagent Titration Method)

Nonvolatile Content of Vamishes, Methods $A$ \& $B$

Volatile content of Paints, Procedure A \& B

Pigment content of Solvent-Type Paints

Volume Nonvolatile Matter in Clear or Pigmented Coatings

Pigment Content of Solvent-Type Paints by

High-Speed Centrifuging

Nonvolatile Content of Paint and Paint Materials

Pigment Content of Water-Emulsion Paints by

Low-Temperature Ashing

Volatile Organic contents (VOC) Paints and Related Coatings Water in Paints and Paint Materials by Karl Fischer Method

Salt Spray (Fog) Testing

Preparation of Steel Panels for Testing Paints

Varnish, Lacquer, and Related Products, Method A, B, C, \& D Operating Light-and-Water-Exposure Apparatus

(Carbon-Arc Type) for Testing Paint, Vamish, Lacquer, and Related Products

Producing Films of Uniform Thickness of Paint

Vamish, Lacquer, and Related Products on

Test Panels, Method B \& D

Exterior Exposure Tests of Paints on Wood

Exterior Exposure Tests of Paints on Steel, Method B,C,D \& E

Painted or coated Specimens Subjected to Corrosive

Environments, Procedures $A$ \& $B$

Coated Metal Specimens at $100 \%$ Relative Humidity

Separation of Vehicle Solvent-Type Paints

standard Environment for Conditioning and Testing

Paint, Vamish, Lacquer, and Related Materials

Operating Light-Exposure Apparatus (Carbon-Arc Type) with

and Without Water for Exposure of Nonmetallic Materials, Method 1, 2, 3, \& 4

Operating Light and water-Exposure Apparatus (Fluorescent UV

Condensation Type) for Exposure of Nonmetallic Materials

staining and color change

Extrusion Rate and Application Life

Rheological (Flow) Properties

Indentation Hardness by Durometer

Tack-Free Time

Volatility

Low-Temperature Flexibility and Tenacity

Bubbling

Slump

UV-Cold Box Exposure

Adhesion and Cohesion Under Cyclic Movement

Extrudibility, After Package Aging

Aging Effects of Artificial Weathering

Volume Shrinkage

Low-Temperature Flexibility After Artificial Weathering

Extension-Recovery and Adhesion After Artificial Weathering

Accelerated Aging

Degree of set

Effects of Heat Aging on Weight Loss, Cracking, and Chalking

Effects of Accelerated Weathering

Adhesion-in-Peel

Bond and cohesion

Slump

Staining

Slump

Tack-Free Time

Bond

Degree of Set

Extrudibility

Shrinkage and Tenacity 


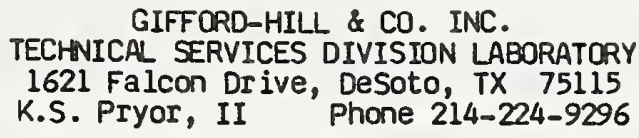

Accreditation Renewal Date: January 1, 1988

\begin{tabular}{|c|c|}
\hline $\begin{array}{l}\text { NVLAP Test } \\
\text { Method Cade }\end{array}$ & Test Metho \\
\hline $02 / \mathrm{GO}$ & $\begin{array}{l}\text { ASTM C C } 11 \\
\text { ASTM C172 } \\
\text { ASTM C143 } \\
\text { ASTM C138 } \\
\text { ASTM C231 } \\
\text { ASTM C173 }\end{array}$ \\
\hline $\begin{array}{l}\text { O2/AO1 } \\
\text { O2/A02 }\end{array}$ & $\begin{array}{l}\text { ASTM C39 } \\
\text { ASTM C617 }\end{array}$ \\
\hline
\end{tabular}

Making and curing Concrete Test Specimens in the field Sampling Freshly Mixed Concrete Slump of Portland Cement Concrete Unit Weight, Yield, and Air Content (Gravimetric) Air Content of Freshly Mixed Concrete by the Pressure Method Air Content Volumetric Method Compressive Strength of Cylindrical Concrete Specimens Capping Cylindrical Specimens

NVLAP LAB COOE 0255

\author{
UNDERWRITERS LABORATORIES INC. \\ 333 Pfingsten Road, Northbrook, IL 60062 \\ Steve Mazzoni Phone: 516-271-6200
}

Accreditation Renewal Date: October 1, 1987

NVAP Test

Method Code

Test Method Designation

12/CO1 Conducted Emissions, Power Lines, $450 \mathrm{KHz}$ to $30 \mathrm{MHz}$

FCC Method - 47 CFR Part 15 Subpart J

12/RO1 Radiated Emissions, $30 \mathrm{MHz}$ to $1000 \mathrm{MHz}$

FCC Method - 47 CFR Part 15 Subpart J

NVLAP LAB COOE 0256

WESTERN ELECTRO-ACOUSTIC LABORATORY, INC.

1711 16th Street, Santa Monica, CA 90404

Jose C. Ortega Phone: 213-870-9268

Accreditation Renewal Date: April 1, 1987

NMAP Code Designation

08/P03 ASTM C423

08/P06 ASTM E90

\section{Short Title}

Sound Absorption and Sound Absorption Coefficients Airborne Sound Transmission Loss of Building Partitions

NVLAP LAB CODE 0257

GAI CONSULTANTS, INC.

570 Beatty Road, Monroeville, PA 15146

Charles T. Ford Phone: 412-856-6400

Accreditation Renewal Date: July 1, 1987

\begin{tabular}{|c|c|}
\hline NVAP Code & Designation \\
\hline $02 / \mathrm{GO}$ & $\begin{array}{l}\text { ASTM C C 1 } \\
\text { ASTM C172 } \\
\text { ASTM C143 } \\
\text { ASTM C138 } \\
\text { ASTM C } 231 \\
\text { ASTM C } 173\end{array}$ \\
\hline O2/AO1 & ASTM C 39 \\
\hline
\end{tabular}

Short Title

Making and Curing Test Specimens

Sampling Freshly Mixed Concrete

Slump of Port land Cement Concrete

Unit Weight, Yield, and Air Content

Air Content-Pressure Method

Content-Volumetric Method

Compressive Strength of Cylindrical Specimens 
THE CELOTEX CORPORATION, TRACY PLANT

400 West Gandy Dancer Drive, P.O. Box 1500, Tracy, CA 95376 Robert E. Herrell Phone: 209-836-4440

Accreditation Renewal Date: July 1, 1987

NVAP Code Designation

$01 /$ TO6 ASTM C518

\section{Short Title}

Thermal transmission properties; Heat flow meter

MAOMILLAN BLOEDEL INC.

TECHNICAL DEPARTMENT TESTING LABORATORIES
P.O. Box 336, PIne Hil1, AL 36769
G. S. Overstreet Phone: 205-963-4391

Accreditation Renewal Date: July 1, 1987

NVLAP Test Method

Code Designation

\section{Short Title}

\section{Paper and Paperboard}

\begin{tabular}{|c|c|}
\hline O9/E02 & TAPPI T402-OM \\
\hline O9/E03 & $\begin{array}{l}\text { TAPPI T403-0S } \\
\text { ASTM } 0774\end{array}$ \\
\hline $\begin{array}{l}\text { 09/E05 } \\
\text { 09/E06 } \\
\text { O9/E07 }\end{array}$ & $\begin{array}{l}\text { TAPPI T410-OM } \\
\text { TAPPI T411-OM } \\
\text { TAPPI T412-OM } \\
\text { ASTM } 0644\end{array}$ \\
\hline O9/E08 & $\begin{array}{l}\text { TAPPI T414-OM } \\
\text { ASTM } 0689\end{array}$ \\
\hline 09/E10 & TAPPI T435-OM \\
\hline 09/E12 & $\begin{array}{l}\text { TAPPI T459-OM } \\
\text { ASTM D2482 }\end{array}$ \\
\hline $09 / E 13$ & $\begin{array}{l}\text { TAPPI T460-OM } \\
\text { ASTM } 0726\end{array}$ \\
\hline 09/E17 & TAPPI T494-OM \\
\hline O9/E19 & TAPPI T538-PM \\
\hline $\begin{array}{l}\text { O9/E20 } \\
\text { O9/E21 }\end{array}$ & $\begin{array}{c}\text { TAPPI T809-OM } \\
\text { TAPPI T818-OM } \\
\text { ASTM D1164 }\end{array}$ \\
\hline
\end{tabular}

\section{Packaging}

$\begin{array}{ll}\text { 09/HOI } & \text { ASTM 0642 } \\ 09 / \mathrm{H} 23 & \text { TAPPI T6880M } \\ \text { 09/H24 } & \text { TAPPI T8020S } \\ 09 / \mathrm{H} 25 & \text { TAPPI T8030M } \\ 09 / \mathrm{H} 26 & \text { TAPPI Use fuI } \\ & \text { Method 807 } \\ 09 / \mathrm{H} 27 & \text { TAPPI T8080S } \\ 09 / \mathrm{H} 28 & \text { TAPPI T8100M } \\ 09 / \mathrm{H} 29 & \text { TAPPI T8110S } \\ 09 / \mathrm{H} 30 & \text { TAPPI T821PM }\end{array}$

Compression Test for Shipping Containers

Total Wax Content of Corrugated Paperboard

Orop Test for Fiberboard Shipping Containers

Puncture and Stiffness Test of Container Board Wet Shear Adhesion Test of Corrugated

Fiberboard (MBR)

Flat Crush Test of Corrugated Board

Bursting Strength of Corrugated and Solid Fiberboard

Edgewise Compressive Strength of Corrugated

Fiberboard (Short column Test)

Pin Adhesion of Corrguated Board by Selective Separation

Standard conditioning and ASTM D685 Testing Atmospheres

Paper, Board, Pulp Handsheets and Related Products

Gramnage of Paper and Paper-board (Weight per Unit Area)

Thickness (Caliper) of Paper and Paperboard

Internal Tearing Resistance of Paper

Hydrogen Ion Concentration (pH) of Paper Extracts(Hot Extraction Method)

Sur face Strength of Paper (Wax Pick Test)

Air Resistance of Paper

Tensile Breaking Properties of Paper and

(Using Constant Rate of Elongation Apparatus)

(air Flow Method)

Flat Crush of Corrugating Medium (CMT Test)

Ring Crush of Paperboard 
BASF STYROPOR TECHNICAL CENTER

Cranbury and South River Road, Jamesburg, NJ 08831

Mark C. Braemer Phone: 201-521-1600

Accreditation Renewal Date: October 1, 1987

\begin{tabular}{|c|c|}
\hline IVAP Code & Designation \\
\hline $\begin{array}{l}01 / \mathrm{SO} 2 \\
01 / \mathrm{Sll}\end{array}$ & $\begin{array}{l}\text { ASTM C203 } \\
\text { ASTM D1621 }\end{array}$ \\
\hline $01 / T 06$ & ASTM C518 \\
\hline
\end{tabular}

Short Title

Breaking load/flexural strength; Preformed block insulation Compressive properties; Rigid cellular plastics (proc. A-Crosshead)

Thermal transmission properties; Heat flow meter

\title{
NVLAP LAB CODE 0261
}

\author{
RADCO (RESOURCES APPLICATIONS, \\ DESIGNS \& CONTPOLS, INC.) \\ 16415 South Avalon Blvd., Gardena, CA 90248 \\ J. D. Waldman Phone: 213-532-3842
}

Accreditation Renewal Date: January 1, 1988

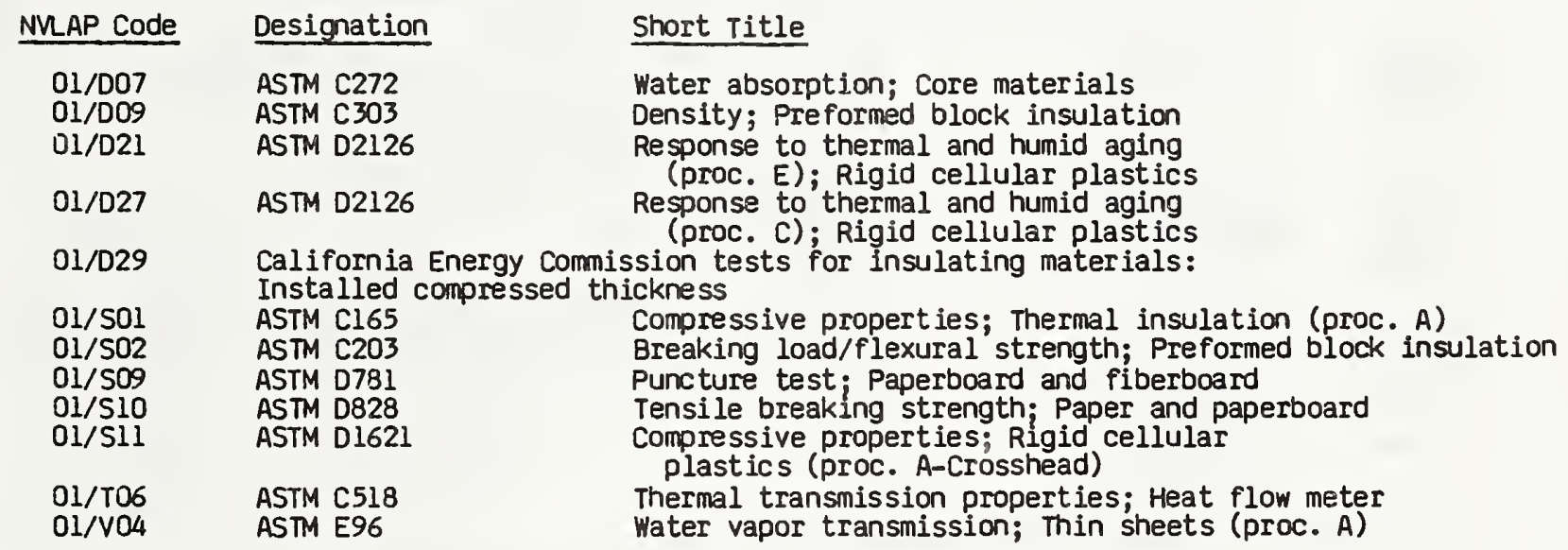

NVLAP LAB CODE 0263

WHITTAKER ANALYTICAL SERVICES

1231 South Lincoln Street, P.O. Box 825, Colton, CA 92324

Edward J. Holzrichter Phone: 714-825-6292

Accreditation Renewal Date: January 1, 1988

NMAP Test Method

Code Designation

Short Title

Paints and Related coatings

$\begin{array}{ll}\text { 09/A04 } & \text { ASTM D185 } \\ \text { 09/A05 } & \text { ASTM D281 } \\ \text { 09/AO7 } & \text { ASTM D523 } \\ \text { 09/A09 } & \text { ASTM D1005 } \\ \text { 09/A11 } & \text { ASTM D1200 } \\ \text { 09/A12 } & \text { ASTM D 1210 } \\ \text { 09/A16 } & \text { ASTM D1400 } \\ \text { 09/A17 } & \text { ASTM D1475 } \\ \text { 09/A18 } & \text { ASTM D1544 } \\ \text { 09/A19 } & \text { ASTM D1729 }\end{array}$

Coarse Particles in Pigments, Pastes and Paints

Oil Absorption of Pigments by Spatula Rub-out

Specular Gloss

Dry Film Thickness of Organic Coatings

Viscosity of Paints, Varnishes, and Lacquers by Ford Viscosity cup

Fineness of Dispersion of Pigment-Vehicle Systems

Dry Film Thickness of Non-conductive Coatings Applied to a Nonferrous Metal Base

Density of Paint, Varnish, Lacquer, and Related Products Color of Transparent Liquids (Gardner Color Scale)

Visual Evaluation of Color Differences of Opaque Materials 
Instrumental Evaluation of Color Difference of Opaque Materials

Flash Point of Liquids by Setaflash Closed Tester, Methods $A$ \& $B$

Film Hardness by Pencil Test

Viscosity by Dip-Type Viscosity Cups

45- deg, O-deg Directional Reflectance Factor of Opaque Specimens by Broad-Band Filter Reflectometry

Indexes of Whiteness and Yellowness of Near-White Opaque Materials

\section{Measurements of Performance and Performance Change}

$\begin{array}{ll}\text { 09/B05 } & \text { ASTM D659 } \\ 09 / B 06 & \text { ASTM D660 } \\ 09 / B 07 & \text { ASTM D661 } \\ 09 / B 08 & \text { ASTM D662 } \\ 09 / B 10 & \text { ASTM D714. } \\ 09 / B 11 & \text { ASTM D772 } \\ 09 / B 12 & \text { ASTM D821 } \\ 09 / B 14 & \text { ASTM D869 } \\ 09 / B 15 & \text { ASTM D870 } \\ 09 / B 16 & \text { ASTM D913 } \\ 09 / B 17 & \text { ASTM D968 } \\ 09 / B 18 & \text { ASTM D969 } \\ 09 / B 20 & \text { ASTM D1309 } \\ 09 / B 23 & \text { ASTM D1640 } \\ 09 / B 24 & \text { ASTM D1737 } \\ 09 / B 25 & \text { ASTM D2197 } \\ 09 / B 27 & \text { ASTM D2248 } \\ 09 / B 31 & \text { ASTM D2805 } \\ 09 / B 33 & \text { ASTM D3274 } \\ 09 / B 37 & \text { ASTM D4060 } \\ 09 / B 40 & \text { ASTM D4214 } \\ 09 / B 41 & \text { Fed. Std. } 141 \\ 09 / B 42 & \text { Method } 4494 \\ & \text { Fed. Std. } 141 \\ & \text { Method 4061 }\end{array}$

Chalking of Exterior Paints

Checking of Exterior Paints

Cracking of Exterior Paints

Erosion of Exterior Paints

Blistering of Paints

Flaking (Scaling) of Exterior Paints

Abrasion, Erosion or a Combination of Both

in Road Service Tests of Traffic Paints

Settling of Traffic Paint

Water Immersion Test of Organic coatings on Steel

Chipping of Traffic Paint

Abrasion Resistance of Organic Coatings by the

Falling Abrasive Tester, Methods A \& B

Bleeding of Traffic Paint

Settling Properties of Traffic Paint During

Drying, curing, or Film Formation of organic

Elongation of Attached Organic coatings with

Cylindrical Mandrel Apparatus

Adhesion of Organic Coatings, Method $B$

Detergent Resistance of Organic Finishes

Hiding Power of Paints

Surface Disfigurement of Paint Films by Fungal

Growth or Soil and Dirt Accumulation

Abrasion Resistance of Organic Coatings by Taber Abraser

Chalking of Exterior Paint Films, Methods $A, B, C, \& D$

Sag Test (Multinotch Blade)

Drying Time

Measurement of Chemical Properties and Compositions

$\begin{array}{ll}09 / C 02 & \text { ASTM D95 } \\ 09 / C 04 & \text { ASTM D563 } \\ 09 / C 06 & \text { ASTM D1078 } \\ 09 / C 07 & \text { ASTM D1133 } \\ 09 / C 09 & \text { ASTM D1259 } \\ 09 / C 10 & \text { ASTM D } 1306 \\ 09 / C 11 & \text { ASTM D } 1353 \\ 09 / C 14 & \text { ASTM D } 1397 \\ 09 / C 15 & \text { ASTM D1398 } \\ 09 / C 17 & \text { ASTM D1467 } \\ 09 / C 20 & \text { ASTM D1613 } \\ 09 / C 21 & \text { ASTM D } 1639 \\ 09 / C 22 & \text { ASTM D1644 } \\ 09 / C 23 & \text { ASTM D1652 } \\ 09 / C 26 & \text { ASTM D2369 } \\ 09 / C 27 & \text { ASTM D2371 } \\ 09 / C 29 & \text { ASTM D2698 } \\ 09 / C 30 & \text { ASTM D2832 } \\ 09 / C 31 & \text { ASTM D } 3009 \\ 09 / C 32 & \text { ASTM D } 3271\end{array}$

Water in Petroleum Products and Bituminous Materials by Distillation

Phthalic Anhydride Content of Alkyd Resins and Resin Solutions

Distillation Range of Volatile Organic Liquids

Kauri-Butanol Value of Hydro-carbon Solvents

Nonvolatile Content of Resin Solutions, Methods $A$ \& $B$

Phthalic Anhydride Content of Alkyd Resins and

Esters Containing Other Dibasic Acids (Gravimetric)

Nonvolatile Matter in Volatile Solvents for Use in

Paint, Varnish, Lacquer and Related Products

Unsaponifiable Matter in Alkyd Resins and Resins Solutions

Fatty Acid Content of Alkyd Resins and Alkyd Resin Solutions, Methods $A \& B$

Fatty Acids Used in Protective Coatings

Acidity in Volatile Solvents and Chemical Intermediates Used in Paint, Varnish, Lacquer and Related Products

Acid value of Organic coating Materials

Nonvolatile Content of Varnishes, Methods $A \& B$

Epoxy Content of Epoxy Resins

volatile content of Paints, Procedures $A$ \& $B$

Pigment Content of Solvent-Type Paints

Pigment Content of Solvent-Type Paints by

High-Speed Centrifuging

Nonvolatile Content of Paint and Paint Materials

composition of Turpentine by Gas Chromatography

Direct Injection of Solvent-Base Paints into a Gas

Chromatograph for Solvent Analysis 


$\begin{array}{ll}09 / C 34 & \text { ASTM D3335 } \\ 09 / C 35 & \text { ASTM D3624 } \\ 09 / C 36 & \text { ASTM D3718 } \\ 09 / C 39 & \text { ASTM D3960 }\end{array}$

Low Concentrations of Lead, Cadmium, and Cobalt in Paint by Atomic Absorption Spectroscopy

Low Concentrations of Mercury in Paint by Atomic Absorption Spectroscopy

Low Concentrations of Chromium in Paint by Atomic Absorption Spectroscopy

volatile Organic contents ( $V O C)$ of Paints and Related Coatings

\section{Test Sample Conditioning and Preparation}

$\begin{array}{ll}\text { 09/D01 } & \text { ASTM B117 } \\ \text { 09/D07 } & \text { ASTM D1654 } \\ & \\ 09 / D 10 & \text { ASTM D2247 } \\ 09 / D 11 & \text { ASTM D2372 } \\ 09 / 016 & \text { ASTM G53 }\end{array}$

Salt Spray (Fog) Testing

Painted or Coated Specimens Subjected to Corrosive Environments, Procedures A \& B

Coated Metal Specimens at 100\% Relative Humidity

Separation of vehicle Solvent-Type Paints

operating Light- and Water-Exposure Apparatus

(Fluorescent UV-Condensation Type) for

Exposure of Nonmetallic Materials

NLAP LAB CODE 0264

SHELTON RESEARCH, INC.

1517 Pacheco Street, P.O. Box 5235, Santa Fe, 87502

Richard S. Blackbum Phone: 505-983-9457

Accreditation Renewal Date: January 1, 1988

PHYSICAL/FIRE TEST GROUP

\begin{tabular}{|c|c|}
\hline NVLAP Code & Short Title \\
\hline $\begin{array}{l}\text { 04/FO1 } \\
04 / F 02 \\
\text { 04/F04 } \\
04 / F 05 \\
\text { O4/F06 } \\
\text { 04/F07 } \\
\text { 04/F08 } \\
04 / F 09 \\
\text { 04/F10 }\end{array}$ & $\begin{array}{l}\text { Test Installation } \\
\text { Temperature Measurement } \\
\text { Radiant Fire Test } \\
\text { Coal Fire Test } \\
\text { Brand Fire Test } \\
\text { Flash Fire Test } \\
\text { Strength Tests } \\
\text { Stability Test } \\
\text { Glazing Test }\end{array}$ \\
\hline
\end{tabular}

Section of UL 737 Section of UL 1482

5 th Edition 2nd Edition

(November 9, 1982) (January 24, 1983)

ELECTRICAL TEST GROUP

$\begin{array}{rr}8 & 8 \\ 9 & 9 \\ 11 & 11 \\ 12 & 14 \\ 13 & 12 \\ 15 & 13 \\ 16 & 16 \\ 14 & 16 \\ & 15\end{array}$

\begin{tabular}{|c|c|c|c|}
\hline NMLAP Code & Short Title & $\begin{array}{c}\text { Section of UL } 737 \\
\text { 5th Edition } \\
\text { (November 9, 1982) } \\
\end{array}$ & $\begin{array}{c}\text { Section of UL } 1482 \\
\text { 2nd Edition } \\
\text { (January 24, 1983) } \\
\end{array}$ \\
\hline $\begin{array}{l}\text { O4/EO1 } \\
\text { O4/EO2 }\end{array}$ & $\begin{array}{l}\text { Test Voltages } \\
\text { Temperature Measurements, } \\
\text { Electrical Components }\end{array}$ & $\begin{array}{l}33 \\
34\end{array}$ & $\begin{array}{l}33 \\
34\end{array}$ \\
\hline $\begin{array}{l}\text { O4/E03 } \\
\text { O4/EO4 }\end{array}$ & $\begin{array}{l}\text { Input Test } \\
\text { Temperature Test, } \\
\text { Electrical Components }\end{array}$ & $\begin{array}{l}35 \\
36\end{array}$ & $\begin{array}{l}35 \\
36\end{array}$ \\
\hline $\begin{array}{l}\text { O4/E05 } \\
\text { O4/E06 } \\
\text { O4/EO7 }\end{array}$ & $\begin{array}{l}\text { Leakage Current } \\
\text { Dielectric Withstand } \\
\text { Locked Rotor (Stalled } \\
\text { Motor) Temperature }\end{array}$ & $\begin{array}{l}38 \\
37 \\
39\end{array}$ & $\begin{array}{l}38 \\
37 \\
39\end{array}$ \\
\hline O4/E08 & Power Cord Strain Relief & 40 & 40 \\
\hline
\end{tabular}


WEYERHAEUSER TECHNOLOGY CENTER

Mail Stop WTC 1B14, Tacoma, WA 98477

Tom Friberg Phone: 206-924-6204

Accreditation Renewal Date: April 1, 1987

$\begin{array}{ll}\text { NVAP } & \text { Test Method } \\ \text { Code } & \text { Designation }\end{array}$

Paper and Paperboard

\begin{tabular}{|c|c|}
\hline O9/E02 & TAPPI T402-OM \\
\hline O9/E03 & $\begin{array}{l}\text { TAPPI T403-OS } \\
\text { ASTM } 0774\end{array}$ \\
\hline O9/E05 & TAPPI T410-OM \\
\hline 09/E06 & TAPPI T411-O \\
\hline 09/E07 & $\begin{array}{l}\text { TAPPI T412-0 } \\
\text { ASTM } 0644\end{array}$ \\
\hline O9/E08 & TAPPI T414-OM \\
\hline O9/E09 & TAPPI T425-OM \\
\hline 09/EIl & TAPPI T452-OM \\
\hline O9/E12 & $\begin{array}{l}\text { TAPPI T459-OM } \\
\text { ASTM } 02482\end{array}$ \\
\hline 09/El3 & $\begin{array}{l}\text { TAPPI T460-OM } \\
\text { ASTM } 0726\end{array}$ \\
\hline 09/E15 & TAPPI T480-OS \\
\hline 09/E16 & TAPPI T489-OS \\
\hline & \\
\hline 09/E 18 & TAPPI T511-OM \\
\hline 09/E19 & TAPPI T538-PM \\
\hline O9/E20 & TAPPI T809-OM \\
\hline 09/E21 & $\begin{array}{l}\text { TAPPI T818-0 } \\
\text { ASTM D1164 }\end{array}$ \\
\hline
\end{tabular}

Pressure Sensitive Tapes

$\begin{array}{ll}09 / G 01 & \text { ASTM D } 3330, \\ & \text { D } 3330 M \\ 09 / G 02 & \text { ASTM D } 3652 \\ 09 / G 03 & \text { ASTM D } 3654, \\ & \text { D } 3654 M \\ 09 / G 04 & \text { ASTM D } 3662 \\ 09 / G 05 & \text { ASTM D } 3715\end{array}$

Packaging

$\begin{array}{ll}09 / \mathrm{HO1} & \text { ASTM } 0642 \\ 09 / \mathrm{HO2} & \text { ASTM D895 } \\ 09 / \mathrm{HO3} & \text { ASTM D1008 }\end{array}$

\section{Short Title}

Paper and Related Products

Standard conditioning and ASTM D685 Testing Atmospheres for Paper, Board, Pulp Handsheets and Related Products Bursting Strength of Paper

Grammage of Paper and Paper-board (Weight per Unit Area) Thickness (Caliper) of Paper and Paperboard

Moisture in Paper and Paperboard

Internal Tearing Resistance of Paper

Opacity of Paper (15/Diffuse Illuminant A, 89\% Reflectance Backing and Paper Backing)

Brightness of Pulp, Paper and Paperboard (Directional Reflectance at $457 \mathrm{~nm}$ )

Sur face Strength of Paper (Wax Plck Test)

Alr Resistance of Paper

Specular Gloss of Paper and Paper-board at 75 Degrees Stiffness of Paperboard

Tensile Breaking Propertles of Paper and Paperboard (Using Constant Rate of Elongation Apparatus)

Folding Endurance of Paper (MIT) Tester

Sheffield Smoothness of Paper and Paperboard (air Flow Method)

Flat Crush of Corrugating Medium (CMT Test)

Ring Crush of Paperboard

Test for Peel Adhesion of Pressure-Sensitive Tape at 180-deg Angle

Test for Thickness of Pressure-Sensitive and Gummed Tapes Test for Holding Power of Pressure-Sensitive Tapes

Test for Bursting Strength of Pressure-Sensitive Tapes

Practice for Quality Assurance of Pressure-Sensitive Tapes

Compression Test for Shipping Containers

Test for Water Vapor Permeability of Packages

Tests for Water Vapor Transmission of Shipping Containers

Federal Test Method Standard 101C for Preservation, Packaging, and Packaging Materials:

$\begin{array}{ll}\text { 09/HO7 } & \text { Method } 5005.1 \\ 09 / \mathrm{HOB} & \text { Method } 5007.1 \\ 09 / \mathrm{HOS} & \text { Method } 5008.1 \\ \text { 09/H19 } & \text { Method 5019.1 } \\ \text { 09/H20 } & \text { Method 5020.1 } \\ \text { 09/H27 } & \text { TAPPI T8080S } \\ \text { 09/H28 } & \text { TAPPI T8100M } \\ \text { 09/H29 } & \text { TAPPI T8110S } \\ 09 / \mathrm{H} 30 & \text { TAPPI T821PM }\end{array}$

Cornerwise Drop (Rotational) Test

Drop Test (Free Fall)

Edgewise Drop (Rotational) Test

vibration (Repetitive Shock) Test

Vibration (Sinusoidal Motion) Test

Flat Crush Test of Corrugated Board

Bursting Strength of Corrugated and Solid Fiberboard

Edgewise Compressive Strength of Corrugated

Fiberboard (Short Column Test)

Pin Adhesion of Corrguated Board by Selective Separation 
UNITED STATES TESTING COMPANY, INC.

CHEMICAL SERVICES DIVISION

1415 Park Avenue, Hoboken, NJ 07030

Stephen C. Pevera Phone: 201-792-2400

Accreditation Renewal Date: January 1, 1988

NYAP Code Designation Short Title

Measurements of Intrinsic Physical Properties

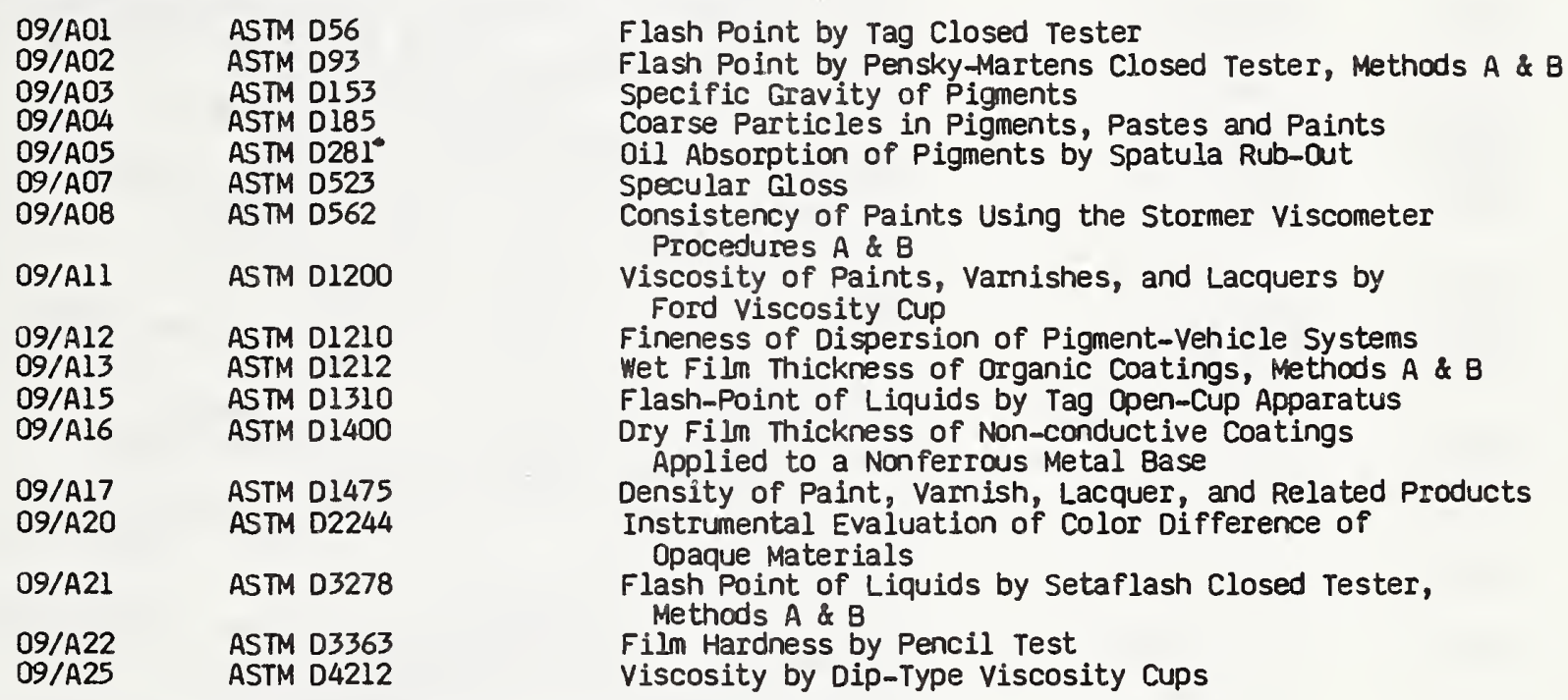

Measurements of Performance and Performance Change

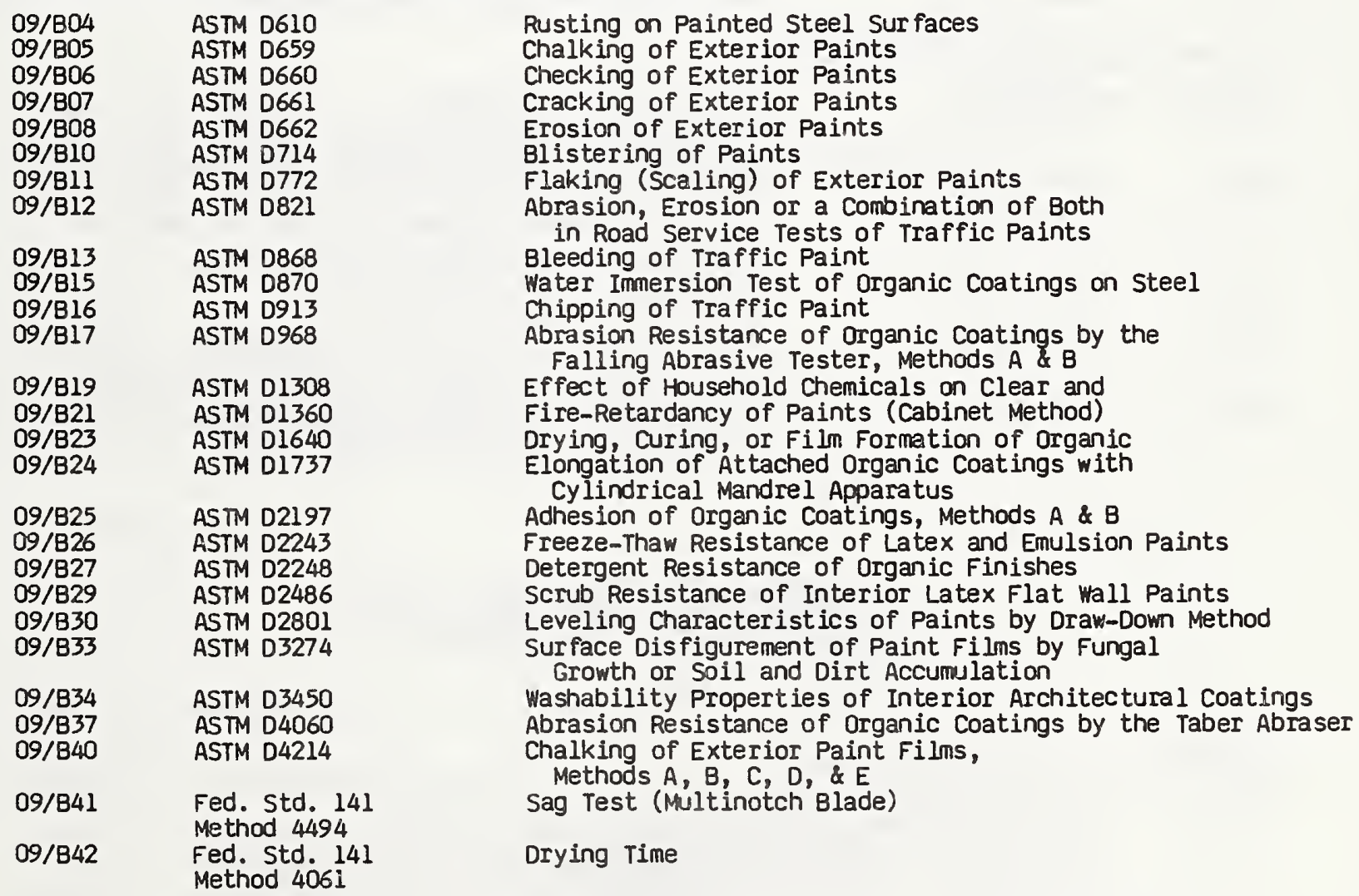




\begin{tabular}{|c|c|}
\hline 09/CO2 & ASTM D95 \\
\hline $\begin{array}{l}09 / C 06 \\
09 / C 09 \\
09 / C 11\end{array}$ & $\begin{array}{l}\text { ASTM D1078 } \\
\text { ASTM D1259 } \\
\text { ASTM D1353 }\end{array}$ \\
\hline $09 / C 12$ & ASTM D1364 \\
\hline $09 / C 15$ & ASTM D1398 \\
\hline $\begin{array}{l}09 / C 19 \\
09 / C 20\end{array}$ & $\begin{array}{l}\text { ASTM D1541 } \\
\text { ASTM D1613 }\end{array}$ \\
\hline $\begin{array}{l}09 / C 21 \\
09 / C 22 \\
09 / C 23 \\
09 / C 24 \\
09 / C 25\end{array}$ & $\begin{array}{l}\text { ASTM D1639 } \\
\text { ASTM D1644 } \\
\text { ASTM D1652 } \\
\text { ASTM D2075 } \\
\text { ASTM D2076 }\end{array}$ \\
\hline $\begin{array}{l}09 / C 26 \\
09 / C 27 \\
09 / C 28 \\
09 / C 29\end{array}$ & $\begin{array}{l}\text { ASTM D2369 } \\
\text { ASTM D2371 } \\
\text { ASTM D2697 } \\
\text { ASTM D2698 }\end{array}$ \\
\hline $\begin{array}{l}09 / C 31 \\
09 / C 32\end{array}$ & $\begin{array}{l}\text { ASTM D3009 } \\
\text { ASTM D } 3271\end{array}$ \\
\hline $09 / C 33$ & ASTM D 3272 \\
\hline $09 / C 37$ & ASTM D3723 \\
\hline $09 / C 38$ & ASTM D3792 \\
\hline $09 / C 39$ & ASTM D3960 \\
\hline $09 / C 40$ & ASTM D4017 \\
\hline
\end{tabular}

Water in Petroleum Products and Bituminous Materials by Distillation

Distillation Range of Volatile Organic Liquids

Nonvolatile content of Resin Solutions, Methods A \& $B$

Nonvolatile Matter in volatile Solvents for Use in Paint, Varnish, Lacquer and Related Products

Water in Volatile Solvents (Fischer Reagent Titration Method)

Fatty Acid Content of Alkyd Resins and Alkyd Resin Solutions, Methods A \& B

Total Iodine Value of Drying 0ils and Their Derivatives Acidity in Volatile Solvents and Chemical Intermediates Used in Paint, Vamish, Lacquer and Related Products Acid Value of Organic Coating Materials

Norivolatile Content of Vamishes, Methods $A \& B$

Epoxy Content of Epoxy Resins

Iodine Value of Fatty Amines, Amidoamines, and Diamines

Acid Value and Amine Value of Fatty Quatemary Amnonium Chlorides

Volatile Content of Paints, Procedures A \& B

Pigment Content of Solvent-Type Paints

Volume Nonvolatile Matter in Clear or Pigmented Coatings

Pigment Content of Solvent-Type Paints by High-Speed Centrifuging

composition of Turpentine by Gas Chromatography

Direct Injection of Solvent-Base Paints into a Gas Chromatograph for Solvent Analysis

vacuum Distillation of Solvents from solvent-Base Paints for Analysis

Pigment Content of Water-Emulsion Paints by Low-Temperature Ashing

Water Content of Waterborne Paints by Direct Injection into a Gas Chromatograph

Volatile Organic Contents (VOC) of Paints and Related coatings

Water in Paints and Paint Materials by Karl Fischer Method

Test Sample Conditioning and Preparation

$\begin{array}{ll}09 / 001 & \text { ASTM B117 } \\ 09 / 002 & \text { ASTM D609 } \\ 09 / D 04 & \text { ASTM D823 } \\ 09 / 007 & \text { ASTM D } 654 \\ 09 / 008 & \text { ASTM D } 1730 \\ 09 / 011 & \text { ASTM D2372 } \\ 09 / 013 & \text { ASTM D3924 } \\ 09 / D 16 & \text { ASTM G53 }\end{array}$

Salt Spray (Fog) Testing

Preparation of Steel Panels for Testing Paints, Varnish, Lacquer, and Related Products, Methods A, B, C, \& D

Producing Films of Uniform Thickness of Paint, Varnish, Lacquer, and Related Products on Test Panels, Method B

Painted or Coated Specimens Subjected to Corrosive Environments, Procedures $A$ \& B

Preparation of Aluminum and Aluminum-Alloy

Surfaces for Painting, Types $A$ \& $B$

Separation of Vehicle Solvent-Type Paints

Standard Environment for Conditioning and Testing

Paint, Vamish, Lacquer, and Related Materials

operating Light and Water-Exposure Apparatus (Fluorescent

UV-Condensation Type) for Exposure of Nonmetallic Materials

NVLAP LAB COOE 0267

RETLIF, INC. TESTING LABORATORIES

795 Marconi Avenue, Ronkonkoma, NY 11779

Walter A. Poggi Phone: 516-737-1500

Accreditation Renewal Date: October 1, 1987

NVLAP Test

Method Code Test Method Designation

12/CO1 Conducted Emissions, Power Lines, $450 \mathrm{KHz}$ to $30 \mathrm{MHz}$

FCC Method - 47 CFR Part 15 Subpart J

12/RO1 Radiated Emissions, $30 \mathrm{MHz}$ to $1000 \mathrm{MHz}$

FCC Method - 47 CFR Part 15 Subpart J 
12/TO1 Terminal Equipment Compatibility

FCC Method - 47 CFR Part 68 Subpart D

68.302 Environmental simulation, Para. c, $d, e, f$

68.304 Leakage current limitations

68.306 Hazardous voltage limitations

68. 308 Signal power limitations

68.310 Longitudinal balance limitations

68.312 on-hook impedance 1 imitations

68.314 Billing protection

12/TO2 Terminal Equipment Compatibility

FCC Method - 47 CFR Part 68 Subpart D

68.316 Hearing aid compatibility: technical standards

12/T03 Terminal Equipment Compatibility

FCC Method - 47 CFR Part 68 Subpart D

68.302 Environmental simulation, Para. a, b

EMACO, INC.

7562 Trade Street, San Diego, CA 92121

Herbert K. Mertel Phone 619-578-1480

Accreditation Renewal Date: October 1, 1987

NRAP Test

Method Code Test Method Designation

12/COl Conducted Emissions, Power Lines, $450 \mathrm{KHz}$ to $30 \mathrm{MHz}$

FCC Method - 47 CFR Part 15 Subpart J

12/RO1 Radiated Emissions, $30 \mathrm{MHz}$ to $1000 \mathrm{MHz}$

FCC Method - 47 CFR Part 15 subpart J

NORAND CORPORATION

550 Second Street, S.E., Cedar Rapids, IA

Michael W. Howard Phone: 319-369-3539

Accreditation Renewal Date: October 1, 1987

NVLAP Test

Method Code Test Method Designation

12/COl Conducted Emissions, Power Lines, $450 \mathrm{KHz}$ to $30 \mathrm{MHz}$

FCC Method - 47 CFR Part 15 Subpart J

12/RO1 Radiated Emissions, $30 \mathrm{MHz}$ to $1000 \mathrm{MHz}$

FCC Method - 47 CFR Part 15 Subpart J

NVLAP LAB CODE 0269

NVLAP LAB CODE 0270

DASH, STRAUS, \& GOODHUE, INC.

593 Massachusetts Avenue, Boxborough, MA 01719

Glen Dash Phone: 617-263-2662

Accreditation Renewal Date: October 1, 1987

NVLAP Test

Method Code Test Method Designation

12/COl Conducted Emissions, Power Lines, $450 \mathrm{KHz}$ to $30 \mathrm{MHz}$

12/RO1 FCC Method - 47 CFR Part 15 Suopart J

FCC Method - 47 CFR Part 15 Subpart J 
$12 /$ TOL

Terminal Equipment Compatibility

FCC Method - 47 CFR Part 68 Subpart D

68.302 Environmental simulation, Para. $c, d, e, f$

68.304 Leakage current limitations

68.306 Hazardous voltage limitations

68.308 Signal power limitations

68.310 Longitudinal balance Iimitations

68.312 On-hook impedance limitations

68.314 Billing protection

12/T02 Terminal Equipment Compatibility

FCC Method - 47 CFR Part 68 Subpart D

68.316 Hearing aid compatibility: technical standards

NVLAP LAB CODE 0271

AMAOOR CORPORATION

Wild Mountain Road, Almelund, MN 55002

Daniel D. Hoolihan Phone: 612-583-3322

Accreditation Renewal Date: October 1, 1987

NVLAP Test

Method Code Test Method Designation

12/ COl Conducted Emissions, Power Lines, $450 \mathrm{KHz}$ to $30 \mathrm{MHz}$

FCC Method - 47 CFR Part 15 subpart J

12/RO1 Radiated Emissions, $30 \mathrm{MHz}$ to $1000 \mathrm{MHz}$

FCC Method - 47 CFR Part 15 Subpart J

12/TO1 Terminal Equipment Compatibility

FCC Method - 47 CFR Part 68 Subart D

68.302 Environmental simulation, Para. c, d, e, $f$

68.304 Leakage current limitations

68.306 Hazardous vol tage limitations

68.308 Signal power limitations

68.310 Longitudinal balance limitations

68.312 on-hook impedance limitations

68.314 Billing protection

12/T02 Terminal Equipment Compatibility

FCC Method - 47 CFR Part 68 Subpart D

68.316 Hearing aid compatibility: technical standards

NVLAP LAB COOE 0272

COMMNICATION CERTIFICATION LABORATORY

1940 west Alexander Street, Salt Lake City, UT 84119

Thomas C. Jackson Phone: 801-972-6146

Accreditation Renewal Date: October 1, 1987

NVLAP TESt

Method Code Test Method Designation

12/CO1 Conducted Emissions, Power Lines, $450 \mathrm{KHz}$ to $30 \mathrm{MHz}$

FCC Method - 47 CFR Part 15 Subpart J

12/RO1 Radiated Emissions, $30 \mathrm{MHz}$ to $1000 \mathrm{MHZ}$

FCC Method - 47 CFR Part 15 Subpart J

12/T01 Terminal Equipment Compatibility

FCC Method - 47 CFR Part 68 Subpart D

68.302 Environmental simulation, Para. c, d, e, $f$

68.304 Leakage current limitations

68.306 Hazardous voltage limitations 
68.308 Signal power limitations

68.310 Longitudinal balance limitations

68.312 on-hook impedance limitations

68.314 Billing protection

12/T02 Terminal Equipment Compatibility

FCC Method - 47 CFR Part 68 Subpart D

68.316 Hearing aid compatibility: technical standards

12/T03 Terminal Equipment compatibility

FCC Method - 47 CFR Part 68 Subpart D

68.302 Environmental simulation, Para. a, b

MET ELECTRICAL TESTING COMPANY, INC.

916 West Patapsco Avenue, Baltimore, MD 21230

Leonard Frier Phone: 301-354-2200

Accreditation Renewal Date: October 1, 1987

MLLAP Test

Method Code

$12 / \mathrm{COI}$

Test Method Designation

12/ROI

Conducted Emissions, Power Lines, $450 \mathrm{KHz}$ to $30 \mathrm{MHz}$

FCC Method - 47 CFR Part 15 subpart J

Radiated Emissions, $30 \mathrm{MHz}$ to $1000 \mathrm{MHz}$

FCC Method - 47 CFR Part 15 subpart J

12/TO1 Terminal Equipment Compatibility

FCC Method - 47 CFR Part 68 subpart D

68.302 Enviromental simulation, Para. c, d, e, $f$

68.304 Leakage current limitations

68.306 Hazardous voltage limitations

68.308 Signal power limitations

68.310 Longitudinal balance limitations

68.312 on-hook impedance limitations

68.314 Billing protection

12/T02 Terminal Equipment Compatibility

FCC Method - 47 CFR Part 68 Subpart D

68.316 Hearing aid compatibility: technical standards

12/T03 Terminal Equipment Compatibility

FCC Method - 47 CFR Part 68 Subpart D

68.302 Environmental simulation, Para. a, b

NVLAP LAB COOE 0274

GTE EVALUATION \& SUPPORT DEPARTMENT

3050 Harrodsburg Road, Lexington, KY 40503

Clifford Eugene Jones Phone: 606-223-3061

Accreditation Renewal Date: October 1, 1987

NVLAP Test

Method Code

Test Method Designation

$12 / \mathrm{COl}$

Conducted Emissions, Power Lines, $450 \mathrm{KHz}$ to $30 \mathrm{MHz}$

FCC Method - 47 CFR Part 15 subpart J

12/RO1 Radiated Emissions, $30 \mathrm{MHz}$ to $1000 \mathrm{MHz}$

FCC Method - 47 CFR Part 15 Subpart J

12/TO1 Terminal Equipment Compatibility

FCC Method - 47 CFR Part 68 Subpart D

68.302 Environmental simulation, Para. c, d, e, f

68.304 Leakage current limitations

68.306 Hazardous voltage limitations 
68.308 Signal power limitations

68.310 Longitudinal balance limitations

68.312 on-hook impedance limitations

68.314 Billing protection

12/T02 Terminal Equipment Compatibility

FCC Method - 47 CFR Part 68 Subpart D

68.316 Hearing aid compatibility: technical standards

12/T03 Terminal Equipment Compatibility

FCC Method - 47 CFR Part 68 Subpart D

68.302 Environmental simulation, Para. a, b

NVLAP LAB CODE 0275

AT \& T INFORMATION SYSTEMS

EMC LABORATORY

MS: Building 41-112, Crawfords Corner Road, Holmdel, NJ 07733 D.N. Heirman Phone: 201-834-3566

Accreditation Renewal Date: October 1, 1987

NVLAP Test

Method Code Test Method Designation

12/CO1 Conducted Emissions, Power Lines, $450 \mathrm{KHz}$ to $30 \mathrm{MHz}$

FCC Method - 47 CFR Part 15 Subpart J

12/RO1 Radiated Emissions, $30 \mathrm{MHz}$ to $1000 \mathrm{MHz}$

FCC Method - 47 CFR Part 15 Subpart J

NMAP LAB CODE 0276

D.L.S. ELECTRONIC SYSTEMS, INC.

10350 Dearlove Road, Glenview, IL 60025

Donald L. Sweeney Phone: 312-699-9060

Accreditation Renewal Date: October 1, 1987

NVLAP Test

Method Code Test Method Designation

12/CO1 Conducted Emissions, Power Lines, $450 \mathrm{KHz}$ to $30 \mathrm{MHz}$

FCC Method - 47 CFR Part 15 Subpart J

12/RO1 Radiated Emissions, $30 \mathrm{MHz}$ to $1000 \mathrm{MHz}$

FCC Method - 47 CFR Part 15 Subpart J

NVLAP LAB COOE 0277

CONTINENTAL TESTING LABORATORIES

8385 South U.S. Highway 17-92, Fern Park, FL 3730-2898

Chester A. Mitchell Phone: 305-831-2700

Accreditation Renewal Date: October 1, 1987

NVLAP TEST

Method Code Test Method Designation

12/CO1 Conducted Emissions, Power Lines, $450 \mathrm{KHz}$ to $30 \mathrm{MHz}$

FCC Method - 47 CFR Part 15 Subpart J

Radiated Enissions, $30 \mathrm{MHz}$ to $1000 \mathrm{MHz}$

12/TO1 TCC Method - 47 CFR Part 15 Subp

FCC Method - 47 CFR Part 68 Subpart D

68.302 Environmental simulation, Para. c, d, e, f

68.304 Leakage current limitations

68.306 Hazardous voltage limitations 
68.308 Signal power limitations

68.310 Longitudinal balance limitations

68.312 on-hook impedance limitations

68.314 Billing protection

12/T02 Terminal Equipment Compatibility

FCC Method - 47 CFR Part 68 Subpart D

68.316 hearing aid compatibility: technical standards

12/T03 Terminal Equipment Compatibility

FCC Method - 47 CFR Part 68 Subpart D

68.302 Environmental simulation, Para. a, b

NVLAP LAB CODE 0278

\author{
ELITE ELECTRONIC ENGINEERING COMPANY \\ 1516 Centre Circle, Downers Grove, IL 60515 \\ James C. Klouda Phone: 312-495-9700
}

Accreditation Renewal Date: October 1, 1987

NVLAP Test

Method Code

Test Method Designation

$12 / \mathrm{COl}$

Conducted Emissions, Power Lines, $450 \mathrm{KHz}$ to $30 \mathrm{MHz}$

FCC Method - 47 CFR Part 15 Subpart J

12/RO1

Radiated Emissions, $30 \mathrm{MHz}$ to $1000 \mathrm{MHz}$

FCC Method - 47 CFR Part 15 Subpart J

12/T01 Terminal Equipment Compatibility

FCC Method - 47 CFR Part 68 Subpart D

68.302 Environmental simulation, Para. c, d, e, $f$

68.304 Leakage current limitations

68.306 Hazardous voltage limitations

68.308 Signal power limitations

68.310 Longitudinal balance limitations

68.312 on-hook impedance limitations

68.314 Billing protection

12/T02 Terminal Equipment Compatibility

FCC Method - 47 CFR Part 68 Subpart D

68.316 Hearing aid compatibility: technical standards

12/T03 Terminal Equipment Compatibility

FCC Method - 47 CFR Part 68 Suopart D

68.302 Environmental simulation, Para, a, b

NVLAP LAB COOE 0279

PHILIPPINE GEOANALYTICS

No. 3 Scout Magbanua

Quezon City, Metro Manila, Philippines

Emilio M. Morales Telex: 66156 HDADB PN

Accreditation Renewal Date: July 1, 1987

NVLAP Code Designation

Short Title

02/GO1 ASTM C31

ASTM $\mathrm{Cl} 72$

ASTM C143

ASTM Cl38

ASTM C231

Making and Curing Test Specimens

Sampling Freshly Mixed concrete

Slump of Port land Cement Concrete

Unit Weight, Yield, and Air Content

Air Content-Pressure Method

O2/AO1

ASTM C 39

Compressive Strength of Cylindrical Specimens 
$R$ \& B ENTERPRISES

20 Clipper Road West Conshohocken, PA 19428,

Finbarr M. O'Connor, Jr. Phone: 215-825-1960

Accreditation Renewal Date: October 1, 1987

NVLAP Test

Method Code

12/COl Conducted Emissions, Power Lines, $450 \mathrm{KHz}$ to $30 \mathrm{MHz}$

FCC Method - 47 CFR Part 15 Subpart J

12/RO1 Radiated Emissions, $30 \mathrm{MHz}$ to $1000 \mathrm{MHz}$

12/TO1

12/T02
FCC Method - 47 CFR Part 15 Subpart J

Terminal Equipment Compatibility

FCC Method - 47 CFR Part 68 Subpart D

68.302 Environmental simulation, Para. c, d, e, $f$

68.304 Leakage current limitations

68.306 Hazardous vol tage limitations

68.308 Signal power limitations

68.310 Longitudinal balance limitations

68.312 on-hook impedance limitations

68.314 Billing protection

Terminal Equipment Compatibility

FCC Method - 47 CFR Part 68 Subpart D

68.316 Hearing aid compatibility: technical standards

NVLAP LAB COOE 0281

STANDARD T CHEMICAL TECHNICAL CENTER

10th \& Washington Streets Chicago Heights, IL 60411 Mr. L.G. Hering 312-755-1223

Accreditation Renewal Date: October 1, 1987

NVLAP Test Method

Code Designation

Short Title

Measurements of Intrinsic Physical Properties

$\begin{array}{ll}\text { 09/A01 } & \text { ASTM D56 } \\ \text { 09/A07 } & \text { ASTM D523 } \\ \text { 09/A08 } & \text { ASTM D562 } \\ & \\ 09 / A 12 & \text { ASTM D } 1210 \\ \text { 09/A15 } & \text { ASTM D } 1310 \\ 09 / A 17 & \text { ASTM D1475 } \\ 09 / \text { A26 } & \text { ASTM E97 } \\ \text { 09/A28 } & \text { ASTM E313 }\end{array}$

Flash Point by Tag Closed Tester

Specular Gloss

Consistency of Paints Using the Stormer Viscometer Procedures $A$ \& $B$

Fineness of Dispersion of Pigment-Vehicle Systems

Flash-Point of Liquids by Tag Open-Cup Apparatus

Density of Paint, Varnish, Lacquer, and Related Products

45- deg, O-deg Directional Reflectance Factor of Opaque Specimens by Broad-Band Filter Reflectometry

Indexes of whiteness and Yellowness of Near-White opaque Materials

Measurements of Performance and Performance Change

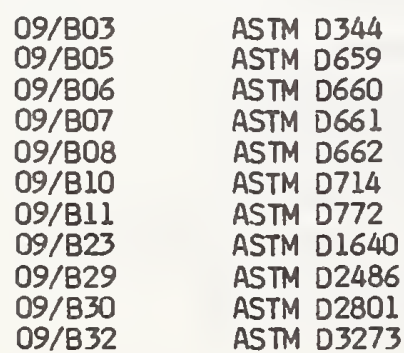

Relat ive Dry Hiding Power of Paints

Chalking of Exterior Paints

Checking of Exterior Paints

Cracking of Exterior Paints

Erosion of Exterior Paints

Blistering of Paints

Flaking (Scaling) of Exterior paints

Drying, Curing, or Film Formation of Organic

Scrub Resistance of Interior Latex Flat Wall paints

Leveling Characteristics of Paints by Draw-Down Method

Resistance to Growth of Mold on the Surface of

Interior Coatings in an Environmental Chamber 
09/B33 ASTM D 3274

$09 / B 34$

$09 / B 37$

$09 / B 38$

$09 / 840$

$09 / 841$
ASTM D3450

ASTM D4060

ASTM D4062

ASTM D4214

Fed. Std. 141

Method 4494
Surface Disfigurement of Paint Films by Fungal

Growth or Soil and Dirt Accumulation

Washability Properties of Interior Architectural Coatings

Abrasion Resistance of Organic Coatings by the Taber Abraser Leveling of Paints by Draw-Down Method

Chalking of Exterior Paint Films

Sag Test (Multinotch Blade)

NLLAP LAB CODE 0501

BALTIMORE GAS \& ELECTRIC COMPANY, CALVERT CLIFFS NUCLEAR POWER PLANT

NUCLEAR POWER DEPARTMENT, DOSIMETRY UNIT

RADIATION SAFETY SECTION

Lusby, MD 20657

- Eugene T. Reimer Phone: 301-269-4716

Accreditation Renewal Date: October 1, 1988

This facility has been evaluated and deemed competent to process the radiation dosimeter listed below through employing a Panasonic Automatic reader model UD71OA and Panasonic Manual reader UD702A.

This facility is accredited to process the following dosimeter by virtue of actual demonstration of compliance with ANSI-N13.11-1983 through testing.

Panasonic RD model UD802 for ANSI-N13.11 categories II, IV, V, VII, VIII.

NVLAP LAB CODE 0502

\author{
UNION ELECTRIC COMPANY \\ CALLAWAY PLANT \\ P.0. Box 620, Fulton, MO 65251 \\ Ron Roselius Phone: 314-676-8321
}

Accreditation Renewal Date: April 1, 1987

This facility has been evaluated and deemed competent to process the radiation dosimeter listed below through employing a Panasonic Automatic reader model UD71OA and Panasonic Manual reader UD702E.

This facility is accredited to process the following dosimeter by virtue of actual demonstration of compliance with ANSI-N13.11-1983 through testing.

Panasonic TLD model UD802 for ANSI-N13.11 categories II, VI, VII, VIII.

NVLAP LAB CODE 0503

MALLINCKRODT DIAGNDSTICS, INC.

2703 Wagner Place, Maryland Heights, MO 63043

LeeAnn Borcherding Phone: 314-344-3981

Accreditation Renewal Date: October 1, 1988

This facility has been evaluated and deemed competent to process the radiation dosimeter listed below through employing Harshaw Automatic readers model $2000 \mathrm{~B}$ and 20000.

This facility is accredited to process the following dosimeter by virtue of actual demonstration of compliance with ANSI-N13.11-1983 through testing.

Harshaw TLD model 100 for ANSI-N13.11 category VII. 


\author{
NAVAL MEDICAL COMMAND \\ NATIONAL CAPITAL REGION \\ RADIATION SAFETY OEPARTMENT \\ Bethesda, MD 20814 \\ Robert T. Devine Phone: 202-295-5414
}

Accreditation Renewal Date: October 1, 1988

This facility has been evaluated and deemed competent to process the radiation dosimeters listed below through employing a Harshaw Automatic reader model 2271 and Manual film processing using a Macbeth densitometer.

This facility is accredited to process the following dosimeter by virtue of actual demonstration of compllance with ANSI-N13.11-1983 through testing.

Harshaw TLO Albedo ( 1 TLD 600, 1 TLD 700) for ANSI-N13.11 categories II, IV, VIII.

F1Im Badge (Kodak Type 3) for ANSI-N13.11 Categorles III, IV, V, VI, VII.

NVAP LAB CODE 0505

DUKE POWER COMPANY, DOSIMETRY LABORATORY

Physical Sciences Building

Route 4, Box 531, Huntersville, NC 28078

Wanda M. Carter Phone: 704-875-1971

Accreditation Renewal Date: April 1, 1987

This facility has been evaluated and deemed competent to process the radiation dosimeter listed below through employing Teledyne Automatic readers model 9100 and 9150 , and Teledyne Manual readers model 8300 and 8310 .

This facility is accredited to process the following dosimeter by virtue of actual demonstration of compliance with ANSI-N13.11-1983 through testing.

Teledyne TLD model BP3 for ANSI-N13.11 categorles II, IV, V, VII.

NVLAP LAB COOE 0506

\author{
SOUTHERN CALIFORNIA EDISON \\ SAN ONDFRE NUCLEAR GENERATING STATION \\ P.0. Box 128, San Clemente, CA 92672 \\ Robert Dickey Phone: 714-368-6254
}

Accreditation Renewal Date: October 1, 1988

This facility has been evaluated and deemed competent to process the radiation dosimeter listed below through employing a Panasonic Automatic reader model UD710A.

This facility is accredited to process the following dosimeter by virtue of actual demonstration of compliance with ANSI-N13.11-1983 through testing.

Panasonic TLD model W0802-AS2 for ANSI-N13.11 categorles I, II, III, IV, V, VI, VII. 


\author{
NEW YORK POWER AUTHORITY \\ INDIAN POINT UNIT NO. 3 NUCLEAR POWER PLANT \\ $P .0$. Box 215, Buchanan, NY 10511 \\ Thomas Labenski Phone: 914-739-8200
}

Accreditation Renewal Date: October 1, 1988

This facility has been evaluated and deemed competent to process the radiation dosimeter listed below through employing a Panasonic Automatic reader model UD710B and Panasonic Manual reader UD702E.

This facility is accredited to process the following dosimeter by virtue of actual demonstration of compliance with ANSI-N13.11-1983 through testing.

Panasonic TLD model UD806AQ for ANSI-N13.11 categories I, II, III, IV, V, VI, VII.

NVLAP LAB CODE 0509

\author{
NAVAL RESEARCH LABORATORY \\ Code 4073, Washington, DC 20375 \\ Kirk J. King Phone: 202-767-2232
}

Accreditation Renewal Date: January 1, 1989

This facility has been evaluated and deemed competent to process the radiation dosimeter listed below through employing a Harshaw Automatic reader model 2271.

This facility is accredited to process the following dosimeter by virtue of actual demonstration of compliance with ANSI-N13.11-1983 through testing.

NRL Radiation Badge for ANSI-N13.11 categories II, III, IV, V, VI, VII, VIII.

NVLAP LAB CODE 0510

GENERAL PUBLIC UTILITIES NUCLEAR CORPORATION

OIVISION OF RADIOLOGICAL \& ENVIRONMENTAL CONTROLS

Route 441 South, P.O. Box 480, Middletown, PA 17057

0 . Ronald Perry Phone: 717-948-8595

Accreditation Renewal Date: October 1, 1988

This facility has been evaluated and deemed competent to process the radiation dosimeter listed below through employing a Panasonic Automatic reader model 40710.

This facility is accredited to process the following dosimeter by virtue of actual demonstration of compliance with ANSI-N13.11-1983 through testing.

Panasonic TLD model UD802-2 for ANSI-N13.11 categories I, II, III, IV, V, VI, VII, and Panasonic TLD model UD802-2N for ANSI-N13.11 categories IV, VIII.

NVLAP LAB COOE 0511

\author{
NEW YORK POWER AUTHORITY \\ JAMES A. FITZPATRICK NUCLEAR POWER PLANT \\ P.0. Box 41, Lycoming, NY 13093 \\ MI. George J. Vargo Phone: 315-342-3840
}

Accreditation Renewal Date: Cctober 1, 1988

This facility has been evaluated and deemed competent to process the radiation dosimeter listed below through employing a Panasonic Automatic reader model UD710A.

This facility is accredited to process the following dosimeter by virtue of actual demonstration of compliance with ANSI-N13.11-1983 through testing.

Panasonic TLD model UD812 for ANSI-N13.11 categories II, IV, VI, VII. 


\title{
RADIATION DETECTION COMPANY \\ 162 Wolfe Road, P.O. Box 1414, Sunnyvale, CA 94088 \\ Richard H. Holden Phone: 408-735-8700
}

Accreditation Renewal Date: October 1, 1988

This facility has been evaluated and deemed competent to process the radiation dosimeters listed below through employing (1) modified CON RAD readers; (2) Teledyne 7100 reader; (3) Teledyne 7300 reader; (4) Harshaw 3000 reader; (5) Victoreen 2800 reader; (6) by manual film processing and reading on a Macbeth TD502 densitometer; or (7) Tracketch, NTA manual optical readers.

This facility is accredited to process the following dosimeters by virtue of actual demonstration of compliance with ANSI-N13.11-1983 through testing.

\section{Designation Process}

$\begin{array}{ll}\text { Hi Energy TLD } & 1 \\ \text { Beta TLD } & 1,3 * \\ \text { Lo Energy TLD } & 1,3 * \\ \text { TLD Albedo } & 3 *, 6 \\ \text { Film XBG } & 6 \\ \text { Film XBGN } & 6,7 \\ \text { Neutron Tracketch }\end{array}$

\section{ANSI N13.11 Categories}

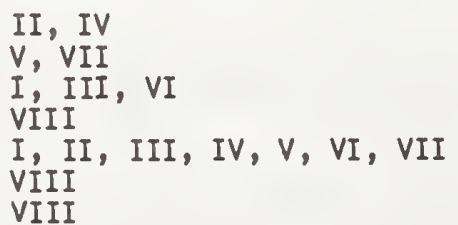

* Processes listed above 2, 4, and 5 are considered functionally acceptable as substitutes which can be used in lieu of process 3 as listed above.

NVLAP LAB COOE 0513

QULF NUCLEAR, INC.

$100 \mathrm{E}$. Nasa Road One, 非11, Webster, TX 77598

Joseph Pryber Phone: 713-338-2652

Accreditation Renewal Date: October 1, 1988

This facility has been evaluated and deemed competent to process the radiation dosimeter listed below through employing a Teledyne Automatic reader mcdel 9150.

This facility is accredited to process the following dosimeter by virtue of actual demonstration of compliance with ANSI-N13.11-1983 through testing.

Teledyne TLD model PB3 loaded with a RGD-CaSO4:Dy-0.4 B/G card and a RGD-6LiF/CaSO4:Dy-0.4 Neutron card for ANSI-13.11 categories I, II, III, IV, V, VI, VII, and VIII.

NVLAP LAB COOE 0514

\author{
ROCHESTER GAS \& ELECTRIC CORP. \\ R.E. GINNA NUCLEAR POWER PLANT \\ 1503 Lake Road, Ontario, NY 14519 \\ Bernard R. Quinn Phone: 315-524-4446
}

Accreditation Renewal Date: october 1, 1987

This facility has been evaluated and deemed competent to process the radiation dosimeter listed below through employing a Panasonic Automatic reader model UD710A and Panasonic Manual reader UD702A..

This facility is accredited to process the following dosimeter by virtue of actual demonstration of compliance with ANSI-N13.11-1983 through testing.

Panasonic TLD model UD802 for ANSI-N13.11 categories I, II, III, IV, V, VI, VII, VIII. 


\author{
THERMO ANALYTICAL INC. \\ TMAVEBERLINE \\ 5635 Kircher Boulevard NE \\ Post Office Box 3874 \\ Albuquerue, MM 87190-3874 \\ Nels Johnson Phone: 505-345-9931
}

Accreditation Renewal Date: October 1, 1988

This facility has been evaluated and deemed competent to process the radiation dosimeter listed below through employing a Eberline Manual reader TLR-6.

This facility is accredited to process the following dosimeters by virtue of actual demonstration of compliance with ANSI-N13.11-1983 through testing.

Eberline TLD (2 or 3 Harshaw TLD 100 chips) for ANSI-N13.11 categories I, II, III, IV, V, VI, VII, VIII.

Eberline Albedo (4 TLD100 chips) for ANSI-N13.1l Category VIII.

NVLAP LAB COOE 0516

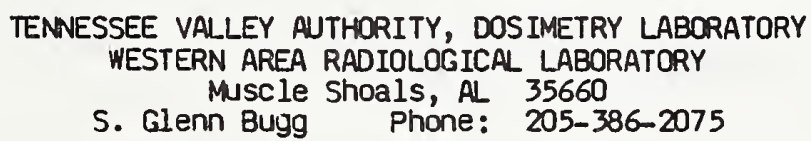

Accreditation Renewal Date: April 1, 1987

This facility has been evaluated and deemed competent to process the radiation dosimeters listed below through employing a Panasonic Automat ic reader model UD71OA and Panasonic Manual reader UD702A.

This facility is accredited to process the following dosimeter by virtue of actual demonstration of compliance with ANSI-N13.11-1983 through testing.

Panasonic TLD model UD802 for ANSI-N13.11 categories I, II, III, IV, V, VI, VII, VIII.

The following sites are included in the accreditation as sub-facilities of the above listed main facility and are accredited for the same equipment and dosimeter listed.

Browns Ferry Nuclear Plant, Decatur, Alabama

Watts Bar Nuclear Plant, Spring City, Tennessee

Sequoyah Nuclear Plant, Daisy, Tennessee

\title{
NVLAP LAB COOE 0517
}

CAROLINA POWER \& LIGHT COMPANY

HARR IS ENERGY \& ENVIRONMENTAL CENTER

Route 1, Box 327, New Hill, NC 27562

Stephen A. Browne Phone: 919-362-3212

Accreditation Renewal Date: October 1, 1988

This facility has been evaluated and deemed competent to process the radiation dosimeter listed below through employing a Panasonic Automatic reader model UD710A and Panasonic Manual reader UD702E.

This facility is accredited to process the following dosimeter by virtue of actual demonstration of compliance with ANSI-N13.11-1983 through testing.

Panasonic TL model uD802 for ANSI-N13.11 categories I, II, III, IV, V, VI, VII, VIII. 
The following sites are insluded in the accreditation as sub-facilities of the above listed main facility. These sub-facilities are accredited by virtue of using identical equipment and procedures as indicated above.

Robinson Nuclear Plant, Hartsville, South Carolina Brunswick Nuclear Plant, Southoort, North Carolina Harris Nuclear Project, New Hill, North Carolina

NVLAP LAB CODE 0518

R.S. LANDAUER JR. \& COMPANY

Glenwood Science Park, 2 Science Park, Glenwood, IL 60425 Craig Yoder Phone: 312-755-7000

Accreditation Renewal Date: October 1, 1988

This facility has been evaluated and deemed competent to process the radiation dosimeters listed below through employing (1) autonatic film reader Tech/0ps model 1; (2) Harshaw Atlas Hotgas reader; (3) Harshaw 2271 reader; (4) NTA/Polycarbonate /CR-39 manual optical readers; or (5) manual densitometers X-Rite, Tech/Ops model 301, Macbeth model TD504.

This facility is accredited to process the following dosimeter by virtue of actual demonstration of compliance with ANSI-N13.11-1983 through testing.

Landauer designation

Film

G - FIIm "GARDRAY"

P - G badge plus NTA

A - $G$ badge plus polycarbonate

TLD

L - 4 chip "GARDRAY"

D - 3700 chip Harshaw card

I - Neutrak ER

$T$ modified - 3 100/700 chips
Process ANSI N13.11 Category

$\begin{array}{ll}1,5 & I, I I, \text { III, IV, V, VI, VII } \\ 1,4,5 & \text { VIIII } \\ 1,4 & \text { VIII }\end{array}$

2 I, II, III, IV, V, VI, VII

3 II, IV, V, VII

3,4 VIII

2 I, II, III, IV, V, VI, VII

The facility is accredited to process the following dosimeters wich have been deemed functionally acceptable by virtue of using identical techniques and equipment to process combinations of elements demonstrated above.

Landauer designation

Film

$B$ - G badge plus CR-39

$C$ - $G$ badge plus CR-39 and Cadmium

$P$ - $G$ badge plus NTA

$H$ - G badge plus NTA and Cadmium

A - $G$ badge plus polycarbonate

$J$ - G badge plus polycarbonate and Cadmium

$Y$ - $G$ badge plus Cadmium

$R$ - G badge plus ER

Q - DEX-RAY

TLD

F - L badge plus CR-39

- L badge plus polycarbonate

- L badge plus ER

$T=2$ chip

\section{Process ANSI N13.11 Category}

$1,4,5$ I through VIII

$1,4,5$ I through VIII

$1,4,5$ I, II, III, IV, V, VI, VII, VIII

$1,4,5$ I through VIII

$1,4,5$ I, II, III, IV, V, VI, VII, VIII

$1,4,5$ I through VII

$1,4,5$ I, III

$I, 3,4,5 \quad I, I I, I I I, I V, V, V I, V I I, V I I I$

$1,4,5$ I, III

The following sites are included in the accreditation as sub-facilities of the above listed main facility.

The following sub-facilities are accredited to process the Landauer "D" badge employing a Harshaw 2271 autonatic TLD reader for ANSI N13.11 categories II, IV, V, VII which have been deemed functionally acceptable by virtue of using identical techniques and procedures as demonstrated above for the items specified. 
R.S. Landauer, Jr. \& Company Nuclear Station System (NSS) sites at:

Boston Edison Company, Pilgrim Station, Plymouth, Massachusetts

Alabana Power, Farley Nuclear Plant, Ashford, Alabama

The following sub-facilities are accredited to perform limited volume, emergency response processing employing either a Harshaw 3000 manual reader or manual film processing techniques for the following badges:
G - Film "GARDRAY"
L - TLD 4 chIP "GARDRAY"
ANSI N13.11 Categories
ANSI N13.11 Categories
ANSI N13.11 Categories
I, II, III, IV, V, VI, VII
I, II, III, IV, V, VI, VII
II, IV, V, VII
T RD 2 chip

R. S. Landauer, Jr. \& Company Offices: El Sequndo, California; Houston, Texas; Burlington, Massachusetts; and East Brunswick, New Jersey.

The following sub-facility is accredited to process 4 Chip RD 700 (LF.) Harshaw card used with a Harshaw Type 80 Holder the Landauer NSS/PPSL.

dosimeter employing a Harshaw automatic reader type 2276 or a manual type $2000 \mathrm{~A}$ or B by virtue of actual demonstration of compliance with ANSI N13.11-1983 through testing in Categories I, II, III, IV, V, VI, VII.

Pennsylvania Power \& Light-N.S.S., 2 North Ninth Street, Allentown, PA 18101

\title{
NVLAP LAB COOE 0519
}

HOUSTON LIGHTING \& POWER COMPANY, MANAGING PARTNER

SOUTH TEXAS PROJECT ELECTRIC GENERATING STATION

P.0. Box 1700, Houston, TX 77059

Gene R. Jarvela Phone: 512-972-3651

Accreditation Renewal Date: October 1, 1988

This facility has been evaluated and deemed competent to process the radiation dosimeter listed below through employing a Panasonic Automatic reader model UD710A.

This facility is accredited to process the following dosimeter by virtue of actual demonstration of compliance with ANSI-N13.11-1983 through testing.

Panasonic RD Model UD802 for ANSI-N13.11 categories II, IV, VII.

NVLAP LAB CODE 0520

\author{
VIRGINIA ELECTRIC AND POWER COMPANY \\ NORTH ANNA POWER STATION \\ P.0. Box 402, Mineral, VA 23117 \\ Henry F. Kahnhauser Phone: 703-894-5151
}

Accreditation Renewal Date: October 1, 1988

This facility has been evaluated and deemed competent to process the radiation dosimeter listed below through employing Teledyne Automatic readers model 9100 and 9150 , and Teledyne Manual readers model 8300 and 8310 .

This facility is accredited to process the following dosimeter by virtue of actual demonstration of compliance with ANSI-N13.11-1983 through testing.

Teledyne RD model BP3 for ANSI-N13.11 categories II, IV, V, VII. 
D ' DUESNE LIGHT COMPANY

NUCLEAR OIVISION - BEAVER VALLEY FOWER STATION

P.0. Box 4, Shippingport, Pf 15077

Robert M. Vento Phone: 412-393-5722

Accreditation Renewal Date: October 1, 1987

This facility has been evaluated and deemed competent to process the radiation dosimeter listed below through employing a Panasonic Automatic reader model UD710A.

This facility is accredited to process the following dosineter by virtue of actual demonstration of compliance with ANSI-N13.11-1983 through testing.

Panasoni: TLD model UD812 for ANSI-N13.11 categorles I, II, III, IV, V, VI, VII.

NVLAP LAB COOE 0522

\author{
CONSUMERS POWER COMPANY \\ PERSONNEL DOSIMETRY LABORATORY \\ 1945 Parnall Road, Jackson, MI 49201 \\ Karl H. Andrews Phone: 517-788-0433
}

Accreditation Renewal Date: October 1, 1988

This facility has been evaluated and deemed competent to process the radiation dosimeter listed below through employing a Teledyne Automatic reader model 9100.

This facility is accredited to process the following dosimeters by virtue of actual demonstration of compliance with ANSI-N13.11-1983 through testing.

Teledyne TLD model BG for ANSI-N13.11 categories II, IV, V, VII.

Teledyne TL model BGN for ANSI-N13.11 category VIII.

NVAP LAB COOE 0523

\author{
VIRGINIA ELECTRIC \& POWER COMPANY \\ SURRY POWER STATION \\ P.0. BOX 315, Surry, VA 23883 \\ Dean Densmore Phone: 804-357-3184
}

Accreditation Renewal Date: January 1, 1989

This facility has been evaluated and deemed competent to process the radiation dosimeter listed below through employing Teledyne Automatic readers model 9100 and 9150 , and Teledyne Manual reader model 8300 .

This facility is accredited to process the following dosimeter by virtue of actual demonstration of compliance with ANSI-N13.11-1983 through testing.

Teledyne TL model PB3 for ANSI-N13.11 categor les II, IV, V, VII.

NVLAP LAB CODE 0524

YANKEE ATOMIC ELECTRIC COMPANY 1671 Worcester Road, Framingham, MA 01701 Neill Stanford Phone: 617-872-8100

Accreditation Renewal Date: october 1, 1988

This facility has been evaluated and deemed competent to process the radiation dosimeter listed below through employing a Harshaw Automatic reader model 2271.

This facility is accredited to process the following dosimeter by virtue of actual demonstration of compliance with ANSI-N13.11-1983 through testing.

Harshaw TLD model BGN for ANSI-N13.11 categories I, II, III, IV, V, VI, VII, and VIII. 
OMAHA PUBLIC POWER DISTRICT

1623 Harney Street, Omaha, NE 68102

Marilyn Hawes Phone: 402-536-4696

Accreditation Renewal Date: April 1, 1987

This facility has been evaluated and deemed competent to process the radiation dosimeter listed below through employing a Harshaw Automatic reader model $2000 \mathrm{~B}$ and a Harshaw Manual reader model 2000 .

This facility is accredited to process the following dosimeter by virtue of actual demonstration of compliance with ANSI-N13.11-1983 through testing.

Harshaw TLD model BG for ANSI-N13.11 categories II, IV, V, VII, and Harshaw TLD model GBN for ANSI-N13.11 category VIII.

NVLAP LAB CODE 0526

\author{
KANSAS GAS AND ELECTRIC COMPANY \\ WOLF CREEK GENERATING STATION \\ P.0. Box 309 , Burlington, KS 66839 \\ Larry Breshears Phone: 316-364-8831
}

Accreditation Renewal Date: January 1, 1989

This facility has been evaluated and deemed competent to process the radiation dosimeter listed below through employing a Panasonic Automatic reader model UD710A and manual reader $702 E$.

This facility is accredited to process the following dosimeter by virtue of actual demonstration of compl lance with ANSI-N13.11-1983 through testing.

Panasonic TLD model uD802 for ANSI-N13.11 categories II, III, IV, V, VI, VII, VIII.

NVLAP LAB CODE 0528

TEXAS UTILITIES GENERATING COMPANY
COMANCHE PEAK STEAM GECTRIC STATION
P.0. BOX 2300 , Glen Rose, TX 76043
John J. O'Donnell Phone: $817-897-4856$

Accreditation Renewal Date: July 1, 1987

This facility has been evaluated and deemed competent to process the radiation dosimeter listed below through employing a Panasonic Automatic reader model UD71OA and Panasonic Manual reader UD702E.

This facility is accredited to process the following dosimeter by virtue of actual demonstration of compliance with ANSI-N13.11-1983 through testing.

Panasonic TLD model UD802 for ANSI-N13.11 categories I, II, III, IV, V, VI, VII, VIII. 


\author{
DET?OIT EDISON COMPANY \\ HEAL IH PHYSICS/DOSIMETRY \\ 6400 North Dixie Highway, Newport, MI 48166 \\ Robert Koback Phone: 313-586-1037
}

Accreditation Renewal Date: October 1, 1987

This facility has been evaluated and deemed competent to process the radiation dosimeter 11 sted below through employing a Panasonic Automatic reader model UD710A and Panasonic Manual reader UD702A.

This facility is accredited to process the following dosimeter by virtue of actual demonstration of compliance with ANSI-N13.11-1983 through testing.

Panasonic TLD model 10802 for ANSI-N13.11 categories III, IV, V, VI, VII, VIII.

NVLAP LAB CODE 0530

\author{
LOUISIANA POWER AND LIGHT COMPANY \\ WATERFORO 3 STEAM ELECTRIC STATION \\ P.O. Box B, Killona, LA 70066 \\ Ronald C. McLendon Phone: 504-464-3269
}

Accreditation Renewal Date: October 1, 1987

This facility has been evaluated and deemed competent to process the radiation dosimeter listed below through employing a Panasonic Automatic reader model UD71OA and Panasonic Marual reader UD702E.

This facllity is accredited to process the following dosimeter by virtue of actual demonstration of compliance with ANSI-N13.11-1983 through testing.

Panasonic TLD model UD802 for ANSI-N13.11 categories I, II, III, IV, V, VI, VII, VIII.

NVLAP LAB CODE 0531

PUBLIC SERVICE ELECTRIC AND GAS COMPANY

NUCLEAR DEPARTMENT - RADIATION PROTECTION SERVICES

P.O. Box 236, Hancocks Bridge, NJ 08038

Jeffrey L. Kotsch Phone: 609-339-4568

Accreditation Renewal Date: October 1, 1987

This facility has been evaluated and deemed competent to process the radiation dosimeter listed below through employing a Panasonic Automatic reader model UD710.

This facility is accredited to process the following dosimeter by virtue of actual demonstration of compliance with ANSI-N13.11-1983 through testing.

Panasonic TLD model UD802 for ANSI-N13.11 categories I, II, III, IV, V, VI, VII, VIII.

NVLAP LAB CODE 0532

SIEMENS GAMMASONICS, INC.

2000 Nuclear Drive, Des Plaines, IL 60018

Robert W. Pollock Phone: 3i2-635-3396

Accreditation Renewal Date: January 1, 1988

This facility has been evaluated and deemed competent to process the radiation dosimeter listed below through employing a Harshaw Atlas reader and Manual film processing using a custom densitometer.

This facility is accredited to process the following dosineter by virtue of actual demonstration of compliance with ANSI-N13.11-1983 through testing.

Siemens TLD (3 TLD 100, LiF. chips) for ANSI-N13.11 Categories I, II, III, IV, V, VI, VII. Slemens Film Badge (Kodak Type 3, CR-39) for ANSI-N13.11 Categorles III, IV, V, VI, VII, VIII. 


\title{
TELEDYNE ISOTOPES \\ 50 Van Buren Avenue, Westwood, NJ 07675 \\ George Ascione Phone: 201-664-7070
}

Accreditation Renewal Date: October 1, 1987

This facility has been evaluated and deemed competent to process the radiation dosimeters listed below through employing Teledyne Automatic readers model 9100 and 9150 , and Teledyne Manual readers model 8300 and 7300 .

This facility is accredited to process the following dosimeters by virtue of actual demonstration of compliance with ANSI-N13.11-1983 through testing.

Teledyne TLD model PB3 for ANSI-N13.11 categories I, II, III, IV, V, VI, VII, VIII, Teledyne TLD model PB2 for ANSI-N13.1l categories I, II, III, IV, V, VI, VII, VIII.

NVLAP LAB CODE 0534

\author{
QULF STATES UTILITIES - RIVER BEND STATION \\ DOSIMETRY GROUP \\ P.0. Box 220, St. Francisville, LA 70775 \\ Dwight M. Ross Phone: 504-635-6094
}

Accreditation Renewal Date: July 1, 1987

This facility has been evaluated and deemed competent to process the radiation dosimeter listed below through employing a Panasonic Automatic reader model UD710A.

This facility is accredited to process the following dosimeter by virtue of actual demonstration of compliance with ANSI-N13.11-1983 through testing.

Panasonic TLD model 10802 for ANSI-N13.11 categories I, II, III, IV, V, VI, VII, VIII.

\section{NVLAP LAB CODE 0536}

ARIZONA NUCLEAR POWER PROJECT-PVNGS

\section{P.0. Box 21666, Station 6075, Phoenix, AZ 85036}

Michael W. Lantz Phone: 602-932-5300

Accreditation Renewal Date: October 1, 1987

This facility has been evaluated and deemed competent to process the radiation dosimeters listed below through employing a Panasonic Automatic reader model UD710A and Panasonic Manual reader UD720.

This facility is accredited to process the following dosimeter by virtue of actual demonstration of compliance with ANSI-N13.11-1983 through testing.

Panasonic TLD model 10812 for ANSI-N13.11 categories I, II, III, IV, V, VI, VII.

Panasonic TLD combination UD809 and UD812 for ANSI-N13.11 category VIII. 


\section{PACIFIC GAS AND ELECTRIC \\ DIABLO CANYON POWER PLANT \\ Box 337, Avila Beach, CA 93!24 \\ Don Jones Phone: 805-595-7448}

Accreditation Renewal Date: October 1, 1987

This facility has been evaluated and deemed competent to process the radiation dosimeters listed below through employing a Panasonic Automatic reader model UD71OA and Panasonic Manual reader UD702E.

This facility is accredited to process the following dosimeters by virtue of actual demonstration of compliance with ANSI-N13.11-1983 through testing.

Panasonic TLD model 10802 for ANSI-N13.11 categories II, III, IV, V, VI, VII, VIII,

Panasonic TLD model UD813/802 for ANSI-N13.11 category VIII.

NLAP LAB COOE 0538

CON EDISON INDIAN POINT STATION

Broadway and Bleakly Avenue, Buchanan, NY 10511

Philip J. Gaudio Phone: 914-526-5248

Accreditation Renewal Date: April 1, 1988

This facility has been evaluated and deemed competent to process the radiation dosimeter listed below through employing a Panasonic Automatic reader model UD710A and Panasonic Manual reader UD702A.

This facility is accredited to process the following dosimeter by virtue of actual demonstration of compliance with ANSI-N13.11-1983 through testing.

Panasonic TLD model uD802 for ANSI-N13.11 categories II, III, IV, V, VI, VII, VIII.

NVLAP LAB COOE 0539

\section{U.S. ARMY IONIZING RADIATION DOSIMETRY CENTER}

Attn: AMXTM-CE-DC, Lexington, $K Y 40511$

A. Edward Abney Phone: 606-293-3249

Accreditation Renewal Date: January 1, 1988

This facility has been evaluated and deemed competent to process the radiation dosimeters listed below through employing Manual film processing and using a Macbeth model To-504 densitometer.

This facility is accredited to process the following dosimeter by virtue of actual demonstration of compliance with ANSI-N13.11-1983 through testing.

Film Badge (Kodak Type 3) for ANSI-N13.11 Categories I, II, III, IV, V, VI, VII.

Film Badge (Kodak Type A) for ANSI-N13.11 Category VIII. 


\author{
NORTHEAST UTILITIES SERVICE COMPANY \\ RADIOLOGICAL ASSESSMENT BRANCH \\ P.D. Box 270 , Hartford, CT 06141 \\ Henry W. Siegrist Phone: 203-665-3591
}

Accreditation Renewal Date: January 1, 1988

This facility has been evaluated and deemed competent to process the radiation dosimeter listed below through employing Teledyne Automatic reader model 9100, and Teledyne Manual reader model 8300 .

This facility is accredited to process the following dosimeter by virtue of actual demonstration of compliance with ANSI-N13.11-1983 through testing.

Teledyne TLD model PB3 for ANSI-N13.11 categories II, IV, V, VI, VII, VIII.

NVLAP LAB COOE 0541

\author{
COMMONYEALTH EDISON COMPANY \\ 72 West Adams Street, Room 1248 \\ Eileen A. O'Connor Phone: 312-294-8520
}

Accreditation Renewal Date: January 1, 1988

$$
\begin{aligned}
& \text { Dresden Station } \\
& \text { Quad Cities Station } \\
& \text { Zion Station }
\end{aligned}
$$

These facilities listed have been evaluated and deemed competent to process the radiation dosimeter listed below through employing a Panasonic Automatic reader model UD710A.

These facilities are accredited to process the following dosimeter by virtue of actual demonstration of compliance with ANSI-N13.11-1983 through testing.

Panasonic TL model UD802 for ANSI-N13.11 categories I, II, III, IV, V, VI, VII.

\title{
NRLAP LAB COOE 0542
}

\author{
ARIZONA STATE UNIVERSITY \\ RADIATION MEASUREMENTS FACILITV \\ College of Engineering and Applied soiences \\ Electrical and Computer Engineering \\ G. William Klingler \\ 85287
}

Accreditation Renewal Date: October 1, 1988

This facility has been evaluated and deemed competent to process the radiation dosimeter listed below through employing a Panasonic Automatic reader model UD710A.

This facility is accredited to process the following dosimeter by virtue of actual demonstration of compliance with ANSI-N13.11-1983 through testing.

Panasonic ILD model UD812 for ANSI-N13.11 category IV. 


\begin{abstract}
NEW HAMPSHIRE YANKEE
SEABROOK STATION

P.O. Box 300, Route 1, Seabrook, N1 03874

Priscilla J. Neault

Phone: 603-474-9574
\end{abstract}

Accreditation Renewal Date: January 1, 1988

This facility has been evaluated and deemed competent to process the radiation dosimeters listed below through employing a Panasonic Automatic reader model UD71OA and Panasonic Manual reader UD702E.

This facility is accredited to process the following dosimeters by virtue of actual demonstration of compliance with ANSI-N13.11-1983 through testing.

Panasonic TLD model UD813 for ANSI-N13.11 categories I, II, III, IV, V, VI, VII.

Panasonic TLD combination UD809 and UD813 for ANSI-N13.11 category VIII.

NVLAP LAP COOE 0544

FLORIDA POWER \& LIGHT

9250 West Flagler Street

P.0. Box 029110, Mlami, FL 33102

Sander C. Perle Phone: 305-552-3669

Accreditation Renewal Date: July 1, 1988

This facility has been evaluated and deemed competent to process the radiation dosimeter listed below through employing a Harshaw Automatic reader model 8000 .

This facility is accredited to process the following dosimeter by virtue of actual demonstration of compliance with ANSI-N13.11-1983 through testing.

Panasonic TLD model LG-7777 for ANSI-N13.11 categories I, II, III, IV, V, VI, VII and Harshaw ILD model L-NG7677 for ANSI-N13.11 category VIII.

NVAP LAP COOE 0545

Accreditation Renewal Date: July 1, 1988

This facility has been evaluated and deemed competent to process the radiation dosimeters listed below through employing Teledyne Automatic reader model 9100 , and Teledyne Manual reader model 8300 .

This facility is accredited to process the following dosimeters by virtue of actual demonstration of compliance with ANSI-N13.11-1983 through testing.

Teledyne RD model PB3 with CaSO4 Beta/gama card, both primary and back up areas for ANSI-N13-11 category II, IV, V, VII, VIII.

Teledyne TLD model PB3 with CaSO4 Beta/ganma card and 6LIF/CaSO4 Neutron card both primary and back up areas for ANSI-N13-11 category VIII. 


\section{MISSISSIPPI POWER \& LIGHT \\ GRAND GULF NUCLEAR STATION \\ P.0. Box 756, Port Gibson, MS 39150 \\ Tomy E. Tankersley Phone: 601-437-2369}

Accreditation Renewal Date: July 1, 1988

This facility has been evaluated and deemed competent to process the radiation dosimeter listed below through employing a Panasonic Automatic reader model UD71OA and Panasonic Manual reader UD702A.

This facility is accredited to process the following dosimeter by virtue of actual demonstration of compliance with ANSI-N13.11-1983 through testing.

Panasonic TRD model UD802 for ANSI-N13.11 categories I, II, III, IV, V, VI, VII, VIII.

\section{NVLAP LAB CODE 0547}

SOUTH CAROLINA ELECTRIC \& GAS COMPANY

P.0. Box 764, Columbia, SC 29218

Gregory G. Hall Phone: 803-345-1915

Accreditation Renewal Date: July 1, 1988

This facility has been evaluated and deemed competent to process the radiation dosimeter listed below through employing a Panasonic Automatic reader model UD710A.

This facility is accredited to process the following dosimeter by virtue of actual demonstration of compliance with ANSI-N13.11.11-1983 through testing.

Panasonic TLD model UD802 for ANSI-N13.11 categories I, II, III, IV, V, VI VII, VIII. 


\section{NATIONAL VOUUNTARY LABORATORY ACCREDITATION PROGRAM PROCEDURES}

(Title 15, Part 7, of the Code of Federsl Regulations)

(Effective December 1984)

Subpart A - General Information

Sec.

7.1 Purpose.

7.2 Description and goal of NLAP.

7.3 Layout of procedures.

7.4 Definitions.

7.5 Establishment and functions of a National Laboratory

7.6 Accreditation Advisory Committee.

7.7 Information collection Requirements

Subpart B - Establishing a LAP

7.11 Requesting a LAP.

7.12 LAP development decision.

7.13 Request from a government agency.

7.14 Request from a private sector organization.

7.15 Development of tecmical requirements.

7.16 Coordination with federal agencies.

7.17 Announcing the establishment of a LAP.

7.18 Adding to an established LAP.

7.19 Termination of a LAP.

Subpart C - Accrediting a Laboratory

7.21 Applying for accreditation.

7.22 Assessing and evaluating a laboratory.

7.23 Granting and renewing accreditation.

7.24 Denying, suspending, and revoking accreditation.

7.25 Voluntary termination of accreditation

Subpart D - Conditions and Criteria for Accreditation

7.31 Application of accreditation conditions and criteria.

7.32 Conditions for accreditation.

7.33 Criteria for accreditation.

AUTHORITY: Sec. 2, 31 Stat 1449 as amended (15 U.S.C. 272); Reorg. Plan No. 3 of 1946, Part VI.

SUBPART A - GENERAL INFORMATION

Sec. 7.1 Purpose.

The purpose of Part 7 is to set out procedures under which the National Voluntary Laboratory Accreditation Program (NVLAP) will function.

Sec. 7.2 Description and goal of NMAP.

(a) NVLAP is a system for accrediting testing laboratories found competent to perform specific tests or types of tests. Competence is defined as the ability of a laboratory to meet the NVLAP conditions (Section 7.32) and to conform to the criteria (Section 7.33) as tailored and interpreted for the test methods, types of test methods, products, services, or standards for which the laboratory seeks accreditation.

(b) NVLAP is a voluntary system which:

(1) Provides national recognition for competent laboratories;

(2) Provides laboratory management with a quality assurance check of the performance of their laboratories;

(3) Identifies competent laboratories for use by regulatory agencies, purchasing authorities, and product certification systems; and

(4) Provides laboratories with guidance from technical experts to aid them in reaching a higher level of performance resulting in the generation of improved engineering and product information. 
(c) NVLAP is comprised of a series of laboratory accreditation programs (LAPS) which are established on the basis of requests and demonstrated need. The specific test methods, types of test methods, products, services, or standards to be included in a LAP must be requested. The Director of the National Bureau of Standards (NBS) does not unilaterally propose or decide the scope of a LAP. Communication with other laboratory accreditation systems is fostered to encourage development of common criteria and approaches to accreditation and to promote the domestic, foreign, and international acceptance of test data produced by the accredited laboratories.

(d) NVLAP is carried out to be compatible with and recognized by domestic, foreign, and international systems for laboratory accreditation so as to enhance the universal acceptance of test data produced by MLAP-accredited laboratories.

Sec. 7.3 Layout of Procedures.

Subpart $A$ describes considerations which relate in general to all aspects of NMLAP. Subpart $B$ describes how new LAPS are requested, developed and announced, and how LAPs are terminated. Subpart $C$ descrioes procedures for accrediting laboratories. Subpart $D$ sets out the conditions and criteria for NVLAP accreditation.

Sec. 7.4 Definitions.

Accreditation criteria means a set of requirements used by an accrediting body which a laboratory must meet to be accredited.

Advisory Committee means the National Laboratory Accreditation Advisory Committee.

Director of NBS means the Director of the National Bureau of Standards or designee.

Director of OPSP means the Director of the NBS Office of Product Standards Policy or designee.

Laboratory accreditation is a formal recognition that a testing laboratory is competent to carry

aut specific tests or types of tests.

Laboratory assessment means the on-site examination of a testing laboratory to evaluate its

compliance with specified criteria.

LAP means a laboratory accreditation program established and administered under MLAP.

NBS means the National Bureau of Standards.

NVLAP means the National Voluntary Laboratory Accreditation Program.

OPSP means the NBS Office of Product Standards Policy.

Person means associations, companies, corporations, educational institutions, firms, government

agencies at the federal, state and local level, partnerships, and societies-- as well as divisions thereof-and individuals.

Product means a type or a category of manufactured goods, constructions, installations, and

natural and processed materials, or those associated services whose characterization, classification, or functional performance is specified by standards or test methods.

Proficiency testing means methods of checking laboratory testing performance by means of interlaboratory tests.

Testing laboratory is a laboratory which measures, examines, tests, calibrates or otherwise determines the characteristics or performance of products.

Traceability of the accuracy of measuring instruments is a dowisisented chain of comparison connecting the accuracy of a measuring instrument to other measuring instruments of higher accuracy and ultimately to a primary standard.

Sec. 7.5 Establishment and Functions of a National Laboratory Accreditation Advisory Committee.

(a) The Director of NBS shall establish a National Laboratory Accreditation Advisory Committee (Advisory Committee) and appoint its chairperson and members following the filing of a charter setting forth the purpose and nature of the committee.

(b) The composition of the Advisory Comittee will be approximately as follows:

(1) one-third from federal, state and local governments;

(2) One-third from testing laboratories (independent, corporate, and academic); and

(3) One-third from users of testing laboratories, academia, consultants, and consumers.

(c) The Advisory Comittee will be governed by the Federal Advisory Comittee Act (5 U.S.C. App.

2). Persons selected to serve on the Advisory Committee may be paid travel expenses and per diem.

(d) The Advisory Comittee shall function solely in an advisory capacity with functions to include the following:

(1) Assessing the future and continuing role of MLAP and laboratory accreditation in terms of the changing requirements of industry and comerce;

(2) Advising on the technical requirements of testing laboratories and those served by the laboratories;

(3) Advising on the necessity and implementation of proposed amendments to the criteria referenced in Section 7.33;

(4) Evaluating the interaction of other laboratory accreditation systems with NYAP; and 
(5) Reviewing and giving recommendations on the development of international accreditation activities and assessing the impact of such activities on NVLAP.

(e) The Advisory Committee shall meet periodically as called uon by the Director of the NBS office of Product Standards Policy (OPSP) or may be consulted through periodic mailings from the Director of OPSP.

Sec. 7.6 User information.

(a) The Director of OPSP shall prepare and publish at least once each year a directory of accredited laboratories.

(b) The Director of OPSP shall periodically prepare supplements to the directory of accredited laboratories covering new accreditation actions taken, including initial accreditations, renewals, suspensions, terminations, and revocations.

Sec. 7.7 Information Collection Requirements.

The information collection requirements contained in these procedures have been approved by the Office of Management and Budget under the Paperwork Reduction Act and have been assigned OMB control number 0652-0003.

SUBPART B - ESTABLISHING A LAP

Sec. 7.11 Requesting a LAP.

(a) Any person may request the Director of NBS to establish a LAP.

(b) Each request must be in writing and must include:

(1) The scope of the LAP in terms of products or testing services proposed for inclusion;

(2) Specific identification of the applicable standards and test methods including appropriate designations, and the organizations or standards writing bodies having responsibility for them;

(3) A statement of need for the LAP including:

(i) Technical and economic reasons why the LAP would benefit the public interest;

(ii) Evidence of a national need to accredit testing laboratories for the specific scope beyond that served by an existing laboratory accreditation program in the public or private sector;

(iii) An estimate of the number of laboratories that may seek accreditation; and

(iv) An estimate of the number and nature of the users of such laboratories; and

(4) A statement of the extent to wich the requestor is willing to support necessary developmental aspects of the LAP with funding and personnel.

(c) The Director of OPSP may request clarification of the information required by paragraph (b) of this section.

(d) Before determining the need for a LAP, the Director of NBS shall publish a FEDERAL REGISTER notice of the receipt of a LAP request if the request complies with section 7.11(b). The not ice will:

(i) Describe the scope of the requested LAP;

(2) Indicate how to obtain a copy of the request; and

(3) State that anyone may submit corments on the need for a LAP to the Director of OPSP within 60 days of the date of the notice.

Sec. 7.12 LAP development decision.

(a) The Director of NBS shall establish all LAPS on the basis of need. Government agencies and private sector organizations may establish the need by using sections 7.13 and 7.14.

(b) After receipt of the request, the Director of NBS shall analyze it to determine if a need exists for the requested LAP. In making this determination, the Director of NBS shall consider the following:

(1) The needs and scope of the LAP initially requested;

(2) The needs and scope of the user population;

(3) The nature and content of other relevant public and private sector laboratory accreditation programs;

(4) Compatibility with the criteria referenced in section 7.33 ;

(5) The importance of the requested LAP to comerce, consumer well-being, or the public health and safety;

(6) The economic and technical feasibility of accrediting testing laboratories for the test methods, types of test methods, products, services, or standards requested; and

(7) Recommendations from written coments for altering the scope of the requested LAP by adding or deleting test methods, types of test methods, products, services, or standards. 
(c) If the Director of NBS decides that a need has been demonstrated, and if resources are available to develop a LAP, the Director of OPSP shall notify interested persons of the decision to proceed with development of a LAP.

(d) If the Director of NBS concludes that there is a need for a LAP but there are no resources for development, the Director of OPSP shall notify the requestor and other interested persons of the decision not to proceed until resources become available.

(e) If the Director of NBS decides that a need for a LAP has not been demonstrated, the Director of OPSP shall notify the requestor and other interested persons of the decision and the reasons not to proceed with development of a LAP.

Sec. 7.13 Request from a government agency.

(a) Any federal, state or local agency responsible for regulatory or public service programs established under statute or code, wich has determined a need to accredit testing laboratories within the context of its programs, may request the Director of NBS to establish a LAP.

(b) Each request must be in writing and must include the information required in section 7.11 (b) and:

(1) A description of the procedures followed or a citation of the specific authority used to determine the need for a LAP; and

(2) For state and local government agencies, a statement of why the LAP should be of national scope.

(c) The Director of OPSP may request clarification of the information required by paragraph (b) of this section.

(d) Before deciding to proceed with development of a LAP, the Director of NBS shall publish a FEDERAL REGISTER notice of the receipt of a LAP request. The notice will indicate how to obtain a copy of the request and will state that anyone may submit comments on the need for a LAP to the requesting government agency within 60 days of the date of the notice.

(e) The Director of OPSP shall notify interested persons of the decision to proceed or not to proceed with development of a LAP.

Sec. 7.14 Request from a private sector organization.

(a) Any private sector organization which has determined a need to accredit testing laboratories for specific products or testing services, may request the Director of NBS to establish a LAP if it uses procedures meeting the following conditions:

(1) Public notice of meetings and other activities including requests for LAPs is provided in a timely fashion and is distributed to reach the attention of interested persons;

(2) Meetings are open and participation in activities is available to interested persons;

(3) Decisions reached by the private sector organization in the development of a request for a LAP represent substantial agreement of the interested persions;

(4) Prompt consideration is given to the expressed views ano concerns of interested persons;

(5) Adequate and impartial mechanisms for handling substantive and procedural complaints and appeals are in place; and

(6) Appropriate records of all meetings are maintained and the official procedures used by the private sector organization to make a formal request for a LAP are made available upon request to any interested person.

(b) Each request must be in writing and must include the information required in Section 7.11 (b) and a description of the way in which the organization has met the conditions specified in paragraph (a) of this section.

(c) The Director of OPSP may request clarification of the information required by paragraph (b) of this section.

(d) Before deciding to proceed with development of a LAP, the Director of NBS shall publish a FEDERAL REGISTER notice of the receipt of a LAP request. The notice will indicate how to obtain a copy of the request and will state that anyone may submit comments on the need for a LAP to the requesting private sector organization within 60 days of the date of the notice.

(e) The Director of OPSP shall notify interested persons of the decision to proceed or not to proceed with development of a LAP. 
Sec. 7.15 Development of technical requirements.

(a) Technical requirements for accreditation are specific for each LAP. The requirements tailor the criteria referenced in section 7.33 to the test methods, types of test methods, products, services, or standards covered by the LAP.

(b) The Director of OPSP shall develop the technical requirements based on expert advice. This advice may be obtained through one or more informal public workshops or other suitable means.

(c) The Director of OPSP shall make every reasonable effort to ensure that the affected testing community within the scope of the LAP is informed of any planned workshop. Summary minutes of each workshop will be prepared. A copy of the minutes will be made available for inspection and copying at the NBS Records Inspection Facility.

\section{Sec. 7.16 Coordination with federal agencies.}

As a means of assuring effective and meaning ful cooperation, input, and participation by those federal agencies that may have an interest in and may be affected by established LAPs, the Director of OPSP shall communicate and consult with appropriate officials within those agencies.

Sec. 7.17 Announcing the establishment of a LAP.

(a) When the Director of OPSP has completed the development of the technical requirements of the LAP and established a schedule of fees for accreditation, the Director of OPSP shall publish a notice in the FEDERAL REGISTER announcing the establishment of the LAP.

(b) The notice will:

(1) Identify the scope of the LAP; and

(2) Advise how to apply for accreditation.

(c) The Director of OPSP shall establish fees in amounts that will enable the LAP to be self-sufficient. The Director of OPSP shall revise the fees when necessary to maintain self-sufficiency.

Sec. 7.18 Adding to an established LAP.

Written requests will be considered from any person wishing to add specific standards, test methods, or types of test methods to an established or developing LAP. The Director of OPSP may choose to make them available for accreditation under a LAP when:

(a) The additional standards, test methods, or types of test methods requested are directly relevant to the LAP;

(b) It is feasible and practical to accredit testing laboratories for the additional standards, test methods, or types of test methods; and

(c) It is likely that laboratories will seek accreditation for the additional standards, test methods, or types of test methods.

Sec. 7.19 Termination of a LAP.

(a) The Director of NBS may terminate a LAP when the Director of NBS determines that a need no longer exists to accredit testing laboratories for the products or testing services covered under the scope of the LAP. In the event that the Director of NBS proposes to terminate a LAP, a notice will be published in the FEDERAL REGISTER setting forth the basis for that determination.

(b) The notice published under paragraph (a) of this section will provide a 60-day period for submitting written comments on the proposal to terminate the LAP. All written comments will be made avallable for public inspection and copying in the NBS Records Inspection Facility.

(c) After the comment period, the Director of NBS shall determine if public support exists for the continuation of the LAP. If public corments support the continuation of the LAP, the Director of NBS shall publish a FEDERAL REGISTER notice announcing the continuation of the LAP. If public support does not exist for continuation, the LAP will be terminated effective 90 days after the date of the published notice of intent to terminate the LAP.

(d) If the LAP is terminated, the Director of OPSP shall no longer grant or renew accreditations following the effective date of termination. Accreditations previously granted will remain effective until their expiration date unless terminated voluntarily by the laboratory or revoked by the Director of OPSP. 
Sec. 7.21 Applying for accreditation.

(a) Any laboratory may request an application for accreditation in any established LAPs in accordance with instructions provided in notices announcing the formal establishment of LAPs.

(b) Upon receipt of a laboratory's application, the Director of OPSP shall:

(1) Acknowledge receipt of the application;

(2) Request further information, if necessary;

(3) Confirm payment of fees before proceeding with the accreditation process; and

(4) Specify the next $\operatorname{step}(s)$ in the accreditation process.

(c) In accepting an application from a foreign-based laboratory, the Director of OPSP shall take into consideration the policy of the host government regarding the acceptance of test data from laboratories accredited by NVLAP or other foreign accreditation systems.

Sec. 7.22 Assessing and Evaluating a laboratory.

(a) Information used to evaluate a laboratory's compliance with the conditions for accreditation set out in section 7.32, the criteria for accreditation set out in Section 7.33, and the technical requirements established for each LAP will include:

(1) On-site assessment reports;

(2) Laboratory responses to identified deficiencies; and

(3) Laboratory performance on proficiency tests.

(b) The Director of OPSP shall arrange the assessment and evaluation of applicant laboratories by contract or other means in such a way as to minimize potential conflicts of interest.

(c) The Director of OPSP shall inform each applicant laboratory of any action(s) that the laboratory must take to complete the requirements for assessment and evaluation.

Sec. 7.23 Granting and renewing accreditation.

(a) The Director of OPSP, after reviewing an evaluation report, shall grant or renew, suspend, or propose to deny or revoke accreditation of an applicant laboratory, no later than 30 days following the date of submittal of the report. If accreditation action is not taken within this time limit, the Director of OPSP shall notify the laboratory stating the reasons for the delay.

(b) If accreditation is granted or renewed, the Director of OPSP shall:

(1) Provide a certificate of accreditation to the laboratory;

(2) Identify the scope and terms of the laboratory's accreditation;

(3) Provide guidance on referencing the laboratory's accredited status, and the use of the NVLAP logo by the laboratory and its clients, as needed; and

(4) Remind the laboratory that accreditation does not relieve it from complying with applicable federal, state, and local laws and regulations.

(c) The Director of OPSP shall notify an accredited laboratory at least 30 days before its accreditation expires advising of the action(s) the laboratory must take to renew its accred itation.

(d) If an accredited laboratory fails to complete the assessment and evaluation process for renewal before its accreditation expires, the Director of OPSP shall notify the laboratory stating that its accreditation has expired and reiterating the action(s) the laboratory must take to renew its accreditation.

Sec. 7.24 Denying, suspending, and revoking accreditation.

(a) If the Director of OPSP proposes to deny or revoke accreditation of a laboratory, the Director of OPSP shall inform the laboratory of the reasons for the proposed denial or revocation and the procedure for appealing such a decision.

(b) The laboratory will have 30 days from the date of receipt of the proposed denial or revocation letter to request a hearing under the provisions of 5 U.S.C. 556. If the laboratory requests a hearing, the proposed denial or revocation will be stayed pending the outcome of the hearing held under provisions of 5 U.S.C. 556. The proposed denial or revocation will become final through the issuance of a written decision to the laboratory in the event that the laboratory does not appeal the proposed denial or revocation within that 30-day period.

(c) If the Director of OPSP pinds that an accredited laboratory has violated the terms of its accreditation or the provisions of these procedures, the Director of OPSP may, after consultation with the laboratory, suspend the laboratory's accreditation, or advise of his/her intent to revoke its accreditation. If accreditation is suspended, the Director of OPSP shall notify the laboratory 
of that action stating the reasons for and conditions of the suspension and specifying the action(s) the laboratory must take to have its accreditation reinstated. Conditions of suspension will include prohibiting the laboratory from using the NRLAP logo on its test reports during the suspension period. The determination of the Director of OPSP whether to suspend or to propose revocation of a laboratory's accreditation will depend on the nature of the violation(s) of the terms of its accreditation.

(d) A laboratory whose accreditation has been denied, revoked, terminated, or expired, or which has withdrawn its application before being accredited, may reapply and be accredited if the laboratory:

(1) Completes the assessment and evaluation process; and

(2) Meets the conditions and criteria for accreditation that are set out in Subpart D;

Sec. 7.25 Voluntary termination of accreditation.

A laboratory may at any time terminate its participation and responsibilities as an accredited laboratory by advising the Director of OPSP in writing of its desire to do so. The Director of OPSP shall terminate the laboratory's accreditation and shall notify the laboratory stating that its accreditation has been terminated in response to its request.

\section{SUBPART D - CONDITIONS AND CRITERIA FOR ACCREDITATION}

Sec. 7.31 Application of accreditation conditions and criteria.

(a) To become accredited and maintain accreditation, a laboratory must meet the conditions for accreditation set out in section 7.32 and the criteria set out in Section 7.33 as tailored for specific LAPs.

(b) The conditions leading to accreditation include acceptance of the responsibilities of an accredited laboratory and requirements for information disclosure.

(c) The criteria are tailored and interpreted for the test methods, types of test methods, products, services or standards of the relevant LAP. These tailored Criteria are the technical requirements for accreditation developed through the procedures of section 7.15.

(d) In applying the conditions, criteria, and technical requirements for accreditation, the Director of OPSP shall not:

(1) Prohibit accreditation solely on the basis of a laboratory's affiliation or nonaffiliation with manufacturing, distributing, or vending organizations, or because the laboratory is a foreign firm; or

(2) Develop, modify, or promulgate test methods, standards, or comparable administrative rules.

Sec. 7.32 Conditions for accreditation.

(a) To become accredited and maintain accreditation, a laboratory shall agree in writing to:

(1) Be assessed and evaluated initially and on a periodic basis;

(2) Demonstrate, on request, that it is able to perform the tests representative of those for which it is seeking accreditation;

(3) Pay all relevant fees:

(4) Participate in proficlency testing as required.

(5) Be capable of performing the tests for which it is accredited according to the latest version of the test method within one year after its publication or within another time Iimit specified by the Director of OPSP;

(6) Limit the representation of the scope of its accreditation to only those tests or services for which accreditation is granted;

(7) Limit all its test work or services for clients to those areas where competence and capacity are available;

(8) Limit advertising of its accredited status to letterheads, brochures, test reports, and professional, technical, trade, or other laboratory services publications, and use the NMLAP logo under guidance provided by the Director of OPSP:

(9) Inform its clients that the laboratory's accreditation or any of its test reports in no way constitutes or implies product certification, approval, or endorsement by NBS;

(10) Maintain records of all actions taken in response to testing complaints for a minimum of one year;

(11) Maintaín an independent decisional relationship between itself and its clients, affiliates, or other organizations so that the laboratory's capacity to render test reports objectively and without bias is not adversely affected;

(12) Report to the Director of OPSP within 30 days any major changes involving the location, ownership, management structure, authorized representative, approved signatories, or facilities of the laboratory; and

(13) Return to the Director of OPSP the certificate of accreditation for possible revision or other action should it:

(i) be requested to do so by the Director of OPSP;

(ii) voluntarily terminate its accredited status; or 
(iii) become unable to conform to any of these conditions or the applicable criteria of Section 7.33 and related techrical requirements.

(b) To become accredited and maintain accreditation, a laboratory shall supply, upon request, the

following information:

(1) Legal name and full address;

(2) Ownership of the laboratory;

(3) Organization chart defining relationships that are relevant to performing testing covered in the accreditation request;

(4) Ceneral description of the laboratory, including its facilities and scope of operation;

(5) Name and telephone number of the authorized representative of the laboratory;

(6) Names or titles and qualifications of laboratory staff nominated to serve as approved signatories of test reports that reference NVLAP accreditation; and

(7) Other information as may be needed for the specific LAP(s) in which accreditation is sought.

Sec. 7.33 Criteria for accreditation.

(a) Quality System. (1) The laboratory shall operate under an intemal quality assurance program appropriate to the type, range, and volume of work performed. The quality assurance program must be designed to ensure the required degree of accuracy and precision of the laboratory's work and should include key elements of document control, sample control, data validation, and corrective action. The quality assurance program must be documented in a quality manual or equivalent (e.g., operations notebook) which is available for use by laboratory staff. A person(s) must be identified as having responsibility for maintaining the quality manual.

(2) The quality manual must include as appropriate:

(i) The laboratory's quality assurance polfcies including procedures for corrective action for detected test discrepancies;

(ii) Quality assurance responsibilities for each function of the laboratory;

(iii) Specific quality assurance practices and procedures for each test, type of test, or other specifically delineated function performed;

(iv) Specific procedures for retesting, control charts, reference materials, and interlaboratory tests; and

(v) Procedures for dealing with testing complaints.

(3) The laboratory shall periodically review its quality assurance system by or on behalf of management to ensure it's continued effectiveness. These reviews must be recorded with details of any corrective action taken.

(b) Staff. (1) The laboratory shall:

(1) Be staffed by individuals having the necessary education, training, technical knowledge, and experience for their assigned functions; and

(ii) Have a job description for each professional, scientific, supervisory and technical position, including the necessary education, training, technical knowledge, and experience.

(2) The laboratory shall document the test methods each staff' member has been assigned to perform.

(3) The laboratory shall have a description of its training program for ensuring that new or untrained staff are able to perform tests properly and uniformly to the requisite degree of precision and accuracy.

(4) The laboratory shall be organized:

(i) So that staff members are not subjected to undue pressure or inducement that might influence their judgment or results of their work; and

(ii) In such a way that staff members are aware of both the extent and the limitation of their area of responsibility.

(5) The laboratory shall have a technical manager (or similar title) who has overall responsibility for the techical operations of the laboratory.

(6) The laboratory shall have one or more signatories approved by the Director of OPSP to sign test reports that reference NVLAP accreditation. Approved signatories shall:

(i) Be competent to make a critical evaluation of test results; and

(ii) Occupy positions within the laboratory's organization which makes them responsible for the adequacy of test results.

(c) Facilities and Equipment. (1) The laboratory shall be fumished with all items of equipment and facilities for the correct performance of the tests and measurements for which accreditation is granted and shall have adequate space, lighting, and environmental control, and monitoring to ensure compliance with prescribed testing conditions.

(2) All equipment must be properly maintained to ensure protection from corrosion and other causes of deterioration. Instructions for a proper maintenance procedure for those items of equipment which require periodic maintenance must be available. Any item of equipment or component thereof which has been subjected to over loading or mishandling, gives suspect results, or has been shown by calibration or otherwise to be de fective, must be taken out of seivice and clearly labelled until it has been repaired. When placed back in service, this aquipment must be rhown by test or calibration to be performing its function satisfactorily.

(3) Recurds of each ma jor item of equipment must be maintained. Each record must include:

(i) The name of the item of equipment; 
(ii) The manufacturer's name and type, identification and serial number;

(iii) Date received and date placed in service;

(iv) Current location, where appropriate;

(v) Details of maintenance; and

(vi) Date of last calibration, next calibration due date, and calibration report references.

(d) Calibration. The laboratory shall.

(I) Callorate new testing equipment before putting it into service;

(2) Recalibrate, at regular intervals, in-service testing equipment with the calibration status readily available to the operator;

(3) Perform checks of in-service testing equipment between the regular calibration intervals, where relevant;

(4) Maintain adequate records of all calibrations and recalibrations; and

(5) Provide traceability of all calibrations and reference standards of measurement where these standards exist. Where traceability of measurements to primary (national or international) standards is not applicable, the laboratory shall provide satisfactory evidence of the accuracy or reliability of test results (e.g., by participation in a suitable program of inter laboratory comparison).

(e) Test Methods and Procedures. The laboratory shall:

(1) Conform in all respects with the test methods and procedures required by the specifications against wich the test item is to be tested, except that whenever a departure becomes necessary for techical reasons the departure must be acceptable to the client and recorded in the test report;

(2) Have data to prove that any departures from standard methods and/or procedures due to apparatus design or for other reasons do not detract from the expected or required precision of the measurement;

(3) Maintain a test plan for implementing testing standards and procedures including adequate instructions on the use and operation of all relevant equipment, on the handling and preparation of test items (where applicable), and on standard testing techniques where the absence of such instructions could compromise the test. All instructions, testing standards, specifications, manuals, and reference data relevant to the work of the laboratory must be kept up-to-date and made readily available to the staff;

(4) Maintain measures for the detection and resolution of in-process testing discrepancies for manual and automatic test equipment and electronic data processing equipment, where applícable;

(5) Maintain a system for identifying samples or items to be tested, which remains in force from the date of receipt of the item to the date of its disposal, either through documents or through marking to ensure that there is no confusion regarding the identity of the samples or test items and the results of the measurements made; and

(6) Maintain rules for the receipt, retention, and disposal of test items, including procedures for storage and handling precautions to prevent damage to test items which could invalidate the test results. Any relevant instructions provided with the tested item must be observed.

(f) Records. The laboratory shall:

(I) Maintain a record system which contains sufficient information to permit verification of any issued report;

(2) Retain all original observations, calculations and derived data, and calibration records for one year unless a longer period is specified; and

(3) Hold records secure and in confidence, as required.

(g) Test Reports. (1) The laboratory shall issue test reports of its work which accurately, clearly, and unambiguously present the specified test results and all required information. Each test report must include the following information as applicable:

(i) Name and address of the laboratory;

(ii) Identification of the test report by serial number, date, or other appropriate means;

(iii) ficme and address of client;

(iv) Description and identification of the test specimen, sample, or lot of material represented;

(v) Identification of the test specification, method, or procedure used;

(vi) Description of sampling procedure, if appropriate;

(vii) Any deviations, additions to, or exclusions from the test specifications;

(viii) Measurements, examinations, and derived results supported by tables, graphs, sketches, and photographs, as appropriate, and any failures identified;

(ix) A statement of measurement uncertainty where relevant;

(x) Identification of the organization and the person accepting technical responsibility for the test report and date of issue;

(xi) A statement that the report must not be reproduced except in full with the approval of the laboratory; and

(xii) A statement to the effect that the test report relates only to the items tested. 
(2) The laboratory shall issue corrections or additions to a test report only by a further document suitably marked, e.g. "Supplement to test report serial number .....," which meets the relevant requirements of section $7.33(\mathrm{~g})(1)$.

(3) The laboratory shall retain a copy of each test report issued for one year unless a longer period is specified by the Director of OPSP.

(4) The laboratory shall ensure that all test reports endorsed with the NVLAP logo are signed by an approved signatory. 
BIBLIOGRAPHIC DATA

SHEET (See in structions)

4. TITLE AND SUBTITLE

\section{6-87 Directory of NVLAP Accredited Laboratories}

5. $A U T H O R(S)$

Harvey W. Berger

6. PERFORMING ORGANIZATION (If joint or other than NBS, see instructlons)

NATIONAL BUREAU OF STANDARDS

DEPARTMENT OF COMMERCE

WASHINGTON, D.C. 20234

9. SPONSORING ORGANIZATION NAME AND COMPLETE ADDRESS (Street, City, State, ZIP)

7. Contract/Grant No.

Same as 6.

10. SUPPLEMENTARY NOTES

Document describes a computer program; SF-185, FIPS Software Summary, is attached.

11. ABSTRACT (A 200-word or less factual summary of most significant information. If document includes a slgnificant bibliography or literature survey. mention it here)

This 1986-87 Directory of NVLAP Accredited Laboratories lists laboratories accredited under the procedures of the National Voluntary Laboratory Accreditation Program (NVLAP) as of January 1, 1987. Indexes cross reference the laboratories by name, NVLAP Lab Code Number, test method, accreditation program, and geographical location. The scope of accreditation of each laboratory, listing the test methods for which it is accredited, is provided along with a tabulation of test methods and the laboratories accredited for those test methods.

12. KE electromagnetic compatibility, laboratory, paint, paper, sealants, seals, stoves, telecommunications, thermal insulation

\section{AVAILABILITY}

- Unlimited

$\square$ For Official Distribution. Do Not Release to NTIS

$\square$ Order From Superintendent of Documents, U.S. Government Printing Office, Washington, D.C. 20402.

[ Order From National Technical Information Service (NTIS), Springfield, VA. 2216I
14. NO. OF PRINTED PAGES

15. Price 

